Design, synthesis and biological evaluation of novel DNA gyrase Inhibitors and their siderophore mimic conjugates

\title{
pÿLamut, Andra
}

2020-01

pÿLamut , A , Cruz , C D , Skok , \}, Baranoková , M , Zidar , N , Zega , A, Peterlin Maai , L pÿ, llaa , J , Tammela , P , Kikelj , D \& Tomaai , T 2020 , ' Design, synthesis and biological evaluation of novel DNA gyrase Inhibitors and their siderophore mimic conjugates ' , Bioorganic Chemistry , vol. 95 , 103550 . https://doi.org/10.1016/j.bioorg.2019.103550

http://hdl.handle.net/10138/337992

https://doi.org/10.1016/j.bioorg.2019.103550

cc_by_nc_nd

acceptedVersion

Downloaded from Helda, University of Helsinki institutional repository.

This is an electronic reprint of the original article.

This reprint may differ from the original in pagination and typographic detail.

Please cite the original version. 
Design, Synthesis and Biological Evaluation of Novel DNA Gyrase Inhibitors and their Siderophore Mimic Conjugates

Andraž Lamut, ${ }^{\mathrm{a}}$ Cristina D. Cruz, ${ }^{\mathrm{b}}$ Žiga Skok, ${ }^{\mathrm{a}}$ Michaela Barančoková, ${ }^{\mathrm{a}}$ Nace Zidar, ${ }^{\mathrm{a}}$ Anamarija Zega, ${ }^{a}$ Lucija Peterlin Mašič, ${ }^{a}$ Janez Ilaš, ${ }^{a}$ Päivi Tammela, ${ }^{b}$ Danijel Kikelj, ${ }^{a}$ and Tihomir Tomašič ${ }^{\text {a,* }}$

${ }^{a}$ University of Ljubljana, Faculty of Pharmacy, Aškerčeva cesta 7, 1000 Ljubljana, Slovenia

${ }^{b}$ Drug Research Program, Division of Pharmaceutical Biosciences, Faculty of Pharmacy, University of Helsinki, P.O. Box 56 (Viikinkaari 5 E), FI-00014 Helsinki, Finland

*Corresponding author: Tihomir Tomašič

University of Ljubljana, Faculty of Pharmacy,

Aškerčeva cesta 7, 1000 Ljubljana, Slovenia

Tel: $+386-1-4769556$

E-mail: tihomir.tomasic@ffa.uni-lj.si 


\title{
Abstract
}

Bacterial DNA gyrase is an important target for the development of novel antibacterial drugs, which are urgently needed because of high level of antibiotic resistance worldwide. We designed and synthesized new 4,5,6,7-tetrahydrobenzo[ $[d]$ thiazole-based DNA gyrase B inhibitors and their conjugates with siderophore mimics, which were introduced to increase the uptake of inhibitors into the bacterial cytoplasm. The most potent conjugate $\mathbf{3 4}$ had an $\mathrm{IC}_{50}$ of $58 \mathrm{nM}$ against Escherichia coli DNA gyrase and displayed MIC of $14 \mu \mathrm{g} / \mathrm{mL}$ against $E$. coli $\Delta$ tolC strain. Only minor improvements in the antibacterial activities against wild-type $E$. coli in low-iron conditions were seen for DNA gyrase inhibitor - siderophore mimic conjugates.

\section{Graphical abstract}

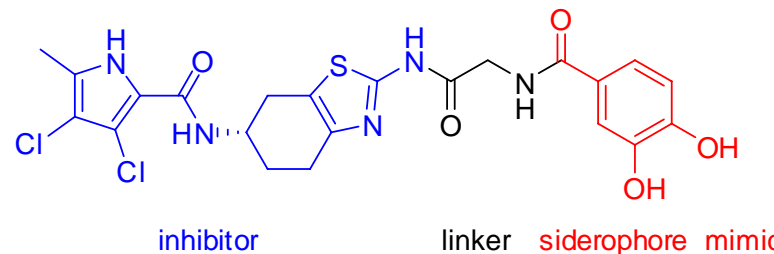

E. coli DNA gyrase $\mathrm{IC}_{50}=58 \mathrm{nM}$

E. coli JW5503 MIC $=14 \mu \mathrm{g} / \mathrm{mL}$

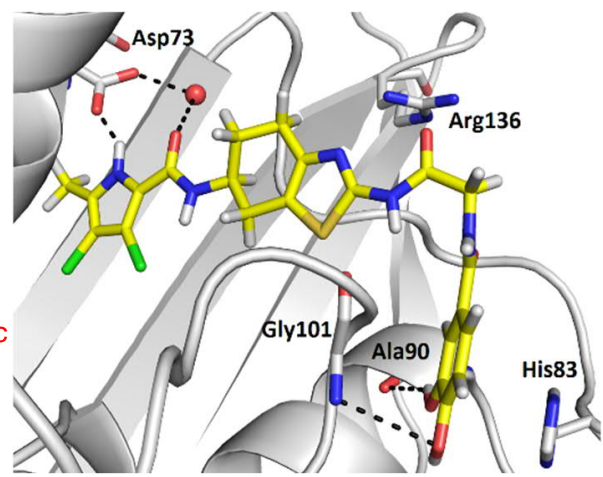

Keywords: antibiotics; catechol; DNA gyrase; inhibitors; siderophore mimic;

\begin{abstract}
Abbreviations
EDC, 1-ethyl-3-(3-(dimethylamino)propyl)-carbodiimide; GyrB, DNA gyrase subunit B; HOBt, 1-hydroxybenzotriazole; MIC, minimum inhibitory concentration; OM, outer membrane.
\end{abstract}




\section{Highlights}

- Siderophore mimics were conjugated with 4,5,6,7-tetrahydrobenzo[d]thiazole-based DNA gyrase B inhibitors.

- The most potent conjugate had an $\mathrm{IC}_{50}$ of $58 \mathrm{nM}$ against Escherichia coli DNA gyrase.

- Two conjugates displayed MICs of $14 \mu \mathrm{g} / \mathrm{mL}$ against $E$. coli $\Delta$ tolC strain.

- Most conjugates showed improved activities against wild-type Escherichia coli in irondepleted medium. 


\section{Introduction}

In recent decades, infectious diseases have been recognized as one of the most important global health issues, which is mainly attributed to the increasing problem of microbial resistance [1, 2]. According to the World Health Organization, the priority pathogens for which research and development of new treatment options are most urgently needed include 12 families of bacteria. Among these, Staphylococcus aureus, Pseudomonas aeruginosa and the Enterobacteriaceae family (including Escherichia coli) have been described as critical high-priority pathogens that are especially difficult to treat [3].

Unfortunately, the urgent need for development of new antibiotics is, however, accompanied by a lack of interest of the pharmaceutical industry for economic reasons and because of strict regulatory requirements [4]. Due to the problem of bacterial resistance, antibiotics with new mechanisms of action are needed, as they would then not be expected to be removed by the pre-existing resistance mechanisms, with the development of cross resistance also less likely to occur. In the last 10 years, some successful new drugs have been approved that have alternative mechanisms of action; e.g., antibiotics belonging to the class of oxazolidinones and lipopeptides [5]. In addition to overexpression of efflux pumps, reduced permeability of the outer membrane $(\mathrm{OM})$ is recognized as a major mechanism that underlies resistance of Gram-negative bacteria and that can cause the failure of existing antibacterial therapies [6].

DNA gyrase is a validated target for antibacterial drug discovery [7,8]. This enzyme belongs to the class of bacterial type IIa topoisomerases, and it is responsible for the introduction of negative supercoils during bacterial DNA replication. DNA gyrase is a heterotetrameric protein that is composed of two GyrA subunits and two ATP-binding GyrB subunits [7]. Many studies have already been carried out to develop clinically useful ATP- 
competitive GyrB inhibitors; however, none are currently available for therapeutic use $[8,9]$. In 2015 we discovered structurally novel GyrB inhibitors based on the 4,5,6,7tetrahydrobenzo[ $[d]$ thiazole-2,6-diamine core (1-3, Figure 1) [10,11]. Compounds 1 and 2 showed low-nanomolar inhibition of $E$. coli DNA gyrase, but did not show activity against wild-type Gram-negative strains of $E$. coli and $P$. aeruginosa, which was most likely the consequence of cell-wall penetration issues and/or active efflux from the bacterial cells that is mediated by their efflux pump machineries. This latter explanation was supported by the findings that some of these compounds showed improved antibacterial activities when tested against the E. coli JW5503 strain that has a defective efflux pump, with MICs as low as $31 \mu \mathrm{M}$ (e.g. 2, Figure 1) [10]. Similar observations were also made for other structural classes of GyrB inhibitors, including benzo[d]thiazoles [12], $N$-phenylpyrrolamides [13] and ethyl ureas [14, 15].

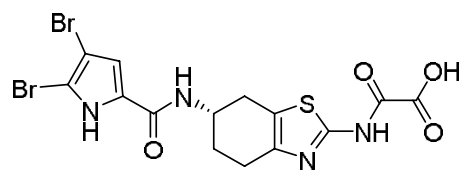

1

E. coligyrase $\mathrm{IC}_{50}: 0.058 \mu \mathrm{M}$ E. coli JW5503 MIC: $520 \mu \mathrm{M}$

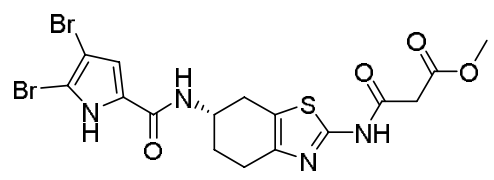

2

E. coligyrase $\mathrm{IC}_{50}: 0.096 \mu \mathrm{M}$ E. coli JW5503MIC: $31 \mu \mathrm{M}$

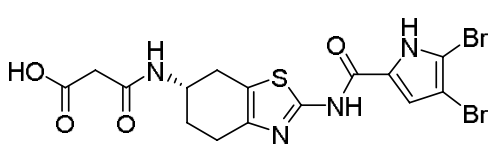

3

E. coligyrase $\mathrm{IC}_{50}: 0.891 \mu \mathrm{M}$

Figure 1. Representative structures of previously reported DNA gyrase inhibitors with the 4,5,6,7tetrahydrobenzo[ $[d]$ thiazole-2,6-diamine scaffold.

One strategy to overcome the problem of low bacterial cell-wall penetration is conjugation of small molecular weight siderophore mimics to molecules that have antibacterial properties $[16,17]$. Siderophores are high-affinity iron-chelating compounds that are secreted by microorganisms and are among the strongest known soluble iron-binding agents [18]. Bacteria use siderophores to exploit the very limited amounts of freely available iron in the host environment during infection, and hence to survive the proliferation process [19]. Ironsiderophore complexes are then transported across the bacterial membrane into the cytoplasm 
via iron-uptake machineries. Attaching a siderophore or a siderophore mimic to an antibacterial drug can therefore be used as a means to deliver antibiotics into bacteria [20, 21]. Siderophore mimics are small fragments of natural siderophores that have the similar structural features that are responsible for iron chelation. Commonly used siderophore mimics are shown in Figure 2, and these include catechols, hydroxypyranones, hydroxypyridones and dihydroxypyridones. Some antibiotic-siderophore mimic conjugates have recently been taken to clinical trials, such as the monosulfactam BAL30072 [22] and cephalosporin cefiderocol [23] (Figure 2). In November 2019 U.S. Food and Drug Administration approved cefiderocol for the treatment of complicated urinary tract infections [24].

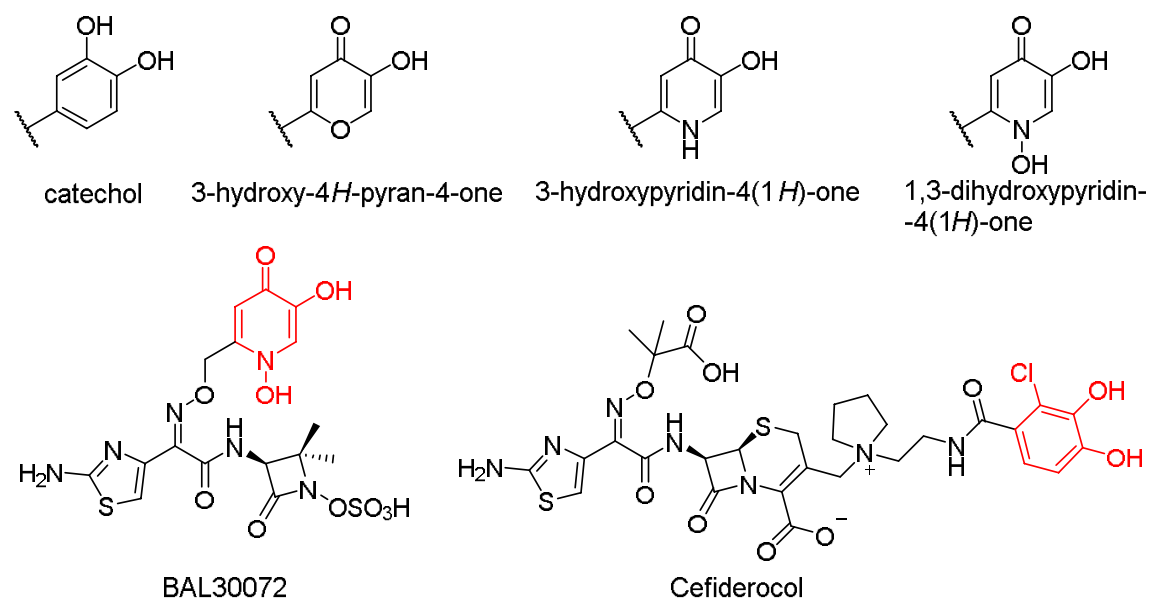

Figure 2. Structures of the commonly used siderophore mimics and the most developed antibiotic-siderophore mimic conjugates BAL30072 and cefiderocol.

In the present study, we designed new DNA gyrase inhibitors starting from compounds presented in Figure 1. Moreover, we attached the siderophore mimics to selected DNA gyrase inhibitors to evaluate their impact on enzyme inhibition and antibacterial activities against Gram-negative bacteria. 


\section{Design}

New (S)-4,5,6,7-tetrahydrobenzo[d]thiazol-6-yl-1H-pyrrole-2-carboxamide derivatives were designed using co-crystal structures that we reported previously, where the inhibitors bind to the 43-kDa N-terminal fragment of E. coli GyrB (PDB codes: 4ZVI, 5L3J) [11, 25]. In these structures, the pyrrole-2-carboxamide moiety is bound deep in the hydrophobic pocket and forms hydrogen bonds with Asp73 of E. coli GyrB and a conserved water molecule. In addition, substituents on the 2-amino group of the central $(S)-4,5,6,7$-tetrahydrobenzo[ $[d]$ thiazole-2,6diamine scaffold can form additional interactions with Arg136 and adjacent amino acid residues of $E$. coli GyrB, as was predicted by molecular docking (Figure 3A) [10].

In the present designed series of DNA gyrase inhibitors and inhibitor-siderophore mimic conjugates, two different pyrrole moieties were used to achieve favorable interactions in the hydrophobic pocket of $E$. coli GyrB, namely dibromopyrrole and dichloromethylpyrrole, as halogen atoms have been shown to be important for binding $[10,26]$. Further, based on the described orientation of these inhibitors in the GyrB ATP-binding site, the 2-amino group was identified as a suitable position on which to attach additional substituents or siderophore mimic moieties (Figure 3).

Based on molecular docking calculations (Figure 3A), substituents directly attached to the 2-amino group (Figure 3B and 3C, type I) can form additional interactions with the binding site residues. In addition, to explore the impact of siderophore mimics on DNA gyrase inhibition, we also designed analogs that had the siderophore mimic attached via different linkers to the 2-amino group of the central scaffold (Figure 3B and 3D, type II). Three different siderophore mimics were used: hydroxypyranone, hydroxypyridone and catechol. Different amino acid linkers were explored for the possibility of hydrophobic interactions of their lipophilic side chains with hydrophobic amino-acid residues in the binding site, or cation- $\pi$ 
interactions with the Arg136 for compounds with phenylalanine and tyrosine linkers. The length of the linkers was also examined, with this extended by one carbon atom using $\beta$-alanine. The catechol siderophore mimic was attached via two different substitution patterns, to obtain either 3,4-dihydroxyphenyl-based or 2,3-dihydroxyphenyl-based conjugates. To compare the effects of the siderophore moiety on DNA gyrase inhibition, the compounds were also prepared with free amino $(11,12,29,30)$ or acetamido $(13,14,18-23)$ groups.
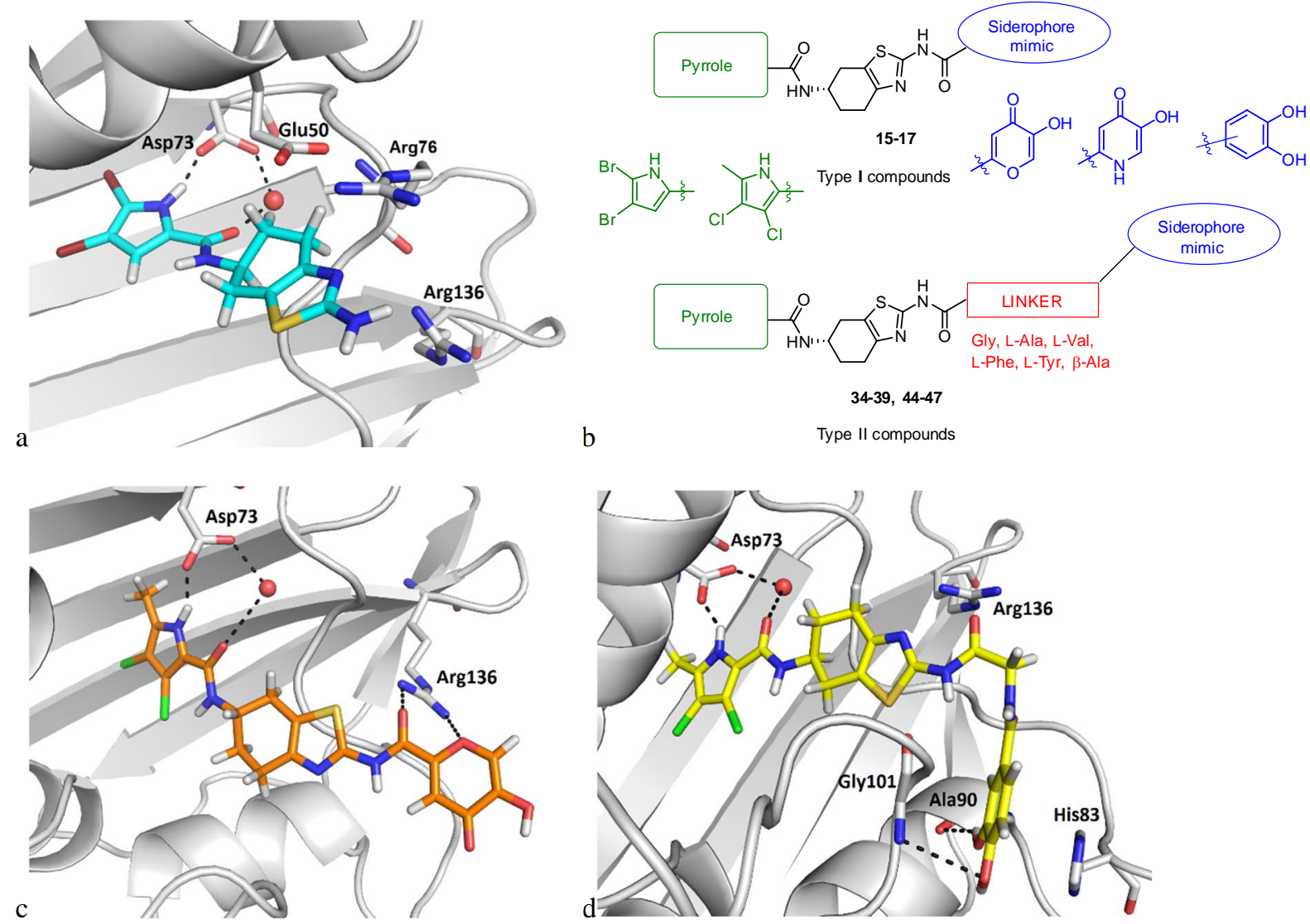

Figure 3. a) Molecular docking calculated binding mode of amine 11 (in cyan sticks) in the E. coli DNA gyrase ATP-binding site (PDB entry: 4DUH, in grey). b) Design of the DNA gyrase inhibitor-siderophore mimic conjugates, based on the binding mode of 11. c, d) Docking binding modes of compounds 17 (in orange sticks) and 34 (in yellow sticks) in the E. coli DNA gyrase ATP-binding site (in grey). For clarity, only selected amino acid residues are presented as sticks. Conserved water molecule is presented as a red sphere. Hydrogen bonds are presented as black dashed lines. 


\section{Chemistry}

The synthesis of the siderophore mimics was performed starting from kojic acid (Scheme 1, 4). First, the hydroxyl group directly attached to the ring was protected with either benzyl or paramethoxybenzyl functionality using benzyl bromide or 4-methoxybenzyl chloride under alkaline conditions, to obtain compounds 5 and $\mathbf{6}$. Subsequent oxidation of the aliphatic hydroxyl group with Jones reagent (chromium trioxide in sulfuric acid) yielded compounds $\mathbf{7}$ and $\mathbf{8}$. The removal of the $O$-benzyl group of 7 with catalytic hydrogenation using palladium on activated charcoal as catalyst resulted in siderophore mimic $\mathbf{9}$, and treating $\mathbf{8}$ with $25 \%$ aqueous ammonia gave compound $\mathbf{1 0 .}$<smiles>[R]c1ccc(COc2coc(CO)cc2=O)cc1</smiles>
kojic acid (4)

$$
5(\mathrm{R}=\mathrm{H})
$$
$6(\mathrm{R}=\mathrm{OMe})$

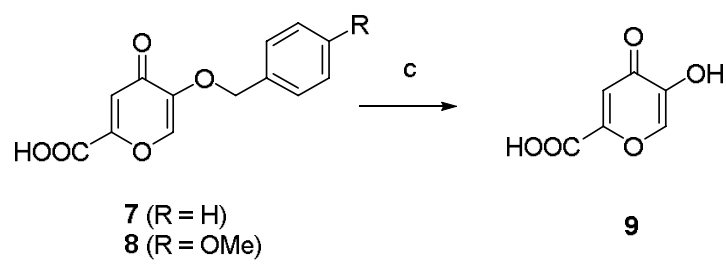

$d$<smiles>COc1ccc(COc2c[nH]c(C(=O)O)cc2=O)cc1</smiles>

Scheme 1. Reagents and conditions. (a) For 5: benzyl bromide, $10 \mathrm{M} \mathrm{NaOH}, \mathrm{MeOH}, 80^{\circ} \mathrm{C}$, overnight; for 6: 4methoxybenzyl chloride, $\mathrm{K}_{2} \mathrm{CO}_{3}$, DMF, $50{ }^{\circ} \mathrm{C}, 4 \mathrm{~h}$. $\left(\right.$ b) $\mathrm{CrO}_{3} / \mathrm{H}_{2} \mathrm{SO}_{4}$, acetone, 30 min at $-5{ }^{\circ} \mathrm{C}$, then $3 \mathrm{~h}$ at $20{ }^{\circ} \mathrm{C}$. (c) $\mathrm{H}_{2}, \mathrm{Pd} / \mathrm{C}$, abs. EtOH, $20{ }^{\circ} \mathrm{C}, 60$ min. (d) $25 \% \mathrm{NH}_{3}$ (aq), $80{ }^{\circ} \mathrm{C}, 4 \mathrm{~h}$.

All of these final compounds were synthesized from the parent $(S)-4,5,6,7-$ tetrahydrobenzo[d]thiazol-6-yl-1H-pyrrole-2-carboxamides 11 and 12 (Scheme 2), which were obtained according to previously reported procedures [10]. Also, acetamides $\mathbf{1 3}$ and $\mathbf{1 4}$ (Table 1) were obtained as reported previously [10]. Hydroxypyranone siderophore mimics in the form 
of carboxylic acids and either $O$-benzyl protected or not $(7,9)$ were coupled to the 2-amino group of 11 and 12 by 1-ethyl-3-(3-(dimethylamino)propyl)-carbodiimide (EDC)/ 1hydroxybenzotriazole (HOBt)-promoted amide bond formation, to yield compounds 15-17. In the same manner, compounds 18-23 were prepared using $N$-acetylated amino acids (Gly, L-Ala, L-Val, L-Phe, L-Tyr) and compounds 24-28, using $N$-Boc-protected amino acids (Gly, L-Ala, LVal, $\beta$-Ala). Removal of the Boc protecting group was performed with $4 \mathrm{M} \mathrm{HCl}$ in 1,4-dioxane or acetyl chloride in methanol, to obtain compounds $\mathbf{2 9 - 3 3}$, which were used in another EDC/ HOBt coupling to prepare analogs 34-43. Finally, removal of the para-methoxybenzyl protection group from compounds $\mathbf{4 0 - 4 3}$ with $1 \mathrm{M} \mathrm{HCl}$ in acetic acid resulted in compounds 44-47. 

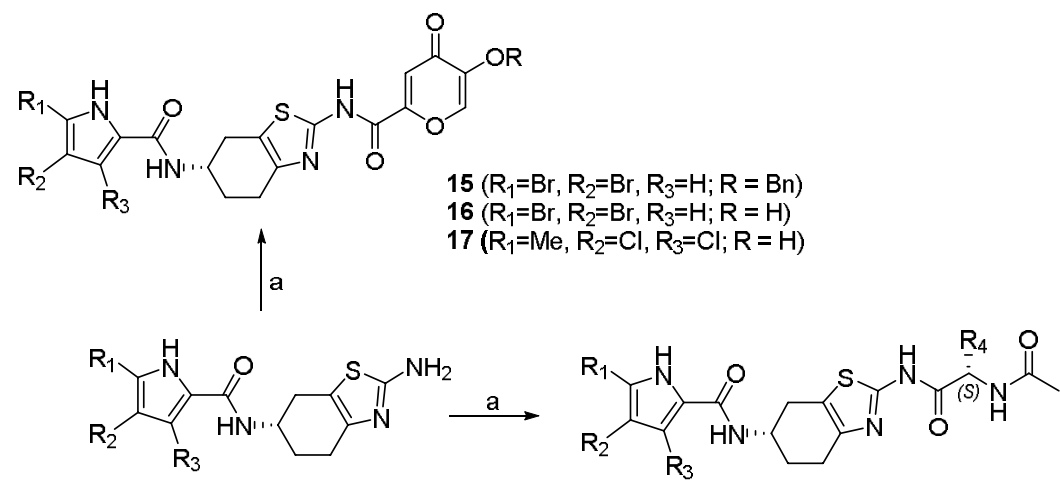

$11\left(\mathrm{R}_{1}=\mathrm{Br}, \mathrm{R}_{2}=\mathrm{Br}, \mathrm{R}_{3}=\mathrm{H}\right) \quad 18\left(\mathrm{R}_{1}=\mathrm{Br}, \mathrm{R}_{2}=\mathrm{Br}, \mathrm{R}_{3}=\mathrm{H} ; \mathrm{R}_{4}=\mathrm{H}\right)$ $12\left(R_{1}=\mathrm{Me}, \mathrm{R}_{2}=\mathrm{Cl}, \mathrm{R}_{3}=\mathrm{Cl}\right) \quad 19\left(\mathrm{R}_{1}=\mathrm{Me}, \mathrm{R}_{2}=\mathrm{Cl}, \mathrm{R}_{3}=\mathrm{Cl} ; \mathrm{R}_{4}=\mathrm{H}\right)$ $20\left(R_{1}=B r, R_{2}=B r, R_{3}=H ; R_{4}=M e\right)$ $\begin{array}{ll} & 21\left(R_{1}=B r, R_{2}=B r, R_{3}=H ; R_{4}=j-P r\right) \\ \text { a } & 22\left(R_{1}=B r, R_{2}=B r, R_{3}=H ; R_{4}=B n\right)\end{array}$<smiles>[R]c1[nH]c(C(=O)N[C@H]2CCc3nc(NC(=O)C([R])NC(=O)OC(C)(C)C)sc3C2)c([R])c1[R2]</smiles>
$23\left(\mathrm{R}_{1}=\mathrm{Br}, \mathrm{R}_{2}=\mathrm{Br}, \mathrm{R}_{3}=\mathrm{H} ; \mathrm{R}_{4}=p-\mathrm{OHBn}\right)$

\footnotetext{
$24\left(\mathrm{R}_{1}=\mathrm{Br}, \mathrm{R}_{2}=\mathrm{Br}, \mathrm{R}_{3}=\mathrm{H} ; \mathrm{R}_{4}=\mathrm{H} ; \mathrm{n}=1\right)$ $25\left(R_{1}=\mathrm{Me}, \mathrm{R}_{2}=\mathrm{Cl}, \mathrm{R}_{3}=\mathrm{Cl} ; \mathrm{R}_{4}=\mathrm{H} ; \mathrm{n}=1\right)$ $26\left(R_{1}=\mathrm{Me}, \mathrm{R}_{2}=\mathrm{Cl}, \mathrm{R}_{3}=\mathrm{Cl} ; \mathrm{R}_{4}=\mathrm{Me} ; \mathrm{n}=1\right)$ $27\left(\mathrm{R}_{1}=\mathrm{Me}, \mathrm{R}_{2}=\mathrm{Cl}, \mathrm{R}_{3}=\mathrm{Cl} ; \mathrm{R}_{4}=i-\mathrm{Pr} ; \mathrm{n}=1\right)$ $28\left(R_{1}=\mathrm{Me}, \mathrm{R}_{2}=\mathrm{Cl}, \mathrm{R}_{3}=\mathrm{Cl} ; \mathrm{R}_{4}=\mathrm{H} ; \mathrm{n}=2\right)$
}

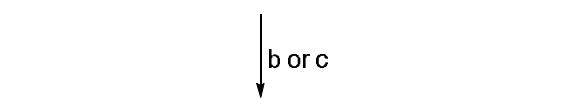

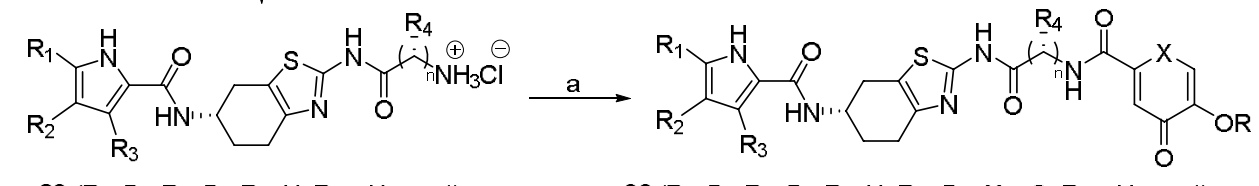

$29\left(\mathrm{R}_{1}=\mathrm{Br}, \mathrm{R}_{2}=\mathrm{Br}, \mathrm{R}_{3}=\mathrm{H} ; \mathrm{R}_{4}=\mathrm{H} ; \mathrm{n}=1\right)$ $30\left(\mathrm{R}_{1}=\mathrm{Me}, \mathrm{R}_{2}=\mathrm{Cl}, \mathrm{R}_{3}=\mathrm{Cl} ; \mathrm{R}_{4}=\mathrm{H} ; \mathrm{n}=1\right)$ $31\left(R_{1}=\mathrm{Me}, \mathrm{R}_{2}=\mathrm{Cl}, \mathrm{R}_{3}=\mathrm{Cl} ; \mathrm{R}_{4}=\mathrm{Me} ; \mathrm{n}=1\right)$ $32\left(\mathrm{R}_{1}=\mathrm{Me}, \mathrm{R}_{2}=\mathrm{Cl}, \mathrm{R}_{3}=\mathrm{Cl} ; \mathrm{R}_{4}=j-\mathrm{Pr} ; \mathrm{n}=1\right)$ $33\left(\mathrm{R}_{1}=\mathrm{Me}, \mathrm{R}_{2}=\mathrm{Cl}, \mathrm{R}_{3}=\mathrm{Cl} ; \mathrm{R}_{4}=\mathrm{H} ; \mathrm{n}=2\right)$

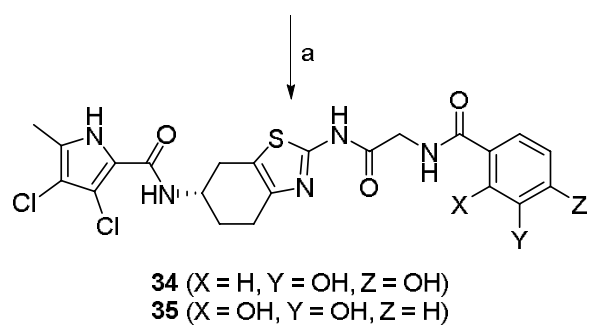

$36\left(\mathrm{R}_{1}=\mathrm{Br}, \mathrm{R}_{2}=\mathrm{Br}, \mathrm{R}_{3}=\mathrm{H} ; \mathrm{R}=\mathrm{Bn} ; \mathrm{X}=\mathrm{O} ; \mathrm{R}_{4}=\mathrm{H} ; \mathrm{n}=1\right)$ $37\left(R_{1}=\mathrm{Br}, \mathrm{R}_{2}=\mathrm{Br}, \mathrm{R}_{3}=\mathrm{H} ; \mathrm{R}=\mathrm{H} ; \mathrm{X}=\mathrm{O} ; \mathrm{R}_{4}=\mathrm{H} ; \mathrm{n}=1\right)$ $38\left(R_{1}=\mathrm{Me}, \mathrm{R}_{2}=\mathrm{Cl}, \mathrm{R}_{3}=\mathrm{Cl} ; \mathrm{R}=\mathrm{Bn} ; \mathrm{X}=\mathrm{O} ; \mathrm{R}_{4}=\mathrm{H} ; \mathrm{n}=1\right)$ $39\left(R_{1}=\mathrm{Me}, \mathrm{R}_{2}=\mathrm{Cl}, \mathrm{R}_{3}=\mathrm{Cl} ; \mathrm{R}=\mathrm{H} ; \mathrm{X}=\mathrm{O} ; \mathrm{R}_{4}=\mathrm{H} ; \mathrm{n}=1\right)$ $40\left(R_{1}=\mathrm{Me}, \mathrm{R}_{2}=\mathrm{Cl}, \mathrm{R}_{3}=\mathrm{Cl} ; \mathrm{R}=\mathrm{PMB} ; \mathrm{X}=\mathrm{N} ; \mathrm{R}_{4}=\mathrm{H} ; \mathrm{n}=1\right)$ $41\left(R_{1}=\mathrm{Me}, R_{2}=\mathrm{Cl}, \mathrm{R}_{3}=\mathrm{Cl} ; \mathrm{R}=\mathrm{PMB} ; \mathrm{X}=\mathrm{N} ; \mathrm{R}_{4}=\mathrm{Me} ; \mathrm{n}=1\right)$ $42\left(\mathrm{R}_{1}=\mathrm{Me}, \mathrm{R}_{2}=\mathrm{Cl}, \mathrm{R}_{3}=\mathrm{Cl} ; \mathrm{R}=\mathrm{PMB} ; \mathrm{X}=\mathrm{N} ; \mathrm{R}_{4}=j \mathrm{Pr} ; \mathrm{n}=1\right)$ $43\left(R_{1}=M e, R_{2}=C l, R_{3}=C l ; R=P M B ; X=N ; R_{4}=H ; n=2\right)$

Scheme 2. Reagents and conditions. (a) For 15-17: hydroxypyranone acid 7 or 9; for 18-23 corresponding $N$ acetylated amino acid derivative; for 24-28 corresponding $N$-Boc-protected amino acid derivative; for 34: 3,4dihydroxybenzoic acid; for 35: 2,3-dihydroxybenzoic acid; for 36-43: corresponding hydroxypyranone acid 7 or 9 or hydroxypyridone acid 10; EDC, HOBt, NMM, DMF, $0{ }^{\circ} \mathrm{C}$, then $20^{\circ} \mathrm{C}$, overnight, except $60 \mathrm{~h}$ for $\mathbf{1 5}$ and $\mathbf{2 5}$, $96 \mathrm{~h}$ for $\mathbf{1 7}$ and $72 \mathrm{~h}$ for $\mathbf{4 0}$; additional heating $5 \mathrm{~h}$ at $60^{\circ} \mathrm{C}$ for $\mathbf{1 6}$. (b) 24, acetyl chloride, $\mathrm{MeOH}, 90 \mathrm{~min}, 0^{\circ} \mathrm{C}$; then $20{ }^{\circ} \mathrm{C}$, 18 h. (c) Corresponding $N$-Boc-protected compounds 25-28, $4 \mathrm{M} \mathrm{HCl}$ in 1,4-dioxane, $20{ }^{\circ} \mathrm{C}, 16 \mathrm{~h} .(d)$ Corresponding 4-methoxybenzyl-protected compounds 40-43, $1 \mathrm{M} \mathrm{HCl}$ in acetic acid, $20{ }^{\circ} \mathrm{C}, 16 \mathrm{~h}$. 


\section{Results and Discussion}

\subsection{In vitro enzyme inhibition}

In total, 23 compounds $(\mathbf{1 5 - 2 3}, \mathbf{3 4 - 4 7})$ were synthesized and tested in the DNA gyrase supercoiling assay to determine their in vitro inhibitory activities against $E$. coli DNA gyrase. These data are presented in Figures 4 and 5, and Tables S1 and S2 as IC 50 values.

Overall, 12 of the compounds showed $E$. coli DNA gyrase inhibitory activities with $\mathrm{IC}_{50}$ $<1 \mu \mathrm{M}$, with seven of these at $<0.2 \mu \mathrm{M}$. Six of these seven were more potent inhibitors than the positive control novobiocin $\left(\mathrm{IC}_{50}, 0.17 \mu \mathrm{M}\right): \mathbf{1 9}, \mathbf{2 0}, \mathbf{2 1}, \mathbf{3 4}, 35$ and 39. For the synthesized GyrB inhibitors bearing a siderophore mimic, six of 17 showed $\mathrm{IC}_{50}$ against $E$. coli DNA gyrase $<0.5 \mu \mathrm{M}$, with the most potent compound 34 with an $\mathrm{IC}_{50}$ of $0.058 \mu \mathrm{M}$ (Figure 5 , Table S2). Moreover, this $\mathrm{IC}_{50}$ for $\mathbf{3 4}$ is comparable to that of the oxalyl acid $\mathbf{1}$ (Figure 2), which is one of the most potent 4,5,6,7-tetrahydrobenzo[d]thiazole-based DNA gyrase inhibitors described to date [10].

The E. coli DNA gyrase inhibition was improved by acylation of the 2-amino group of the central scaffold (Figure 4, Table S1), as the acetamides $\mathbf{1 3}$ and $\mathbf{1 4}\left(\mathrm{IC}_{50}, 0.15 \mu \mathrm{M}, 0.48 \mu \mathrm{M}\right.$, respectively) were more potent than their free amino counterparts $\mathbf{1 1}$ and $\mathbf{1 2}\left(\mathrm{IC}_{50},>10 \mu \mathrm{M}\right)$. The same was seen for the acetamides $\mathbf{1 8}$ and $\mathbf{1 9}$ and the amines 29 and $\mathbf{3 0}$ with glycine as the linker (Figure 4, Table S1). Amino acids with small side chains as linkers (Gly, L-Ala, L-Val, $\beta$-Ala) were well tolerated ( $\mathrm{IC}_{50}, 0.10-0.52 \mu \mathrm{M}$ for $\mathbf{1 8 - 2 1}$ ), while bulkier side chains in the linkers (L-Phe, L-Tyr) led to a loss of activity ( $\mathrm{IC}_{50}, 7.0 \mu \mathrm{M}, 2.8 \mu \mathrm{M}$ for $\mathbf{2 2}, \mathbf{2 3}$, respectively). Therefore, only DNA gyrase inhibitors with non-aromatic amino acids as linkers were considered for the synthesis of the conjugates.

In the type I compounds (Figure 5, Table S2), the dibromopyrrole analog $\mathbf{1 6}\left(\mathrm{IC}_{50}, 0.18\right.$ $\mu \mathrm{M})$ was significantly more potent than its dichloromethylpyrrole counterpart $\mathbf{1 7}\left(\mathrm{IC}_{50}, 2.1\right.$ 
$\mu \mathrm{M})$. The attached hydroxypyranone siderophore mimic did not affect the binding, as starting compound $13\left(\mathrm{IC}_{50}, 0.15 \mu \mathrm{M}\right)$ and its conjugate analog 16 had comparable $\mathrm{IC}_{50}$ values. Furthermore, the additional benzyl group in $\mathbf{1 5}\left(\mathrm{IC}_{50}, 3.4 \mu \mathrm{M}\right)$ largely reduced the affinity for E. coli DNA gyrase, which was also observed in the type II compounds $\mathbf{3 6}$ and $\mathbf{3 8}$ (Figure 5, Table S2, IC $50,2.0 \mu \mathrm{M}, 2.1 \mu \mathrm{M}$, respectively). Catechol-based conjugates $\mathbf{3 4}$ (IC $50,0.058 \mu \mathrm{M})$ and $35\left(\mathrm{IC}_{50}, 0.11 \mu \mathrm{M}\right)$ with a glycine linker were more potent $E$. coli DNA gyrase inhibitors than the hydroxypyranone $37\left(\mathrm{IC}_{50}, 0.69 \mu \mathrm{M}\right)$ and the hydroxypyridinone $44\left(\mathrm{IC}_{50}, 1.6 \mu \mathrm{M}\right)$ and had comparable activities to the hydroxypyranone conjugate $39\left(\mathrm{IC}_{50}, 0.090 \mu \mathrm{M}\right)$. Increasing the linker length of 44 in the $\beta$-Ala analog $47\left(\mathrm{IC}_{50}, 0.35 \mu \mathrm{M}\right)$ improved the DNA gyrase inhibition. Moreover, introduction of a methyl $\left(45 ; \mathrm{IC}_{50}, 0.26 \mu \mathrm{M}\right)$ or $i$-Pr $\left(46 ; \mathrm{IC}_{50}, 0.64 \mu \mathrm{M}\right)$ group into the linker also increased the DNA gyrase inhibitory activities.

Figure 4. Enzyme inhibition data of the $(S)-4,5,6,7$-tetrahydrobenzo[d]thiazol-6-yl-1H-pyrrole-2-carboxamidebased compounds against Escherichia coli DNA gyrase.

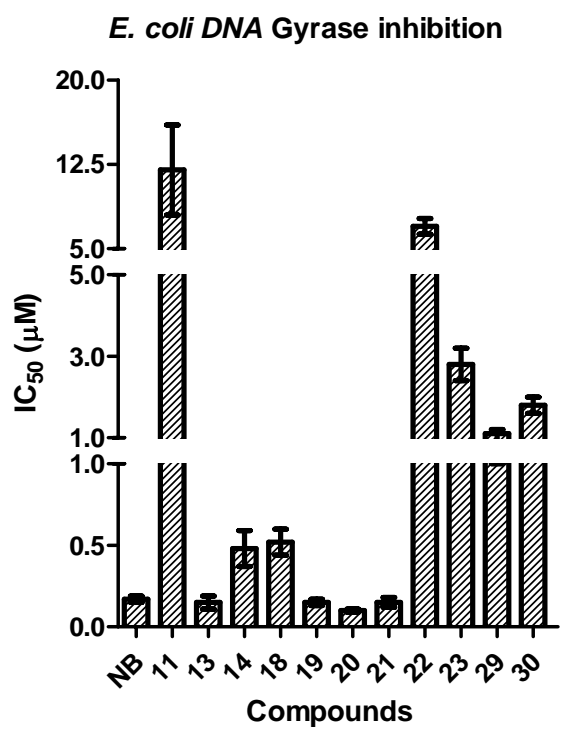


Figure 5. Enzyme inhibition data of DNA gyrase inhibitor - siderophore mimic conjugates against Escherichia coli DNA gyrase.

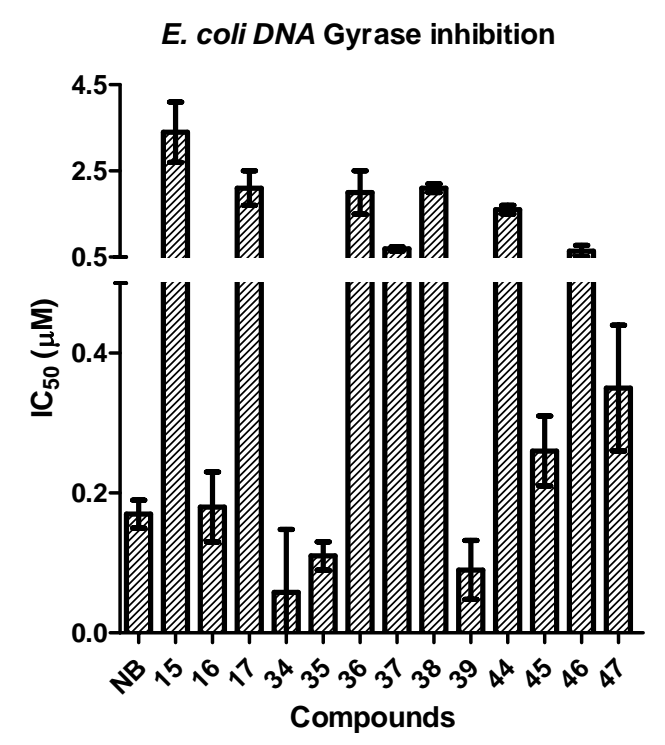

\subsection{Antibacterial activities}

All of these compounds were evaluated for their in vitro antibacterial activities against two Gram-positive (Enterococcus faecalis ATCC 29212, S. aureus ATCC 25923 or 29213) and two Gram-negative (E. coli ATCC 25922, P. aeruginosa ATCC 27853) bacterial strains. In addition, they were tested against two E. coli mutant strains: JD17464, as an lpxC deletion mutant with an impaired outer membrane; and JW5503, as a tolC deletion mutant with a defective efflux pump. Preliminary tests were performed at $50 \mu \mathrm{M}$, and growth inhibition was measured after $24 \mathrm{~h}$ incubation. These data are presented in Figures S1 and S2 in the Supplementary Information. MICs were determined in dose-response assays for compounds that showed $>90 \%$ growth in the preliminary assay (Table S3) [27]. The compounds containing the siderophore mimics were evaluated also against E. coli (ATCC 25922), P. aeruginosa (ATCC 27853) and A. baumannii (ATCC 19606) in cation-adjusted Mueller-Hinton broth (CAMHB) as iron-depleted (ID-CAMHB) or when supplemented with iron, to determine the effects of the attached siderophore mimics on the antibacterial activities in iron depleted conditions. These data are presented in Table S4 in the Supplementary Information as 
percentages of bacterial growth inhibition at $50 \mu \mathrm{M}$ of the tested compounds and in Figure 6 . Data of growth inhibition for non-conjugated compounds 13, 14, 18, 20, 21 and 29 against $E$. coli ATCC 25922, in CAMHB and ID-CAMHB, are also shown in Table S4. Since there is no difference in activity, any possible direct effect due to different culture medium composition can be excluded.

These antibacterial activity assays revealed activity of compounds $\mathbf{3 0}$ and $\mathbf{3 5}$ against the Gram-positive bacterial strain of E. faecalis with MICs of $11 \mu \mathrm{g} / \mathrm{mL}$ and $14 \mu \mathrm{g} / \mathrm{mL}$, respectively. On the other hand, tested compounds were generally inactive against the wildtype Gram-negative bacterial strains in iron-supplemented medium. However, weak correlations between in vitro inhibitory activities and antibacterial properties have also been reported previously for other structural classes of DNA gyrase B inhibitors [8]. In addition, the inhibitory activities here in the iron-depleted medium were generally also weak against all of the bacterial strains tested, with only two compounds $(\mathbf{4 5}, \mathbf{4 6})$ showing $>50 \%$ growth inhibition against A. baumannii (Figure 6). For compounds 35, 44, 45 and 47 we observed an opposite effect on bacterial growth of $P$. aeruginosa, since they showed a drop in growth inhibition in iron-supplemented compared to iron-depleted medium. Several groups have investigated the effect of introducing siderophores mimics into the structure of fluoroquinolones, well-known DNA gyrase inhibitors, but the activities of most of these conjugates were also not greater compared to those of the parent antibiotics, or in many cases activities were even lost [28].

However, increases in the inhibitory effects were seen for iron-depleted versus ironsupplemented media for almost all of the compounds tested against wild-type E. coli, presumably as a consequence of the attached siderophore mimics. Moreover, inhibition against the $\Delta$ tolC E. coli JW5503 strain was seen (Supplementary Figures S1, S2 and Table S3). For this $E$. coli mutant with a defective efflux pump, the most potent bacterial growth inhibition was for compound 19 with an MIC of $1 \mu \mathrm{g} / \mathrm{mL}$. Among the siderophore mimic conjugates, the 
most promising activities were shown by the catechol derivatives 34 and 35, with MICs of 14 $\mu \mathrm{g} / \mathrm{mL}$ against the $\Delta$ tolC $E$. coli JW5503 strain, which is more effective than for the best compounds from the first generation 4,5,6,7-tetrahydrobenzo[d]thiazoles [10]. In contrast, none of the tested compounds showed significantly improved antibacterial activity against $\triangle \operatorname{lpxC} E$. coli JD17464 compared to wild-type strain. These data suggest that efflux problems, but not cell wall penetration, remain the issue in terms of the weak antibacterial activities of the 4,5,6,7tetrahydrobenzo $[d]$ thiazole derivatives.
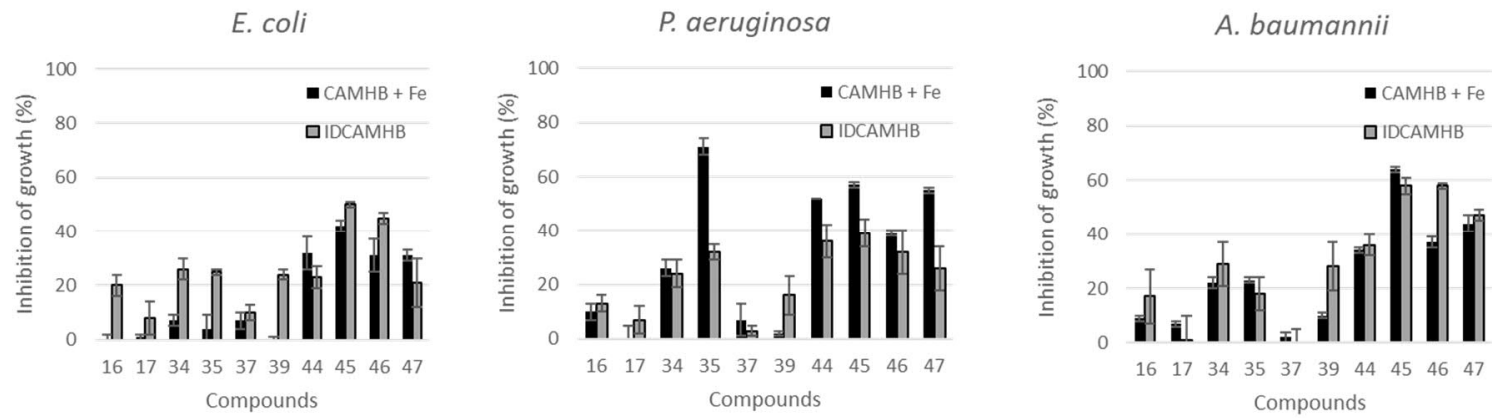

Figure 6. Antibacterial activity of DNA gyrase B inhibitor-siderophore mimic conjugates against $E$. coli, $P$. aeruginosa and A. baumannii in iron-depleted (ID-CAMHB) and iron-supplemented CAMHB media at $50 \mu \mathrm{M}$ concentration. Cefiderocol MICs in CAMHB were $0.25,1.0$, and $2.0 \mu \mathrm{g} / \mathrm{mL}$, and in ID-CAMHB $0.25,0.5$ and $0.25 \mu \mathrm{g} / \mathrm{ml}$ against $E$. coli, $P$. aeruginosa and A. baumannii, respectively (complying with approved CLSI values) [29]. Ceftazidime was used as a negative control and MICs were $0.25,2$, and $>4 \mu \mathrm{g} / \mathrm{mL}$ both in CAMHB and IDCAMHB against E. coli, $P$. aeruginosa and A. baumannii, respectively.

Recent studies revealed that not only siderophore-mimic moieties, but also other groups present in the molecule could contribute to iron chelation [30, 31]. For example, BAL30072 (Figure 1) has been found to form compact complex in solution comprising two BAL30072 molecules and one $\mathrm{Fe}^{3+}$ ion. In this complex sulfate group of BAL30072 participates as a chelating functionality in addition to the dihydroxypyridone moiety [30]. In another study with (pre)acinetobactin, a siderophore secreted by A. baumannii, the same ligand to metal stoichiometry in the solution was found to be favorable in low iron conditions and such complex 
is readily recognized and transported by the outer membrane (OM) transporter into the periplasm of A. baumannii. Interestingly, only one oxygen from a catechol moiety of preacinetobactin participates in complex formation, together with three other atoms in the scaffold [31]. Thus, more focused investigation of the interactions of our DNA gyrase inhibitorsiderophore mimic conjugates with $\mathrm{Fe}^{3+}$ is required in the future to completely elucidate stoichiometry of these complexes as well as to identify functional groups responsible for iron chelation. Moreover, since the interactions with the OM transporters also play a key role in circumventing poor permeation in Gram-negative bacteria, further studies on affinity and binding mode of our conjugates in OM transporters are needed, such as recently published study on enterobactin-iron complex with $P$. aeruginosa OM transporter PfeA [32]. Inappropriate shape and size of our conjugates and consequentially their poor affinity toward OM transporters might be a reason for only slight improvements in antibacterial activities in iron-depleted versus iron-supplemented media against tested wild-type Gram-negative bacterial strains. Moreover, the solving of the efflux problems should be considered as equally important to achieve improved antibacterial activities against Gram-negative bacterial strains.

\section{Conclusion}

These new 4,5,6,7-tetrahydrobenzo $[d]$ thiazole derivatives conjugated with three types of siderophore mimics were designed, synthesized and evaluated in biological assays. In vitro enzyme inhibition of E. coli DNA gyrase revealed that compounds $\mathbf{3 4}$ and $\mathbf{3 5}$, which include a catechol siderophore mimic moiety, are more potent inhibitors than the positive control novobiocin, with $\mathrm{IC}_{50}$ in the low nanomolar range $(<0.2 \mu \mathrm{M})$. The most potent among all of the compounds tested was $\mathbf{3 4}$, with $\mathrm{IC}_{50}$ of $0.058 \mu \mathrm{M}$, which is comparable to the best $4,5,6,7-$ tetrahydrobenzo[ $d]$ thiazole-based DNA gyrase inhibitors described to date [10]. This shows 
that it is possible to design potent DNA gyrase inhibitor-siderophore mimic conjugates with non-cleavable linkers. In vitro antibacterial assays showed no significant activities against wildtype Gram-negative bacterial strains. For the efflux pump mutant strain, both of these potent catechol mimic conjugates 34 and 35 showed a MIC of $14 \mu \mathrm{g} / \mathrm{mL}$ against E. coli JW5503, demonstrating that active efflux together with permeability remains the issue for these compounds. Both $\mathbf{3 4}$ and $\mathbf{3 5}$ also showed minor improvements in their antibacterial activities against wild-type E. coli in iron-depleted medium. Therefore, the use of catechols as siderophore mimics appears to be a promising approach for further investigations into DNA gyrase inhibitor-siderophore mimic conjugates with sufficient antibacterial activity against wild-type Gram-negative bacterial strains.

\section{Experimental Section}

\subsection{Materials and Methods}

The chemicals were obtained from Acros Organics (Geel, Belgium), Sigma-Aldrich (St. Louis, MO, USA), TCI Europe N.V. (Zwijndrecht, Belgium) and Apollo Scientific (Stockport, UK), and were used without further purification. Analytical TLC was performed on silica gel Merck $60 \mathrm{~F}_{254}$ plates $(0.25 \mathrm{~mm})$, with visualization with UV light and spray reagents. Column chromatography was carried out on silica gel 60 (particle size, 240-400 mesh). HPLC analyses were performed on: $(i)$ an Agilent Technologies 1100 instrument with a UV-VIS detector (G1365B), a thermostat (G1316A), an autosampler (G1313A) and a C18 column (Eclipse Plus; $5 \mu \mathrm{m}, 4.6 \times 150 \mathrm{~mm}$; Agilent); (ii) a Thermo Scientific Dionex Ultimate 3000 Binary Rapid Separation LC System (Thermo Fisher Scientific, Waltham, MA, USA) with an autosampler, a binary pump system, a photodiode array detector, a thermostated column compartment and a C18 column (Zorbax Extend; $3.5 \mu \mathrm{m}, 4.6 \times 150 \mathrm{~mm}$; Agilent). The following gradient elution 
was used with mobile phases A (0.1\% trifluoroacetic acid in water) and B (acetonitrile): 0-16 $\min , 95 \%-5 \% \mathrm{~A} ; 16-21 \mathrm{~min}, 5 \% \mathrm{~A}$. The flow rate was $1.0 \mathrm{~mL} / \mathrm{min}$, and the injection volume was $20 \mu \mathrm{L}$. All of the tested compounds were $\geq 95 \%$ pure by HPLC. Melting points were determined on a Reichert hot-stage microscope, and are uncorrected. ${ }^{1} \mathrm{H}$ and ${ }^{13} \mathrm{C}$ NMR spectra were recorded at $400 \mathrm{MHz}$ and $100 \mathrm{MHz}$, respectively, on a spectrometer (AVANCE III 400; Bruker Corporation, Billerica, MA, USA) in DMSO- $d_{6}$ or $\mathrm{CDCl}_{3}$ solutions, with TMS as the internal standard. Mass spectra were obtained using Advion expression CMS mass spectrometer (Advion Inc., Ithaca, USA) and high resolution mass spectra were obtained using a Q-TOF Premier mass spectrometer (Micromass, Waters, Manchester, UK) or an Exactive Plus Orbitrap mass spectrometer (Thermo Fischer Scientific Inc., Waltham, MA, USA). Optical rotations were measured on a polarimeter (241 MC; Perkin-Elmer). The reported values for specific rotation were the means of five successive measurements, using an integration time of $5 \mathrm{~s}$.

\subsection{Synthesis}

\subsubsection{Synthesis of siderophore mimics}

Siderophore mimics were synthesized according to the reported procedures $[33,34]$.

6.2.1.1 General procedure A. Chromium trioxide $(2.8 \mathrm{mmol})$ was suspended in water $(1 \mathrm{~mL})$ and concentrated sulfuric acid $(0.3 \mathrm{~mL})$ was added dropwise on an ice bath. The mixture was quantitatively introduced into a solution of corresponding alcohol (1 mmol), which was previously dissolved in acetone $(10 \mathrm{~mL})$ with additional heating and then cooled on an ice bath. Reaction mixture was stirred for $30 \mathrm{~min}$ at $0{ }^{\circ} \mathrm{C}$ and then for $3 \mathrm{~h}$ at room temperature. The precipitate was filtered off through a layer of Celite and mother liquid was evaporated under reduced pressure. Water $(2 \mathrm{~mL})$ was added to the residue and precipitate formed was filtered off and dried at $40^{\circ} \mathrm{C}$. 
6.2.1.1.1 5-(Benzyloxy)-2-(hydroxymethyl)-4H-pyran-4-one (5). Kojic acid (10.0 g, 70.4 mmol) was suspended in methanol $(80 \mathrm{~mL})$ and $10 \mathrm{M} \mathrm{NaOH}(7.74 \mathrm{~mL}, 77.4 \mathrm{mmol})$ was added. Then benzyl bromide $(9.61 \mathrm{~mL}, 80.9 \mathrm{mmol})$ was added dropwise and reaction mixture was stirred at $80{ }^{\circ} \mathrm{C}$ overnight. Reaction mixture was allowed to cool to room temperature and solvent was evaporated under reduced pressure. Acetone $(12 \mathrm{~mL})$ and water $(120 \mathrm{~mL})$ were added to the crude residue and precipitate was formed, which was filtered off and dried at $40{ }^{\circ} \mathrm{C}$. Yield 16.845 g (99.0 \%); yellow solid; m. p. $123-125{ }^{\circ} \mathrm{C} ;{ }^{1} \mathrm{H}$ NMR $\left(400 \mathrm{MHz}, \mathrm{CDCl}_{3}\right) \delta 4.48(\mathrm{~s}, 2 \mathrm{H}$, $\left.\mathrm{CH}_{2}\right), 5.08\left(\mathrm{~s}, 2 \mathrm{H}, \mathrm{CH}_{2}\right), 6.57(\mathrm{~s}, 1 \mathrm{H}$, pyran-H), 7.31-7.42 (m, 5H, Ar-H), $7.56(\mathrm{~s}, 1 \mathrm{H}$, pyran-H) ppm, signal for $\mathrm{OH}$ not seen in the spectrum.

6.2.1.1.2 2-(Hydroxymethyl)-5-((4-methoxybenzyl)oxy)-4H-pyran-4-one (6). Kojic acid (5.0 g, $35.2 \mathrm{mmol})$ and potassium carbonate $(10.7 \mathrm{~g}, 77.4 \mathrm{mmol})$ were suspended in dry $N, N$ dimethylformamide (DMF) $(80 \mathrm{~mL})$. The round-bottom flask was filled with argon and 4methoxybenzyl chloride $(5.23 \mathrm{~mL}, 38.7 \mathrm{mmol})$ was added dropwise. Reaction mixture was stirred at $50{ }^{\circ} \mathrm{C}$ for $4 \mathrm{~h}$. Then DMF was evaporated under reduced pressure and water $(100 \mathrm{~mL})$ was added. A precipitate was formed, which was filtered off and dried at $60{ }^{\circ} \mathrm{C}$. Yield $8.145 \mathrm{~g}$ (88 \%); yellow solid; m. p. $114-116{ }^{\circ} \mathrm{C} ;{ }^{1} \mathrm{H}$ NMR (400 MHz, DMSO-d $) \delta 3.77$ (s, $3 \mathrm{H}, \mathrm{CH}_{3}$ ), $4.30\left(\mathrm{~d}, 2 \mathrm{H}, J=4.0 \mathrm{~Hz}, \underline{\mathrm{C}}_{2} \mathrm{OH}\right), 4.86\left(\mathrm{~s}, 2 \mathrm{H}, \mathrm{CH}_{2}\right), 5.75(\mathrm{br} \mathrm{s}, 1 \mathrm{H}, \mathrm{OH}), 6.32(\mathrm{t}, 1 \mathrm{H}, J=0.9$ Hz, pyran-H), 6.92-6.99 (m, 2H, Ar-H), 7.31-7.39 (m, 2H, Ar-H), 8.16 (s, 1H, pyran-H) ppm; HRMS ESI ${ }^{+} \mathrm{m} / \mathrm{z}$ for $\mathrm{C}_{14} \mathrm{H}_{15} \mathrm{O}_{5}\left([\mathrm{M}+\mathrm{H}]^{+}\right)$: calcd 263.0919, found 263.0921 .

6.2.1.1.3 5-(Benzyloxy)-4-oxo-4H-pyran-2-carboxylic acid (7). Prepared from 5 (5.0 g, 21.5 mmol $)$ and chromium trioxide $(6.02 \mathrm{~g}, 60.2 \mathrm{mmol})$ according to the general procedure $\mathbf{A}$. Yield 3.437 g (65 \%); yellow solid; m. p. $162-164{ }^{\circ} \mathrm{C} ;{ }^{1} \mathrm{H}$ NMR (400 MHz, DMSO-d 6 ) $\delta 4.99$ (s, $\left.2 \mathrm{H}, \mathrm{CH}_{2}\right), 6.93(\mathrm{~s}, 1 \mathrm{H}$, pyran-H), 7.34-7.47 (m, 5H, Ar-H), 8.37 (s, 1H, pyran-H) ppm, signal for $\mathrm{COOH}$ not seen in the spectrum; HRMS ESI ${ }^{-} \mathrm{m} / \mathrm{z}$ for $\mathrm{C}_{13} \mathrm{H}_{9} \mathrm{O}_{5}\left([\mathrm{M}-\mathrm{H}]^{-}\right)$: calcd 245.0450, found 245.0451. 
6.2.1.1.4 5-((4-Methoxybenzyl)oxy)-4-oxo-4H-pyran-2-carboxylic acid (8). Prepared from 6 (2.05 g, $7.82 \mathrm{mmol})$ and chromium trioxide (2.19 g, $21.9 \mathrm{mmol})$ according to the general procedure A. Precipitate was additionally washed with hot $\mathrm{MeOH}(2 \times 30 \mathrm{~mL})$. Yield $0.980 \mathrm{~g}$ (45 \%); yellow solid; m. p. $165-167{ }^{\circ} \mathrm{C} ;{ }^{1} \mathrm{H}$ NMR (400 MHz, DMSO-d 6 ) $\delta 3.77$ (s, 3H, $\mathrm{CH}_{3}$ ), $4.90\left(\mathrm{~s}, 2 \mathrm{H}, \mathrm{CH}_{2}\right), 6.93$ (s, 1H, pyran-H); 6.94-7.00 (m, 2H, Ar-H), 7.34-7.40 (m, 2H, Ar-H), $8.36\left(\mathrm{~s}, 1 \mathrm{H}\right.$, pyran-H) ppm; HRMS ESI ${ }^{-} \mathrm{m} / \mathrm{z}$ for $\mathrm{C}_{14} \mathrm{H}_{11} \mathrm{O}_{6}\left([\mathrm{M}-\mathrm{H}]^{-}\right)$: calcd 275. 0556, found 275.0562 .

6.2.1.1.5 5-Hydroxy-4-oxo-4H-pyran-2-carboxylic acid (9). Compound 7 (0.400 g, 1.22 mmol) was dissolved in dry ethanol $(80 \mathrm{~mL})$ and put under argon. Then $5 \% \mathrm{~m} / \mathrm{m}$ of $\mathrm{Pd} / \mathrm{C}(0.020 \mathrm{~g})$ was added and reaction mixture was stirred for $1 \mathrm{~h}$ at room temperature under hydrogen atmosphere. Then $\mathrm{Pd} / \mathrm{C}$ was filtered off and the solvent evaporated under reduced pressure. Yield 0.236 g (93 \%); yellow amorphous powder; ${ }^{1} \mathrm{H}$ NMR (400 MHz, DMSO- $\left.d_{6}\right) \delta 6.96$ (s, $1 \mathrm{H}$, pyran-H); $8.21(\mathrm{~s}, 1 \mathrm{H}$, pyran-H), 9.67 (br s, $1 \mathrm{H}, \mathrm{OH}) \mathrm{ppm} ; \mathrm{HRMS} \mathrm{ESI}^{-} \mathrm{m} / \mathrm{z}$ for $\mathrm{C}_{6} \mathrm{H}_{3} \mathrm{O}_{5}$ ([M-H]'): calcd 154.9986, found 154.9981.

\subsection{5-((4-Methoxybenzyl)oxy)-4-oxo-1,4-dihydropyridine-2-carboxylic acid (10).} Compound 8 (0.390 g, $1.41 \mathrm{mmol})$ was dissolved in $25 \%$ aqueous ammonia (10 $\mathrm{mL})$ and refluxed for $4 \mathrm{~h}$ at $80{ }^{\circ} \mathrm{C}$. Then reaction mixture was concentrated to about $5 \mathrm{~mL}$ and slowly acidified with concentrated hydrochloric acid to $\mathrm{pH}=2$. Precipitate formed was filtered off and dried under reduced pressure (co-evaporation with $2 \times 5 \mathrm{~mL}$ diethyl ether). Yield $0.325 \mathrm{~g}$ (84 $\%$ ); white amorphous solid; ${ }^{1} \mathrm{H}$ NMR (400 MHz, DMSO- $\left.d_{6}\right) \delta 3.76\left(\mathrm{~s}, 3 \mathrm{H}, \mathrm{CH}_{3}\right), 5.12(\mathrm{~s}, 2 \mathrm{H}$, $\mathrm{CH}_{2}$ ), 6.96 (m, 2H, Ar-H), 7.24 (br s, 1H, pyridine-H), 7.39 (m, 2H, Ar-H), 7.93 (br s, 1H, pyridine-H) ppm, signals for $\mathrm{NH}$ and $\mathrm{COOH}$ not seen in the spectrum; HRMS ESI ${ }^{-} \mathrm{m} / \mathrm{z}$ for $\mathrm{C}_{14} \mathrm{H}_{12} \mathrm{NO}_{5}\left([\mathrm{M}-\mathrm{H}]^{-}\right)$: calcd 274.0715, found 274.0723.

\subsubsection{Synthesis of compounds}


6.2.2.1 General procedure B. A solution of carboxylic acid (1 mmol) in $N, N$ dimethylformamide $(10 \mathrm{~mL})$ was cooled to $0{ }^{\circ} \mathrm{C}$ and then EDC $(1.2 \mathrm{mmol})$ and $\mathrm{HOBt}(1.3$ mmol) were added. $\mathrm{pH}$ was adjusted to 8 with $\mathrm{N}$-methylmorpholine and the reaction mixture stirred for $20 \mathrm{~min}$ at $0{ }^{\circ} \mathrm{C}$. Then amine $(1 \mathrm{mmol})$ was added and reaction mixture stirred overnight at room temperature. The solvent was evaporated in vacuo and the residue dissolved in ethyl acetate $(30 \mathrm{~mL})$ and washed successively with $1 \%$ citric acid $(2 \times 30 \mathrm{~mL})$, saturated aqueous $\mathrm{NaHCO}_{3}$ solution $(2 \times 30 \mathrm{~mL})$, and brine $(30 \mathrm{~mL})$. The organic phase was dried over $\mathrm{Na}_{2} \mathrm{SO}_{4}$, filtered and solvent evaporated under reduced pressure.

6.2.2.1.1

(S)-N-(2-(5-(Benzyloxy)-4-oxo-4H-pyran-2-carboxamido)-4,5,6,7tetrahydrobenzo[d]thiazol-6-yl)-4,5-dibromo-1H-pyrrole-2-carboxamide (15). Prepared from $11(0.300 \mathrm{~g}, 0.714 \mathrm{mmol})$ and $7(0.176 \mathrm{~g}, 0.714 \mathrm{mmol})$ according to the general procedure $\mathbf{B}$. Product was purified by flash column chromatography using dichloromethane/methanol (15:1) as eluent. Yield $0.094 \mathrm{~g}(20 \%)$; yellow solid; m. p. $168-170{ }^{\circ} \mathrm{C} ;[\alpha]_{\mathrm{D}}+32.8(c 0.19, \mathrm{DMF}) ;{ }^{1} \mathrm{H}$ NMR (400 MHz, DMSO-d6) $\delta$ 1.83-1.94 (m, 1H, H $\left.\mathrm{A}_{\mathrm{A}}-7\right), 1.95-2.08\left(\mathrm{~m}, 1 \mathrm{H}, \mathrm{H}_{\mathrm{B}}-7\right), 2.57-2.66$ (m, $\left.1 \mathrm{H}, \mathrm{H}_{\mathrm{A}}-5\right), 2.70-2.78\left(\mathrm{~m}, 2 \mathrm{H}, \mathrm{H}_{\mathrm{B}}-5, \mathrm{H}_{\mathrm{A}}-4\right), 2.94-3.06\left(\mathrm{~m}, 1 \mathrm{H}, \mathrm{H}_{\mathrm{B}}-4\right), 4.16-4.31(\mathrm{~m}, 1 \mathrm{H}$, C $\underline{H N H}), 5.02\left(\mathrm{~s}, 2 \mathrm{H}, \mathrm{OCH}_{2}\right), 7.01(\mathrm{~d}, 1 \mathrm{H}, J=2.6 \mathrm{~Hz}$, pyrrole-H), 7.10 (s, 1H, pyran-H), 7.357.49 (m, 5H, Ar-H), $8.12(\mathrm{~d}, 1 \mathrm{H}, J=7.8 \mathrm{~Hz}, \mathrm{CHNH}), 8.33$ (s, 1H, pyran-H), 12.72 (d, 1H, $J=$ 2,5 Hz, pyrrole-NH) ppm; ${ }^{13} \mathrm{C}$ NMR (100 MHz, DMSO- $\left.d_{6}\right) \delta 27.8,70.1,97.2,104.0,112.4$, 114.7, 127.4, 127.7, 127.9, 135.3, 140.3, 147.5, $157.8 \mathrm{ppm} ;$ HRMS ESI $\mathrm{m} / \mathrm{z}$ for $\mathrm{C}_{25} \mathrm{H}_{19} \mathrm{Br}_{2} \mathrm{~N}_{4} \mathrm{O}_{5} \mathrm{~S}\left([\mathrm{M}-\mathrm{H}]^{-}\right)$: calcd 644.9443, found 644.9439; HPLC: $\mathrm{t}_{\mathrm{r}} 13.15 \min (95 \%$ at 254 $\mathrm{nm})$.

6.2.2.1.2 (S)-4,5-Dibromo-N-(2-(5-hydroxy-4-oxo-4H-pyran-2-carboxamido)-4,5,6,7tetrahydrobenzo[d]thiazol-6-yl)-1H-pyrrole-2-carboxamide (16). Prepared from 11 (0.300 g, $0.714 \mathrm{mmol})$ and $\mathbf{9}(0.111 \mathrm{~g}, 0.714 \mathrm{mmol})$ according to the general procedure $\mathbf{B}$. After addition of $10 \%$ citric acid and ethyl acetate, precipitate was formed, which was filtered off and dried. 
Crude residue was suspended in a small volume of ethyl acetate, heated until boiling and filtered off. Product was dried in vacuo. Yield $0.044 \mathrm{~g}(11 \%)$; brown amorphous solid; $[\alpha]_{\mathrm{D}}+41.5(c$ $0.20, \mathrm{DMF}) ;{ }^{1} \mathrm{H}$ NMR $\left(400 \mathrm{MHz}, \mathrm{DMSO}-d_{6}\right) \delta 1.81-1.96\left(\mathrm{~m}, 1 \mathrm{H}, \mathrm{H}_{\mathrm{A}}-7\right), 1.96-2.07\left(\mathrm{~m}, 1 \mathrm{H}, \mathrm{H}_{\mathrm{B}^{-}}\right.$

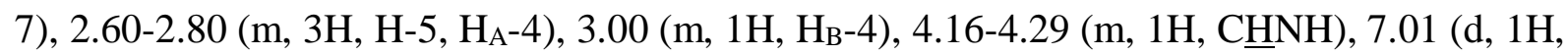
$J=2.4 \mathrm{~Hz}$, pyrrole-H), $7.12(\mathrm{~s}, 1 \mathrm{H}$, pyran-H), $8.12(\mathrm{~d}, 1 \mathrm{H}, J=7.6 \mathrm{~Hz}, \mathrm{CHN} \underline{\mathrm{H}}), 8.18(\mathrm{~s}, 1 \mathrm{H}$, pyran-H), $9.62(\mathrm{~s}, 1 \mathrm{H}, \mathrm{OH}), 12.72\left(\mathrm{~d}, 1 \mathrm{H}, J=2.4 \mathrm{~Hz}\right.$, pyrrole-NH) ppm; ${ }^{13} \mathrm{C}$ NMR $(100 \mathrm{MHz}$, DMSO-d $d_{6} \delta 27.9,28.4,44.9,97.8,104.6,113.0,114.1,128.0,139.7,147.5,158.4,173.7$ ppm; HRMS ESI ${ }^{-} \mathrm{m} / \mathrm{z}$ for $\mathrm{C}_{18} \mathrm{H}_{13} \mathrm{Br}_{2} \mathrm{~N}_{4} \mathrm{O}_{5} \mathrm{~S}\left([\mathrm{M}-\mathrm{H}]^{-}\right)$: calcd 554.8968, found 554.8984; HPLC: $\mathrm{t}_{\mathrm{r}}$ $10.77 \min (95 \%$ at $254 \mathrm{~nm})$.

6.2.2.1.3

(S)-3,4-Dichloro-N-(2-(5-hydroxy-4-oxo-4H-pyran-2-carboxamido)-4,5,6,7tetrahydrobenzo[d]thiazol-6-yl)-5-methyl-1H-pyrrole-2-carboxamide (17). Prepared from 12 $(0.126 \mathrm{~g}, 0.365 \mathrm{mmol})$ and $\mathbf{9}(0.057 \mathrm{~g}, 0.365 \mathrm{mmol})$ according to the general procedure $\mathbf{B}$. After addition of $10 \%$ citric acid, precipitate was formed, which was filtered off and washed with ethyl acetate $(3 \times 10 \mathrm{~mL})$ and hot methanol $(3 \times 1 \mathrm{~mL})$. Yield $0.030 \mathrm{~g}(17 \%)$; brown amorphous solid; $[\alpha]_{\mathrm{D}}-17.7\left(c\right.$ 0.19, DMSO); ${ }^{1} \mathrm{H}$ NMR (400 MHz, DMSO- $\left.d_{6}\right) \delta 1.90-2.11(\mathrm{~m}, 2 \mathrm{H}, \mathrm{H}-7)$, $2.19\left(\mathrm{~s}, 3 \mathrm{H}, \mathrm{CH}_{3}\right), 2.65-2.84\left(\mathrm{~m}, 3 \mathrm{H}, \mathrm{H}-5, \mathrm{H}_{\mathrm{A}}-4\right), 3.02\left(\mathrm{~d}, 1 \mathrm{H}, J_{1}=14.1 \mathrm{~Hz}, \mathrm{H}_{\mathrm{B}}-4\right), 4.20-4.35$

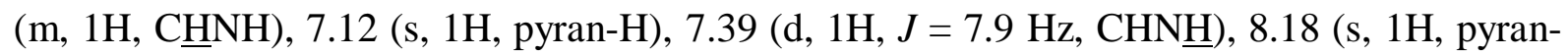
H), $9.63(\mathrm{~s}, 1 \mathrm{H}, \mathrm{OH}), 12.02\left(\mathrm{~s}, 1 \mathrm{H}\right.$, pyrrole-NH) ppm; ${ }^{13} \mathrm{C}$ NMR (100 MHz, DMSO- $\left.d_{6}\right) \delta 10.7$, 27.5, 28.3, 44.9, 108.0, 110.0, 114.1, 119.2, 127.3, 139.7, 147.5, 158.3, 173.7 ppm; HRMS ESI ${ }^{+}$ $\mathrm{m} / \mathrm{z}$ for $\mathrm{C}_{19} \mathrm{H}_{17} \mathrm{~N}_{4} \mathrm{O}_{5} \mathrm{SCl}_{2}\left([\mathrm{M}+\mathrm{H}]^{+}\right)$: calcd 483.0291, found 483.0282; HPLC: $\mathrm{t}_{\mathrm{r}} 11.42 \mathrm{~min}$ (95 $\%$ at $254 \mathrm{~nm})$.

6.2.2.1.4 (S)-N-(2-(2-Acetamidoacetamido)-4,5,6,7-tetrahydrobenzo[d]thiazol-6-yl)-4,5dibromo-1H-pyrrole-2-carboxamide (18). Prepared from $11(0.200 \mathrm{~g}, 0.476 \mathrm{mmol})$ and $\mathrm{N}$ acetylglycine $(0.058 \mathrm{~g}, 0.476 \mathrm{mmol})$ according to the general procedure B. Product was purified by flash column chromatography using ethyl acetate to ethyl acetate/methanol (5:2) as eluent. 
Yield 0.086 g (35 \%); yellow solid; m. p. $196-198^{\circ} \mathrm{C} ;[\alpha]_{\mathrm{D}}-7.4(c 0.30, \mathrm{MeOH}) ;{ }^{1} \mathrm{H}$ NMR (400 MHz, DMSO-d $d_{6} \delta 1.83-1.91\left(\mathrm{~m}, 4 \mathrm{H}, \mathrm{H}_{\mathrm{A}}-7, \mathrm{CH}_{3}\right), 1.96-2.03\left(\mathrm{~m}, 1 \mathrm{H}, \mathrm{H}_{\mathrm{B}}-7\right), 2.58-2.77(\mathrm{~m}, 3 \mathrm{H}$, $\left.\mathrm{H}-5, \mathrm{H}_{\mathrm{A}}-4\right), 2.99\left(\mathrm{dd}, 1 \mathrm{H}, J_{l}=15.5 \mathrm{~Hz}, J_{2}=5.2 \mathrm{~Hz}, \mathrm{H}_{\mathrm{B}}-4\right), 3.94\left(\mathrm{~d}, 2 \mathrm{H}, J=5.8 \mathrm{~Hz}, \mathrm{C}_{2} \mathrm{NH}\right)$,

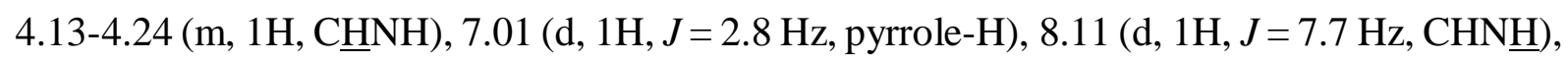
$8.26\left(\mathrm{t}, 1 \mathrm{H}, J=5.9 \mathrm{~Hz}, \mathrm{CH}_{2} \mathrm{NH}\right), 11.98(\mathrm{~s}, 1 \mathrm{H}, \mathrm{NHCO}), 12.71(\mathrm{~d}, 1 \mathrm{H}, J=2.6 \mathrm{~Hz}$, pyrrole- $\mathrm{NH})$ ppm; ${ }^{13} \mathrm{C}$ NMR (100 MHz, DMSO- $\left.d_{6}\right) \delta 22.3,24.6,28.4,28.6,41.9,45.3,97.8,104.5,112.9$, 119.3, 128.0, 143.5, 155.3, 158.4, 167.9, 169.8 ppm; HRMS ESI- m/z for $\mathrm{C}_{16} \mathrm{H}_{16} \mathrm{Br}_{2} \mathrm{~N}_{5} \mathrm{O}_{3} \mathrm{~S}$ ([M$\mathrm{H}]^{-}$): calcd 515.9341, found 515.9346; HPLC: $\mathrm{t}_{\mathrm{r}} 11.48 \min (99 \%$ at $254 \mathrm{~nm})$.

6.2.2.1.5 (S)-N-(2-(2-Acetamidoacetamido)-4,5,6,7-tetrahydrobenzo[d]thiazol-6-yl)-3,4dichloro-5-methyl-1H-pyrrole-2-carboxamide (19). Prepared from 12 (0.150 g, 0.435 mmol) and $N$-acetylglycine $(0.051 \mathrm{~g}, 0.435 \mathrm{mmol})$ according to the general procedure $\mathbf{B}$. Crude product was crystalized from methanol. Yield $0.078 \mathrm{~g}(40 \%)$; yellow solid; m. p. $168-171{ }^{\circ} \mathrm{C} ;[\alpha]_{\mathrm{D}}$ -9.6 (c 0.13, DMF); ${ }^{1} \mathrm{H}$ NMR (400 MHz, DMSO- $\left.d_{6}\right) \delta 1.88$ (s, 3H, $\left.\mathrm{CH}_{3}\right), 1.91-2.07(\mathrm{~m}, 2 \mathrm{H}, \mathrm{H}-$ 7), 2.19 (s, 3H, pyrrole- $\left.\mathrm{CH}_{3}\right), 2.65-2.78\left(\mathrm{~m}, 3 \mathrm{H}, \mathrm{H}-5, \mathrm{H}_{\mathrm{A}}-4\right), 3.01\left(\mathrm{~m}, 1 \mathrm{H}, \mathrm{H}_{\mathrm{B}}-4\right), 3.94(\mathrm{~d}, 2 \mathrm{H}$, $\left.J=5.8 \mathrm{~Hz}, \underline{\mathrm{C}}_{2} \mathrm{NH}\right), 4.20-4.31(\mathrm{~m}, 1 \mathrm{H}, \mathrm{C} \underline{\mathrm{HNH}}), 7.32(\mathrm{~d}, 1 \mathrm{H}, J=7.8 \mathrm{~Hz}, \mathrm{CHN} \underline{\mathrm{H}}), 8.27(\mathrm{t}, 1 \mathrm{H}$,

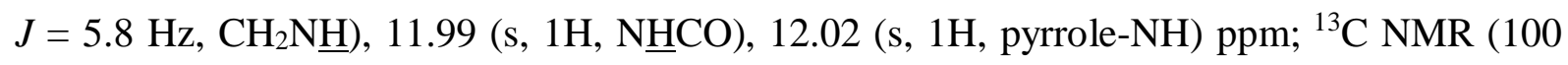
MHz, DMSO-d $d_{6} \delta 11.1,22.8,24.7,28.5,28.8,42.4,45.6,108.5,110.5,112.6,119.7,127.8$, 143.8, 155.8, 158.7, 168.4, 170.3 ppm; HRMS ESI ${ }^{-} \mathrm{m} / \mathrm{z}$ for $\mathrm{C}_{17} \mathrm{H}_{18} \mathrm{~N}_{5} \mathrm{O}_{3} \mathrm{SCl}_{2}\left([\mathrm{M}-\mathrm{H}]^{-}\right)$: calcd 442.0507, found 442.0496; HPLC: $\mathrm{t}_{\mathrm{r}} 10.55 \mathrm{~min}$ (97\% at $254 \mathrm{~nm}$ ).

6.2.2.1.6 N-((S)-2-((S)-2-Acetamidopropanamido)-4,5,6,7-tetrahydrobenzo[d]thiazol-6-yl)4,5-dibromo-1H-pyrrole-2-carboxamide (20). Prepared from $11(0.160 \mathrm{~g}, 0.381 \mathrm{mmol})$ and $\mathrm{N}$ acetyl-L-alanine $(0.050 \mathrm{~g}, 0.381 \mathrm{mmol})$ according to the general procedure B. Product was purified by flash column chromatography using ethyl acetate to ethyl acetate/methanol $(5: 2)$ as eluent. Yield $0.081 \mathrm{~g}(40 \%)$; yellow solid; m. p. $160-162{ }^{\circ} \mathrm{C} ;[\alpha]_{\mathrm{D}}+10.3(c 0.18, \mathrm{DMF}) ;{ }^{1} \mathrm{H}$ NMR (400 MHz, DMSO- $\left.d_{6}\right) \delta 1.27\left(\mathrm{dd}, 3 \mathrm{H}, J_{1}=7.2 \mathrm{~Hz}, J_{2}=1.6 \mathrm{~Hz}, \mathrm{CHCH}_{3}\right), 1.81-1.90$ (m, 
$\left.4 \mathrm{H}, \mathrm{H}_{\mathrm{A}}-7, \mathrm{COCH}_{3}\right), 1.94-2.03\left(\mathrm{~m}, 1 \mathrm{H}, \mathrm{H}_{\mathrm{B}}-7\right), 2.59-2.75\left(\mathrm{~m}, 3 \mathrm{H}, \mathrm{H}-5, \mathrm{H}_{\mathrm{A}}-4\right), 2.98\left(\mathrm{~m}, 1 \mathrm{H}, \mathrm{H}_{\mathrm{B}}-\right.$

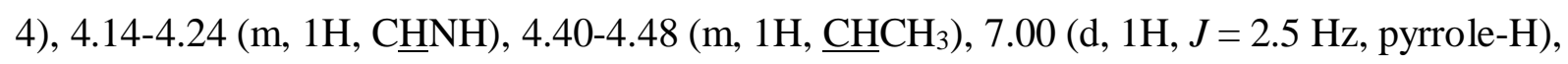
8.09 (t, 1H, $J=7.2 \mathrm{~Hz}, \mathrm{CHN} \underline{\mathrm{H}}), 8.25(\mathrm{~d}, 1 \mathrm{H}, J=6.8 \mathrm{~Hz}, \mathrm{CHN} \underline{\mathrm{H}}), 11.98(\mathrm{~d}, 1 \mathrm{H}, J=1.9 \mathrm{~Hz}$, pyrrole-NH), 12.70 (s, 1H, NHCO) ppm; ${ }^{13} \mathrm{C}$ NMR (100 MHz, DMSO- $\left.d_{6}\right) \delta 18.1,22.8,25.1$, 28.8, 29.1, 45.7, 48.8, 98.3, 105.0, 113.4, 119.9, 128.6, 144.0, 155.9, 158.9, 169.8, 171.9 ppm; HRMS ESI ${ }^{-} \mathrm{m} / \mathrm{z}$ for $\mathrm{C}_{17} \mathrm{H}_{18} \mathrm{Br}_{2} \mathrm{~N}_{5} \mathrm{O}_{3} \mathrm{~S}\left([\mathrm{M}-\mathrm{H}]^{-}\right)$: calcd 529.9497, found 529.9496; HPLC: $\mathrm{t}_{\mathrm{r}}$ $11.77 \mathrm{~min}(97.6 \%$ at $254 \mathrm{~nm})$.

6.2.2.1.7 N-((S)-2-((S)-2-Acetamido-3-methylbutanamido)-4,5,6,7-tetrahydrobenzo[d]thiazol6-yl)-4,5-dibromo-1H-pyrrole-2-carboxamide (21). Prepared from 11 (0.180 g, 0.428 mmol) and $N$-acetyl-L-valine $(0.068 \mathrm{~g}, 0.428 \mathrm{mmol})$ according to the general procedure B. Product was purified by flash column chromatography using dichloromethane/methanol (20:1) as eluent. Yield $0.047 \mathrm{~g}(20 \%)$; white solid; m. p. $180-182{ }^{\circ} \mathrm{C} ;{ }^{1} \mathrm{H}$ NMR (400 MHz, DMSO- $\left.d_{6}\right) \delta$ $0.88\left(\mathrm{t}, 6 \mathrm{H}, J=7.5 \mathrm{~Hz}, 2 \times \mathrm{CH}_{3}\right), 1.80-1.92\left(\mathrm{~m}, 4 \mathrm{H}, \mathrm{H}_{\mathrm{A}}-7, \mathrm{COCH}_{3}\right), 1.94-2.06\left(\mathrm{~m}, 2 \mathrm{H}, \mathrm{H}_{\mathrm{B}}-7\right.$, $\left.\mathrm{C} \underline{\mathrm{H}}\left(\mathrm{CH}_{3}\right)_{2}\right), 2.59-2.74\left(\mathrm{~m}, 3 \mathrm{H}, \mathrm{H}-5, \mathrm{H}_{\mathrm{A}}-4\right), 2.98\left(\mathrm{dd}, 1 \mathrm{H}, J_{l}=14.4 \mathrm{~Hz}, J_{2}=5.4 \mathrm{~Hz}, \mathrm{H}_{\mathrm{B}}-4\right), 4.16-$

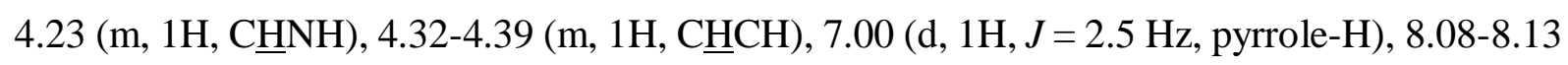
$(\mathrm{m}, 2 \mathrm{H}, 2 \times \mathrm{CHN} \underline{\mathrm{H}}), 12.04(\mathrm{~s}, 1 \mathrm{H}, \mathrm{NHCO}), 12.70(\mathrm{~d}, 1 \mathrm{H}, J=2.6 \mathrm{~Hz}$, pyrrole-NH) ppm; HRMS $\mathrm{ESI}^{-} \mathrm{m} / \mathrm{z}$ for $\mathrm{C}_{19} \mathrm{H}_{22} \mathrm{Br}_{2} \mathrm{~N}_{5} \mathrm{O}_{3} \mathrm{~S}$ ([M-H] $\left.]^{-}\right)$: calcd 557.9810, found 557.9823; HPLC: $\mathrm{t}_{\mathrm{r}} 12.38 \mathrm{~min}$ $(100 \%$ at $254 \mathrm{~nm})$.

6.2.2.1.8

$N-((S)-2-((S)-2-A c e t a m i d o-3-p h e n y l p r o p a n a m i d o)-4,5,6,7-$ tetrahydrobenzo[d]thiazol-6-yl)-4,5-dibromo-1H-pyrrole-2-carboxamide (22). Prepared from $11(0.180 \mathrm{~g}, 0.428 \mathrm{mmol})$ and $N$-acetyl-L-phenylalanine $(0.089 \mathrm{~g}, 0.428 \mathrm{mmol})$ according to the general procedure B. Product was purified by flash column chromatography using dichloromethane/methanol (20:1) as eluent. Yield $0.023 \mathrm{~g}(9 \%)$; white solid; m. p. 143-145 ${ }^{\circ} \mathrm{C}$; $[\alpha]_{\mathrm{D}}+10.3(c \mathrm{0.18}, \mathrm{DMF}) ;{ }^{1} \mathrm{H} \mathrm{NMR}\left(400 \mathrm{MHz}, \mathrm{DMSO}-d_{6}\right) \delta 1.77-1.91\left(\mathrm{~m}, 4 \mathrm{H}, \mathrm{H}_{\mathrm{A}}-7, \mathrm{COCH}_{3}\right)$, 1.95-2.04 (m, 1H, H $\left.\mathrm{H}_{\mathrm{B}}-7\right), 2.60-2.74\left(\mathrm{~m}, 3 \mathrm{H}, \mathrm{H}-5, \mathrm{H}_{\mathrm{A}}-4\right), 2.84\left(\mathrm{dd}, 1 \mathrm{H}, J_{1}=13.1 \mathrm{~Hz}, J_{2}=3.2\right.$ 
$\left.\mathrm{Hz}, \mathrm{H}_{\mathrm{B}}-4\right)$, 2.95-3.06 (m, 2H, PhC $\left.\underline{\mathrm{H}}_{2}\right), 4.14-4.24$ (m, 1H, $\left.\mathrm{C} \underline{\mathrm{HNH}}\right), 4.68-4.74$ (m, 1H, $\left.\underline{\mathrm{HCH}}_{2}\right)$, $7.01(\mathrm{~d}, 1 \mathrm{H}, J=2.6 \mathrm{~Hz}$, pyrrole-H), 7.18-7.31 (m, 5H, Ar-H), 8.07-8.13 (m, 1H, CHN $\underline{\mathrm{H}}), 8.33$ (d, $1 \mathrm{H}, J=7.8 \mathrm{~Hz}, \mathrm{CHN} \underline{\mathrm{H}}), 12.18$ (s, 1H, NHCO), 12.71 (d, 1H, $J=1.9 \mathrm{~Hz}$, pyrrole-NH) ppm; HRMS ESI ${ }^{+} \mathrm{m} / \mathrm{z}$ for $\mathrm{C}_{23} \mathrm{H}_{24} \mathrm{Br}_{2} \mathrm{~N}_{5} \mathrm{O}_{3} \mathrm{~S}\left([\mathrm{M}+\mathrm{H}]^{+}\right)$: calcd 607.9961, found 607.9959; HPLC: $\mathrm{t}_{\mathrm{r}}$ $13.08 \min (100 \%$ at $254 \mathrm{~nm})$.

6.2.2.1.9

$N-((S)-2-((S)-2-A c e t a m i d o-3-(4-h y d r o x y p h e n y l) p r o p a n a m i d o)-4,5,6,7-$ tetrahydrobenzo[d]thiazol-6-yl)-4,5-dibromo-1H-pyrrole-2-carboxamide (23). Prepared from $11(0.100 \mathrm{~g}, 0.238 \mathrm{mmol})$ and $\mathrm{N}$-acetyl-L-tyrosine $(0.053 \mathrm{~g}, 0.238 \mathrm{mmol})$ according to the general procedure B. Product was purified by flash column chromatography using dichloromethane/methanol (20:1) as eluent. Yield $0.037 \mathrm{~g}(25 \%)$; white solid; m. p. 188-190 ${ }^{\circ} \mathrm{C} ;[\alpha]_{\mathrm{D}}+13.2(c 0.08, \mathrm{DMF}) ;{ }^{1} \mathrm{H}$ NMR $\left(400 \mathrm{MHz}, \mathrm{DMSO}-d_{6}\right) \delta 1.80\left(\mathrm{~s}, 3 \mathrm{H}, \mathrm{COCH}_{3}\right), 1.83-$ $1.91\left(\mathrm{~m}, 1 \mathrm{H}, \mathrm{H}_{\mathrm{A}}-7\right), 1.95-2.04\left(\mathrm{~m}, 1 \mathrm{H}, \mathrm{H}_{\mathrm{B}}-7\right), 2.59-2.74\left(\mathrm{~m}, 4 \mathrm{H}, \mathrm{H}-5, \mathrm{H}_{\mathrm{A}}-4, \mathrm{PhCH}_{2}-\mathrm{H}_{\mathrm{A}}\right), 2.87-$ $2.92\left(\mathrm{~m}, 1 \mathrm{H}, \mathrm{PhC}_{\mathrm{H}_{2}}-\mathrm{H}_{\mathrm{B}}\right), 2.99\left(\mathrm{dd}, 1 \mathrm{H}, J_{1}=15.8 \mathrm{~Hz}, J_{2}=5.1 \mathrm{~Hz}, \mathrm{H}_{\mathrm{B}}-4\right), 4.13-4.24(\mathrm{~m}, 1 \mathrm{H}$, C $\underline{H N H}), 4.57-4.67\left(\mathrm{~m}, 1 \mathrm{H}, \underline{\mathrm{HCH}}_{2}\right), 6.64(\mathrm{~d}, 2 \mathrm{H}, J=8.5 \mathrm{~Hz}, \mathrm{Ar}-\mathrm{H}), 7.01\left(\mathrm{dd}, 1 \mathrm{H}, J_{1}=2.6 \mathrm{~Hz}\right.$, $J_{2}=1.1 \mathrm{~Hz}$, pyrrole-H), $7.07(\mathrm{~d}, 2 \mathrm{H}, J=8.5 \mathrm{~Hz}, \mathrm{Ar}-\mathrm{H}), 8.10\left(\mathrm{dd}, 1 \mathrm{H}, J_{l}=7.4 \mathrm{~Hz}, J_{2}=2.1 \mathrm{~Hz}\right.$, CHN프), $8.25(\mathrm{~d}, 1 \mathrm{H}, J=7.8 \mathrm{~Hz}, \mathrm{CHN} \underline{\mathrm{H}}), 9.21(\mathrm{~d}, 1 \mathrm{H}, J=1.1 \mathrm{~Hz}, \mathrm{OH}), 12.11(\mathrm{~s}, 1 \mathrm{H}, \mathrm{NHCO})$, $12.70\left(\mathrm{~d}, 1 \mathrm{H}, J=2.7 \mathrm{~Hz}\right.$, pyrrole-NH) ppm; ${ }^{13} \mathrm{C}$ NMR (100 MHz, DMSO- $\left.d_{6}\right) \delta 22.8,25.1,28.9$, $37.1,45.7,55.0,98.3,105.0,113.4,115.4,119.9,127.8,128.5,130.6,144.0,155.7,156.4$, 158.9, 169.9, 170.9 ppm; HRMS ESI ${ }^{-} \mathrm{m} / \mathrm{z}$ for $\mathrm{C}_{23} \mathrm{H}_{22} \mathrm{Br}_{2} \mathrm{~N}_{5} \mathrm{O}_{4} \mathrm{~S}\left([\mathrm{M}-\mathrm{H}]^{-}\right)$: calcd 621.9759, found 621.9741; HPLC: $\operatorname{tr}_{\mathrm{r}} 12.19 \min (97.6 \%$ at $254 \mathrm{~nm})$.

6.2.2.1.10 tert-Butyl (S)-(2-((6-(4,5-dibromo-1H-pyrrole-2-carboxamido)-4,5,6,7tetrahydrobenzo[d]thiazol-2-yl)amino)-2-oxoethyl)carbamate (24). Prepared from 11 (0.918 g, $2.19 \mathrm{mmol})$ and $N$-Boc-glycine $(0.383 \mathrm{~g}, 2.19 \mathrm{mmol})$ according to the general procedure $\mathbf{B}$. Product was purified by flash column chromatography using dichloromethane/methanol (20:1) as eluent. Yield $0.672 \mathrm{~g}(53 \%)$; yellow solid; m. p. $156-158{ }^{\circ} \mathrm{C} ;[\alpha]_{\mathrm{D}}-2.96(c 0.15, \mathrm{MeOH})$; 
${ }^{1} \mathrm{H}$ NMR (400 MHz, DMSO-d $) \delta 1.35$ (s, 9H, $\left.3 \times \mathrm{CH}_{3}\right), 1.79-1.93\left(\mathrm{~m}, 1 \mathrm{H}, \mathrm{H}_{\mathrm{A}}-7\right), 1.95-2.04$ $\left(\mathrm{m}, 1 \mathrm{H}, \mathrm{H}_{\mathrm{B}}-7\right), 2.57-2.76\left(\mathrm{~m}, 3 \mathrm{H}, \mathrm{H}-5, \mathrm{H}_{\mathrm{A}}-4\right), 2.98\left(\mathrm{dd}, 1 \mathrm{H}, J_{1}=15.6 \mathrm{~Hz}, J_{2}=4.9 \mathrm{~Hz}, \mathrm{H}_{\mathrm{B}}-4\right)$, $3.81\left(\mathrm{~d}, 2 \mathrm{H}, J=6.2 \mathrm{~Hz}, \underline{\mathrm{C}}_{2} \mathrm{NH}\right), 4.12-4.26(\mathrm{~m}, 1 \mathrm{H}, \underline{\mathrm{CHNH}}), 7.00(\mathrm{~d}, 1 \mathrm{H}, J=1.9 \mathrm{~Hz}$, pyrrole-

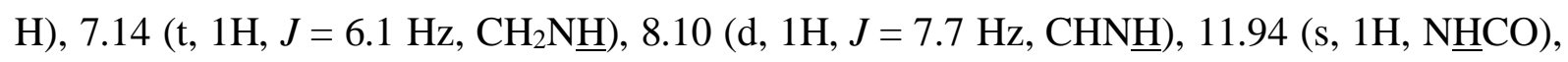
12.70 (s, $1 \mathrm{H}$, pyrrole-NH) ppm; ${ }^{13} \mathrm{C}$ NMR (100 MHz, DMSO- $\left.d_{6}\right) \delta 24.6,28.1,28.4,28.6,42.9$, 45.2, 78.2, 97.8, 104.5, 112.9, 119.2, 128.0, 143.5, 155.3, 155.8, 158.4, 168.2 ppm; HRMS ESI ${ }^{-}$ $\mathrm{m} / \mathrm{z}$ for $\mathrm{C}_{19} \mathrm{H}_{22} \mathrm{Br}_{2} \mathrm{~N}_{5} \mathrm{O}_{4} \mathrm{~S}\left([\mathrm{M}-\mathrm{H}]^{-}\right)$: calcd 573.9759, found 573.9752; HPLC: $\mathrm{t}_{\mathrm{r}} 13.23$ min (96 \% at $254 \mathrm{~nm})$.

6.2.2.1.11 tert-Butyl (S)-(2-((6-(3,4-dichloro-5-methyl-1H-pyrrole-2-carboxamido)-4,5,6,7tetrahydrobenzo[d]thiazol-2-yl)amino)-2-oxoethyl)carbamate (25). Prepared from 12 (0.220 g, $0.637 \mathrm{mmol})$ and $\mathrm{N}$-Boc-glycine $(0.112 \mathrm{~g}, 0.637 \mathrm{mmol})$ according to the general procedure $\mathbf{B}$. Yield $0.128 \mathrm{~g}$ (40 \%); yellow amorphous solid; $[\alpha]_{\mathrm{D}}+3.3(c 0.43, \mathrm{DMF}) ;{ }^{1} \mathrm{H}$ NMR $(400 \mathrm{MHz}$, DMSO- $\left.d_{6}\right) \delta 1.30$ and $1.39\left(2 \times \mathrm{s}, 9 \mathrm{H}, 3 \times \mathrm{CH}_{3}\right), 1.89-2.07(\mathrm{~m}, 2 \mathrm{H}, \mathrm{H}-7), 2.18(\mathrm{~s}, 3 \mathrm{H}$, pyrrole$\left.\mathrm{CH}_{3}\right), 2.66-2.77\left(\mathrm{~m}, 3 \mathrm{H}, \mathrm{H}-5, \mathrm{H}_{\mathrm{A}}-4\right), 3.02\left(\mathrm{dd}, 1 \mathrm{H}, J_{1}=15.9 \mathrm{~Hz}, J_{2}=5.0 \mathrm{~Hz}, \mathrm{H}_{\mathrm{B}}-4\right), 3.81(\mathrm{~d}$, $\left.2 \mathrm{H}, J=6.1 \mathrm{~Hz}, \underline{\mathrm{C}}_{2} \mathrm{NH}\right), 4.21-4.32(\mathrm{~m}, 1 \mathrm{H}, \mathrm{C} \underline{\mathrm{HNH}}), 7.14\left(\mathrm{t}, 1 \mathrm{H}, J=6.1 \mathrm{~Hz}, \mathrm{CH}_{2} \mathrm{~N} \underline{\mathrm{H}}\right), 7.32(\mathrm{~d}$,

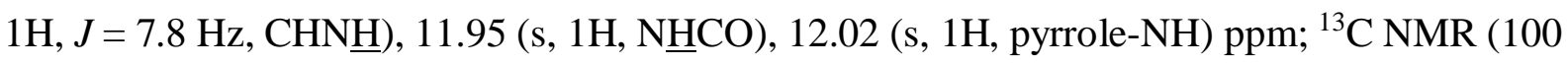
MHz, DMSO-d6) $\delta$ 10.6, 24.1, 27.9, 28.1, 28.2, 42.9, 45.1, 78.1, 107.9, 109.8, 119.0, 119.1, 127.3, 143.3, 155.3, 155.8, 158.1, $168.1 \mathrm{ppm}$; $\mathrm{HRMS} \mathrm{ESI}^{+} \mathrm{m} / \mathrm{z}$ for $\mathrm{C}_{20} \mathrm{H}_{26} \mathrm{~N}_{5} \mathrm{O}_{4} \mathrm{SCl}_{2}\left([\mathrm{M}+\mathrm{H}]^{+}\right)$: calcd 502.1077, found 502.1074; HPLC: $\mathrm{t}_{\mathrm{r}} 11.09 \mathrm{~min}(97.7 \%$ at $254 \mathrm{~nm})$.

6.2.2.1.12 tert-Butyl ((S)-1-(((S)-6-(3,4-dichloro-5-methyl-1H-pyrrole-2-carboxamido)4,5,6,7-tetrahydrobenzo[d]thiazol-2-yl)amino)-1-oxopropan-2-yl)carbamate (26). Prepared from $12(0.301 \mathrm{~g}, 0.872 \mathrm{mmol})$ and $N$-Boc-L-alanine $(0.165 \mathrm{~g}, 0.872 \mathrm{mmol})$ according to the general procedure B. Product was purified by flash column chromatography using dichloromethane/methanol (20:1) as eluent. Yield 0.216 g (48 \%); brown solid; m. p. 142-144 ${ }^{\circ} \mathrm{C} ;[\alpha]_{\mathrm{D}}-23.5\left(c\right.$ 0.19, DMF); ${ }^{1} \mathrm{H}$ NMR (400 MHz, DMSO- $\left.d_{6}\right) \delta 1.18-1.30\left(\mathrm{~m}, 4 \mathrm{H}, \mathrm{CH}_{3}\right), 1.38$ 
(s, 8H, $\mathrm{CH}_{3}$ ), 1.89-2.07 (m, 2H, H-7), 2.19 (s, 3H, pyrrole- $\mathrm{CH}_{3}$ ), 2.66-2.77 (m, 3H, H-5, $\mathrm{H}_{\mathrm{A}}-4$ ), $3.01\left(\mathrm{dd}, 1 \mathrm{H}, J_{1}=15.9 \mathrm{~Hz}, J_{2}=5.1 \mathrm{~Hz}, \mathrm{H}_{\mathrm{B}}-4\right), 4.15-4.32(\mathrm{~m}, 2 \mathrm{H}, 2 \times \mathrm{C} \underline{\mathrm{HNH}}), 7.22(\mathrm{~d}, 1 \mathrm{H}, J=$

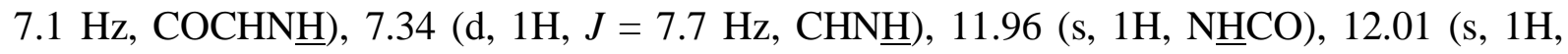
pyrrole-NH) ppm; ${ }^{13} \mathrm{C}$ NMR $\left(100 \mathrm{MHz}, \mathrm{DMSO}-d_{6}\right) \delta 11.1,18.1,24.7,28.5,28.7,28.8,45.6$, 50.1, 78.6, 108.5, 110.4, 119.7, 127.8, 143.9, 155.6, 156.0, 158.7, 172.3 ppm; HRMS ESI ${ }^{+} \mathrm{m} / \mathrm{z}$ for $\mathrm{C}_{21} \mathrm{H}_{28} \mathrm{~N}_{5} \mathrm{O}_{4} \mathrm{SCl}_{2}\left([\mathrm{M}+\mathrm{H}]^{+}\right)$: calcd 516.12336, found 516.12366; HPLC: $\mathrm{t}_{\mathrm{r}} 13.82$ min (96 \% at $254 \mathrm{~nm})$.

6.2.2.1.13 tert-Butyl ((S)-1-(((S)-6-(3,4-dichloro-5-methyl-1H-pyrrole-2-carboxamido)4,5,6,7-tetrahydrobenzo[d]thiazol-2-yl)amino)-3-methyl-1-oxobutan-2-yl)carbamate

(27). Prepared from $12(0.305 \mathrm{~g}, 0.883 \mathrm{mmol})$ and $N$-Boc-L-valine (0. $192 \mathrm{~g}, 0.883 \mathrm{mmol})$ according to the general procedure B. Product was purified by flash column chromatography using dichloromethane/methanol (20:1) as eluent. Yield 0.158 g (33 \%); brown solid; m. p. 144-146 ${ }^{\circ} \mathrm{C} ;[\alpha]_{\mathrm{D}}-10.2(c 0.20, \mathrm{DMF}) ;{ }^{1} \mathrm{H}$ NMR $\left(400 \mathrm{MHz}, \mathrm{DMSO}-d_{6}\right) \delta 0.80-0.95\left(\mathrm{~m}, 6 \mathrm{H}, 2 \times \mathrm{CHC}_{3}\right)$, 1.28 and $1.38\left(2 \times \mathrm{s}, 9 \mathrm{H}, 3 \times \mathrm{CH}_{3}\right), 1.89-2.06\left(\mathrm{~m}, 3 \mathrm{H}, \mathrm{H}-7, \mathrm{CHCH}_{3}\right), 2.19\left(\mathrm{~s}, 3 \mathrm{H}\right.$, pyrrole- $\left.\mathrm{CH}_{3}\right)$, 2.66-2.77 (m, 3H, H-5, $\left.\mathrm{H}_{\mathrm{A}}-4\right), 2.96-3.06\left(\mathrm{~m}, 1 \mathrm{H}, \mathrm{H}_{\mathrm{B}}-4\right), 4.01(\mathrm{t}, 1 \mathrm{H}, J=8.0 \mathrm{~Hz}, \mathrm{COC} \underline{\mathrm{HNH}})$, 4.21-4.32 (m, 1H, CㅍNH), $7.07(\mathrm{~d}, 1 \mathrm{H}, J=8.1 \mathrm{~Hz}, \mathrm{COCHN} \underline{\mathrm{H}}), 7.35(\mathrm{~d}, 1 \mathrm{H}, J=7.5 \mathrm{~Hz}$, CHN $\underline{H}), 11.98$ (s, 1H, N $\underline{H C O}), 12.01$ (s, 1H, pyrrole-NH) ppm; ${ }^{13} \mathrm{C}$ NMR (100 MHz, DMSO$\left.d_{6}\right) \delta 11.1,19.0,19.5,24.7,28.5,28.6,28.8,30.5,45.6,60.3,78.7,108.5,110.4,119.7,127.8$, 143.9, 156.0, 158.7, 171.2 ppm; HRMS $\mathrm{ESI}^{+} \mathrm{m} / \mathrm{z}$ for $\mathrm{C}_{23} \mathrm{H}_{32} \mathrm{~N}_{5} \mathrm{O}_{4} \mathrm{SCl}_{2}\left([\mathrm{M}+\mathrm{H}]^{+}\right)$: calcd 544.15466, found 544.15472; HPLC: $\mathrm{t}_{\mathrm{r}} 15.10 \mathrm{~min}(96 \%$ at $254 \mathrm{~nm})$.

6.2.2.1.14 tert-Butyl (S)-(3-((6-(3,4-dichloro-5-methyl-1H-pyrrole-2-carboxamido)-4,5,6,7tetrahydrobenzo[d]thiazol-2-yl)amino)-3-oxopropyl)carbamate (28). Prepared from 12 (0.339 $\mathrm{g}, 0.982 \mathrm{mmol})$ and $N$-Boc- $\beta$-alanine $(0.186 \mathrm{~g}, 0.982 \mathrm{mmol})$ according to the general procedure B. Yield $0.361 \mathrm{~g}(71 \%)$; brown solid; m. p. $167-169{ }^{\circ} \mathrm{C} ;[\alpha]_{\mathrm{D}}-6.8\left(c 0.32\right.$, DMF); ${ }^{1} \mathrm{H}$ NMR $\left(400 \mathrm{MHz}, \mathrm{DMSO}-d_{6}\right) \delta 1.37\left(\mathrm{~s}, 9 \mathrm{H}, 3 \times \mathrm{CH}_{3}\right), 1.87-2.07$ (m, 2H, H-7), 2.19 (s, 3H, pyrrole- 
$\left.\mathrm{CH}_{3}\right), 2.52-2.58\left(\mathrm{~m}, 2 \mathrm{H}, \mathrm{COCH}_{2}\right), 2.65-2.73\left(\mathrm{~m}, 3 \mathrm{H}, \mathrm{H}-5, \mathrm{H}_{\mathrm{A}}-4\right), 2.94-3.06\left(\mathrm{~m}, 1 \mathrm{H}, \mathrm{H}_{\mathrm{B}}-4\right), 3.22$ $\left(\mathrm{dd}, 2 \mathrm{H}, J_{1}=12.9 \mathrm{~Hz}, J_{2}=6.8 \mathrm{~Hz}, \mathrm{C}_{2} \mathrm{NH}\right), 4.18-4.32(\mathrm{~m}, 1 \mathrm{H}, \mathrm{C} \underline{\mathrm{HNH}}), 6.89(\mathrm{t}, 1 \mathrm{H}, J=5.5$

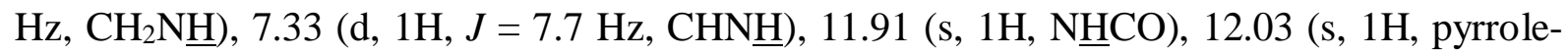
NH) ppm; ${ }^{13} \mathrm{C}$ NMR $\left(100 \mathrm{MHz}, \mathrm{DMSO}-d_{6}\right) \delta 11.1,24.7,28.6,28.7,28.8,35.9,36.6,45.7,78.1$, $108.4,110.5,119.4,119.7,127.8,143.7,155.9,156.0,158.7,169.7$ ppm; HRMS ESI ${ }^{+} \mathrm{m} / \mathrm{z}$ for $\mathrm{C}_{21} \mathrm{H}_{28} \mathrm{~N}_{5} \mathrm{O}_{4} \mathrm{SCl}_{2}\left([\mathrm{M}+\mathrm{H}]^{+}\right)$: calcd 516.12336, found 516.12366; HPLC: $\mathrm{t}_{\mathrm{r}} 13.44 \min (95 \%$ at $254 \mathrm{~nm})$.

6.2.2.1.15

(S)-2-((6-(4,5-Dibromo-1H-pyrrole-2-carboxamido)-4,5,6,7tetrahydrobenzo[d]thiazol-2-yl)amino)-2-oxoethan-1-aminium chloride (29). To an ice-cold anhydrous methanol $(70 \mathrm{~mL})$ acetyl chloride $(1.2 \mathrm{~mL}, 12.0 \mathrm{mmol})$ was added dropwise. Reaction mixture was stirred for $30 \mathrm{~min}$ at $0{ }^{\circ} \mathrm{C}$. Then a solution of $\mathbf{2 4}(0.691 \mathrm{~g}, 1.20 \mathrm{mmol})$ in anhydrous methanol $(40 \mathrm{~mL})$ was added and reaction mixture was stirred for $1 \mathrm{~h}$ at $0{ }^{\circ} \mathrm{C}$ and then for $18 \mathrm{~h}$ at room temperature. Solvent was evaporated under reduced pressure. Yield 0.493 g (80 \%); white solid; m. p. $197-199{ }^{\circ} \mathrm{C} ;[\alpha]_{\mathrm{D}}-4.42\left(c\right.$ 0.24, MeOH); ${ }^{1} \mathrm{H}$ NMR $(400 \mathrm{MHz}$, DMSO- $\left.d_{6}\right) \delta 1.81-1.93\left(\mathrm{~m}, 1 \mathrm{H}, \mathrm{H}_{\mathrm{A}}-7\right), 1.96-2.04\left(\mathrm{~m}, 1 \mathrm{H}, \mathrm{H}_{\mathrm{B}}-7\right), 2.63-2.74\left(\mathrm{~m}, 3 \mathrm{H}, \mathrm{H}-5, \mathrm{H}_{\mathrm{A}}-4\right)$, $2.98\left(\mathrm{dd}, 1 \mathrm{H}, J_{1}=15.5 \mathrm{~Hz}, J_{2}=5.4 \mathrm{~Hz}, \mathrm{H}_{\mathrm{B}}-4\right), 3.86\left(\mathrm{dd}, 2 \mathrm{H}, J_{1}=8.6 \mathrm{~Hz}, J_{2}=3.3 \mathrm{~Hz}, \underline{\mathrm{C}}_{2} \mathrm{NH}_{3}^{+}\right)$, 4.14-4.24 (m, 1H, CㅌNH), $7.02(\mathrm{~d}, 1 \mathrm{H}, J=2.8 \mathrm{~Hz}$, pyrrole-H), $8.23(\mathrm{~d}, 1 \mathrm{H}, J=7.8 \mathrm{~Hz}, \mathrm{CHN} \underline{\mathrm{H}})$, $8.31\left(\right.$ br s, $\left.3 \mathrm{H}, \mathrm{NH}_{3}{ }^{+}\right), 12.77\left(\mathrm{~d}, 1 \mathrm{H}, J=2.7 \mathrm{~Hz}\right.$, pyrrole-NH) ppm; ${ }^{13} \mathrm{C}$ NMR $(100 \mathrm{MHz}, \mathrm{DMSO}-$ $\left.d_{6}\right) \delta 24.6,28.4,28.6,40.6,45.2,97.8,104.5,113.1,120.0,128.0,142.0,157.3,157.7,158.3$ ppm; HRMS ESI' $\mathrm{m} / \mathrm{z}$ for $\mathrm{C}_{14} \mathrm{H}_{14} \mathrm{Br}_{2} \mathrm{~N}_{5} \mathrm{O}_{2} \mathrm{~S}$ ([M-H] $\left.]^{-}\right)$: calcd 473.9235, found 473.9230; HPLC: $\mathrm{t}_{\mathrm{r}} 10.80 \min (100 \%$ at $254 \mathrm{~nm})$.

6.2.2.2. General procedure C. Starting compound (1 mmol) was dissolved in anhydrous 1,4dioxane $(10 \mathrm{~mL})$ under argon atmosphere and cooled on an ice bath. Then a solution of $4 \mathrm{M}$ $\mathrm{HCl}$ in 1,4-dioxane $(2.5 \mathrm{~mL}, 10 \mathrm{mmol})$ was added dropwise and the mixture was stirred 
overnight at room temperature. After reaction was completed solvent was evaporated under reduced pressure and co-evaporation with diethyl ether was performed $(2 \times 10 \mathrm{~mL})$.

6.2.2.2.1 (S)-2-((6-(3,4-Dichloro-5-methyl-1H-pyrrole-2-carboxamido)-4,5,6,7tetrahydrobenzo[d]thiazol-2-yl)amino)-2-oxoethan-1-aminium chloride (30). Prepared from 25 $(0.133 \mathrm{~g}, 0.265 \mathrm{mmol})$ according to the general procedure C. Yield $0.116 \mathrm{~g}$ (100\%); grey amorphous solid; $[\alpha]_{\mathrm{D}}-6.2\left(c\right.$ 0.24, DMF); ${ }^{1} \mathrm{H}$ NMR $\left(400 \mathrm{MHz}, \mathrm{DMSO}-d_{6}\right) \delta 1.86-2.08(\mathrm{~m}, 2 \mathrm{H}$, H-7), 2.18 (s, 3H, pyrrole- $\left.\mathrm{CH}_{3}\right), 2.66-2.80\left(\mathrm{~m}, 3 \mathrm{H}, \mathrm{H}-5, \mathrm{H}_{\mathrm{A}}-4\right), 3.02\left(\mathrm{dd}, 1 \mathrm{H}, J_{1}=15.9 \mathrm{~Hz}, J_{2}\right.$ $\left.=5.2 \mathrm{~Hz}, \mathrm{H}_{\mathrm{B}}-4\right), 3.83-3.91\left(\mathrm{~m}, 2 \mathrm{H}, \mathrm{C}_{2} \mathrm{NH}_{3}{ }^{+}\right), 4.18-4.29(\mathrm{~m}, 1 \mathrm{H}, \mathrm{C} \underline{\mathrm{HNH}}), 7.72(\mathrm{~d}, 1 \mathrm{H}, J=7.6$ $\mathrm{Hz}, \mathrm{CHN} \underline{\mathrm{H}}), 8.42$ (br s, $\left.3 \mathrm{H}, \mathrm{NH}_{3}{ }^{+}\right), 12.38\left(\mathrm{~s}, 1 \mathrm{H}\right.$, pyrrole-NH) ppm; ${ }^{13} \mathrm{C}$ NMR $(100 \mathrm{MHz}$, DMSO- $\left.d_{6}\right) \delta 10.5,24.1,28.0,28.2,40.5,45.0,107.9,110.4,119.0,119.8,127.1,158.1,164.9$ ppm; HRMS ESI ${ }^{+} \mathrm{m} / \mathrm{z}$ for $\mathrm{C}_{15} \mathrm{H}_{18} \mathrm{~N}_{5} \mathrm{O}_{2} \mathrm{SCl}_{2}\left([\mathrm{M}+\mathrm{H}]^{+}\right)$: calcd 402.0564, found 402.0555; HPLC: $\mathrm{t}_{\mathrm{r}} 9.33 \min (95 \%$ at $254 \mathrm{~nm})$.

(S)-1-(((S)-6-(3,4-Dichloro-5-methyl-1H-pyrrole-2-carboxamido)-4,5,6,7tetrahydrobenzo[d]thiazol-2-yl)amino)-1-oxopropan-2-aminium chloride (31). Prepared from 26 (0.186 g, $0.360 \mathrm{mmol})$ according to the general procedure C. Yield $0.120 \mathrm{~g}$ (74 \%); brown solid; m. p. $177-179{ }^{\circ} \mathrm{C} ;[\alpha]_{\mathrm{D}}-12.6\left(c\right.$ 0.24, DMF); ${ }^{1} \mathrm{H}$ NMR (400 MHz, DMSO- $\left.d_{6}\right) \delta 1.46(\mathrm{~d}$, $\left.3 \mathrm{H}, J=7.0 \mathrm{~Hz}, \mathrm{CH}_{3}\right), 1.89-2.08(\mathrm{~m}, 2 \mathrm{H}, \mathrm{H}-7), 2.19\left(\mathrm{~s}, 3 \mathrm{H}\right.$, pyrrole- $\left.\mathrm{CH}_{3}\right), 2.70-2.81(\mathrm{~m}, 3 \mathrm{H}, \mathrm{H}-$ 5, $\left.\mathrm{H}_{\mathrm{A}}-4\right), 3.03\left(\mathrm{dd}, 1 \mathrm{H}, J_{1}=15.5 \mathrm{~Hz}, J_{2}=4.8 \mathrm{~Hz}, \mathrm{H}_{\mathrm{B}}-4\right), 4.05-4.16\left(\mathrm{~m}, 1 \mathrm{H}, \mathrm{CHNH}_{3}{ }^{+}\right), 4.18-$ $4.30(\mathrm{~m}, 1 \mathrm{H}, \mathrm{C} \underline{\mathrm{HNH}}), 7.61(\mathrm{~d}, 1 \mathrm{H}, J=7.7 \mathrm{~Hz}, \mathrm{CHN} \underline{\mathrm{H}}), 8.46\left(\mathrm{br} \mathrm{s}, 3 \mathrm{H}, \mathrm{NH}_{3}{ }^{+}\right), 12.28(\mathrm{~s}, 1 \mathrm{H}$, pyrrole-NH) ppm; HRMS ESI ${ }^{+} \mathrm{m} / \mathrm{z}$ for $\mathrm{C}_{16} \mathrm{H}_{20} \mathrm{~N}_{5} \mathrm{O}_{2} \mathrm{SCl}_{2}\left([\mathrm{M}+\mathrm{H}]^{+}\right)$: calcd 416.07093, found 416.07080; HPLC: $\mathrm{t}_{\mathrm{r}} 9.48 \mathrm{~min}(96 \%$ at $254 \mathrm{~nm})$.

6.2.2.2.3 (S)-1-(((S)-6-(3,4-Dichloro-5-methyl-1H-pyrrole-2-carboxamido)-4,5,6,7tetrahydrobenzo[d]thiazol-2-yl)amino)-3-methyl-1-oxobutan-2-aminium chloride (32). Prepared from $27(0.136 \mathrm{~g}, 0.249 \mathrm{mmol})$ according to the general procedure $\mathbf{C}$. Yield $0.120 \mathrm{~g}$ 
(100\%); brown solid; m. p. 200-202 ${ }^{\circ} \mathrm{C} ;[\alpha]_{\mathrm{D}}+25.6$ (c 0.16, DMF); ${ }^{1} \mathrm{H}$ NMR $(400 \mathrm{MHz}$, DMSO- $\left.d_{6}\right) \delta 0.97\left(\mathrm{dd}, 6 \mathrm{H}, J_{1}=6.8 \mathrm{~Hz}, J_{2}=1.1 \mathrm{~Hz}, 2 \times \mathrm{CHC}_{3}\right), 1.89-2.08(\mathrm{~m}, 3 \mathrm{H}, \mathrm{H}-7$, $\left.\mathrm{CHCH}_{3}\right), 2.19$ (s, 3H, pyrrole- $\left.\mathrm{CH}_{3}\right), 2.69-2.82\left(\mathrm{~m}, 3 \mathrm{H}, \mathrm{H}-5, \mathrm{H}_{\mathrm{A}}-4\right), 2.97-3.08\left(\mathrm{~m}, 1 \mathrm{H}, \mathrm{H}_{\mathrm{B}}-4\right)$,

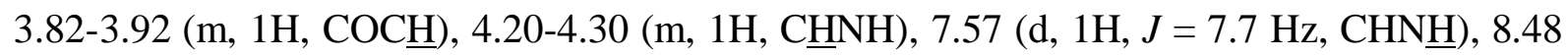
(br s, $3 \mathrm{H}, \mathrm{NH}_{3}{ }^{+}$), 12.24 (s, $1 \mathrm{H}$, pyrrole-NH) ppm; HRMS $\mathrm{ESI}^{+} \mathrm{m} / \mathrm{z}$ for $\mathrm{C}_{18} \mathrm{H}_{24} \mathrm{~N}_{5} \mathrm{O}_{2} \mathrm{SCl}_{2}$ $\left([\mathrm{M}+\mathrm{H}]^{+}\right)$: calcd 444.10223, found 444.10208; HPLC: $\mathrm{t}_{\mathrm{r}} 9.91 \mathrm{~min}(95 \%$ at $254 \mathrm{~nm})$.

6.2.2.2.4

(S)-3-((6-(3,4-Dichloro-5-methyl-1H-pyrrole-2-carboxamido)-4,5,6,7tetrahydrobenzo[d]thiazol-2-yl)amino)-3-oxopropan-1-aminium chloride (33). Prepared from $28(0.247 \mathrm{~g}, 0.478 \mathrm{mmol})$ according to the general procedure C. Yield $0.181 \mathrm{~g}(83 \%)$; grey solid; m. p. $210-212{ }^{\circ} \mathrm{C} ;[\alpha]_{\mathrm{D}}-16.6$ (c 0.24, DMF); ${ }^{1} \mathrm{H}$ NMR (400 MHz, DMSO- $\left.d_{6}\right) \delta 1.87-2.07$ (m, 2H, H-7), 2.19 (s, 3H, pyrrole- $\left.\mathrm{CH}_{3}\right), 2.66-2.79\left(\mathrm{~m}, 3 \mathrm{H}, \mathrm{H}-5, \mathrm{H}_{\mathrm{A}}-4\right), 2.82(\mathrm{t}, 2 \mathrm{H}, J=6.9 \mathrm{~Hz}$, $\left.\mathrm{COCH}_{2}\right), 2.96-3.05\left(\mathrm{~m}, 1 \mathrm{H}, \mathrm{H}_{\mathrm{B}}-4\right), 3.05-3.14\left(\mathrm{~m}, 2 \mathrm{H}, \mathrm{C}_{2} \mathrm{NH}_{3}{ }^{+}\right), 4.19-4.30(\mathrm{~m}, 1 \mathrm{H}, \mathrm{C} \underline{\mathrm{HNH}})$, $7.57(\mathrm{~d}, 1 \mathrm{H}, J=7.8 \mathrm{~Hz}, \mathrm{CHN} \underline{\mathrm{H}}), 7.97$ (br s, $3 \mathrm{H}, \mathrm{NH}_{3}{ }^{+}$), 12.17 (br s, $1 \mathrm{H}, \mathrm{N} \underline{\mathrm{HCO}}$ ), 12.25 (s, 1H, pyrrole-NH) ppm; ${ }^{13} \mathrm{C}$ NMR (100 MHz, DMSO- $\left.d_{6}\right) \delta 11.1,24.8,28.6,28.8,32.8,34.8,45.7$, 108.5, 111.1, 119.5, 119.8, 127.5, 143.7, 155.7, 158.6, $168.7 \mathrm{ppm} ; \mathrm{HRMS} \mathrm{ESI}^{+} \mathrm{m} / \mathrm{z}$ for $\mathrm{C}_{16} \mathrm{H}_{20} \mathrm{~N}_{5} \mathrm{O}_{2} \mathrm{SCl}_{2}\left([\mathrm{M}+\mathrm{H}]^{+}\right)$: calcd 416.07093, found 416.07077; HPLC: $\mathrm{t}_{\mathrm{r}} 9.46 \min (97 \%$ at $254 \mathrm{~nm})$.

6.2.2.3.1. (S)-3,4-Dichloro-N-(2-(2-(3,4-dihydroxybenzamido)acetamido)-4,5,6,7tetrahydrobenzo[d]thiazol-6-yl)-5-methyl-1H-pyrrole-2-carboxamide (34). Prepared from 30 $(0.142 \mathrm{~g}, 0.324 \mathrm{mmol})$ and 3,4-dihydroxybenzoic acid $(0.050 \mathrm{~g}, 0.324 \mathrm{mmol})$ according to the general procedure B. Yield $0.089 \mathrm{~g}(51 \%)$; brown solid; m. p. 203-205 ${ }^{\circ} \mathrm{C} ;[\alpha]_{\mathrm{D}}+4.3(c 0.28$, DMF); ${ }^{1} \mathrm{H}$ NMR (400 MHz, DMSO- $\left.d_{6}\right) \delta$ 1.87-2.06 (m, 2H, H-7), 2.18 (s, 3H, pyrrole- $\mathrm{CH}_{3}$ ), 2.65-2.77 (m, 3H, H-5, $\left.\mathrm{H}_{\mathrm{A}}-4\right), 3.01\left(\mathrm{dd}, 1 \mathrm{H}, J_{1}=15.7 \mathrm{~Hz}, J_{2}=5.2 \mathrm{~Hz}, \mathrm{H}_{\mathrm{B}}-4\right), 4.07(\mathrm{~d}, 2 \mathrm{H}, J=$ $\left.6.5 \mathrm{~Hz}, \mathrm{CH}_{2} \mathrm{NH}\right), 4.20-4.32(\mathrm{~m}, 1 \mathrm{H}, \mathrm{CHNH}), 6.78(\mathrm{~d}, 1 \mathrm{H}, J=8.2 \mathrm{~Hz}, \mathrm{CHNH}), 7.23$ (dd, 1H, $J_{1}$ $\left.=8.3 \mathrm{~Hz}, J_{2}=2.2 \mathrm{~Hz}, \mathrm{Ar}-\mathrm{H}\right), 7.28-7.35(\mathrm{~m}, 2 \mathrm{H}, \mathrm{Ar}-\mathrm{H}), 8.52\left(\mathrm{t}, 1 \mathrm{H}, J=5.9 \mathrm{~Hz}, \mathrm{CH}_{2} \mathrm{NH}\right), 9.15$ 
(br s, 1H, OH), 9.50 (br s, 1H, OH), 12.01 (s, 2H, pyrrole-NH, N $\underline{H C O})$ ppm; ${ }^{13} \mathrm{C}$ NMR (100 MHz, DMSO- $\left.d_{6}\right) \delta 11.1,24.7,28.5,28.8,43.0,45.6,108.5,110.4,115.3,115.6,119.6,119.7$, 125.4, 127.8, 143.8, 145.3, 149.1, 155.9, 158.7, 167.0, $168.6 \mathrm{ppm} ; \mathrm{HRMS} \mathrm{ESI}^{-} \mathrm{m} / \mathrm{z}$ for $\mathrm{C}_{22} \mathrm{H}_{20} \mathrm{~N}_{5} \mathrm{O}_{5} \mathrm{SCl}_{2}\left([\mathrm{M}-\mathrm{H}]^{-}\right)$: calcd 536.0562, found 536.0555; HPLC: $\mathrm{t}_{\mathrm{r}} 10.89 \min (95 \%$ at 254 $\mathrm{nm})$.

6.2.2.3.2 (S)-3,4-Dichloro-N-(2-(2-(2,3-dihydroxybenzamido)acetamido)-4,5,6,7tetrahydrobenzo[d]thiazol-6-yl)-5-methyl-1H-pyrrole-2-carboxamide (35). Prepared from 30 $(0.142 \mathrm{~g}, 0.324 \mathrm{mmol})$ and 2,3-dihydroxybenzoic acid $(0.050 \mathrm{~g}, 0.324 \mathrm{mmol})$ according to the general procedure B. Yield $0.043 \mathrm{~g}(25 \%)$; yellow solid; m. p. $173-175{ }^{\circ} \mathrm{C} ;[\alpha]_{\mathrm{D}}+29.8(c 0.20$, DMF); ${ }^{1} \mathrm{H}$ NMR (400 MHz, DMSO-d $) \delta$ 1.89-2.06 (m, 2H, H-7), 2.18 (s, 3H, pyrrole- $\mathrm{CH}_{3}$ ), 2.55-2.73 (m, 3H, H-5, $\left.\mathrm{H}_{\mathrm{A}}-4\right), 3.01\left(\mathrm{dd}, 1 \mathrm{H}, J_{1}=15.7 \mathrm{~Hz}, J_{2}=5.2 \mathrm{~Hz}, \mathrm{H}_{\mathrm{B}}-4\right), 4.18(\mathrm{~d}, 2 \mathrm{H}, J=$ $\left.5.7 \mathrm{~Hz}, \underline{\mathrm{C}}_{2} \mathrm{NH}\right), 4.21-4.30$ (m, 1H, $\left.\mathrm{C} \underline{\mathrm{HNH}}\right), 6.69$ (t, $\left.1 \mathrm{H}, J=7.7 \mathrm{~Hz}, \mathrm{CH}_{2} \mathrm{NH}\right), 6.93(\mathrm{~d}, 1 \mathrm{H}, J$ $=6.9 \mathrm{~Hz}, \mathrm{Ar}-\mathrm{H}), 7.29-7.36(\mathrm{~m}, 2 \mathrm{H}, \mathrm{Ar}-\mathrm{H}), 9.31(\mathrm{br} \mathrm{s}, 1 \mathrm{H}, \mathrm{OH}), 11.78-12.30(\mathrm{~m}, 2 \mathrm{H}$, pyrrole$\mathrm{NH}, \mathrm{N} \underline{\mathrm{HCO}}) \mathrm{ppm} ;{ }^{13} \mathrm{C}$ NMR $\left(100 \mathrm{MHz}, \mathrm{DMSO}-d_{6}\right) \delta 11.1,24.7,28.5,28.8,42.7,45.6,108.5$, 110.4, 115.5, 118.3, 119.2, 119.7, 119.8, 127.8, 146.7, 155.8, 158.7, 167.9, 170.3 ppm; HRMS $\mathrm{ESI}^{-} \mathrm{m} / \mathrm{z}$ for $\mathrm{C}_{22} \mathrm{H}_{20} \mathrm{~N}_{5} \mathrm{O}_{5} \mathrm{SCl}_{2}\left([\mathrm{M}-\mathrm{H}]^{-}\right)$: calcd 536.0562, found 536.0556; HPLC: $\mathrm{t}_{\mathrm{r}} 12.00 \mathrm{~min}$ (95\% at $254 \mathrm{~nm})$.

6.2.2.3.3 (S)-N-(2-(2-(5-(Benzyloxy)-4-oxo-4H-pyran-2-carboxamido)acetamido)-4,5,6,7tetrahydrobenzo[d]thiazol-6-yl)-4,5-dibromo-1H-pyrrole-2-carboxamide (36). Prepared from $29(0.150 \mathrm{~g}, 0.292 \mathrm{mmol})$ and $7(0.072 \mathrm{~g}, 0.292 \mathrm{mmol})$ according to the general procedure $\mathbf{B}$. Crude product was crystallized from dichloromethane/methanol (20:1). Yield 0.028 g (14 \%); yellow solid; m. p. $166-168{ }^{\circ} \mathrm{C} ;[\alpha]_{\mathrm{D}}+18.4$ (c 0.08, DMF); ${ }^{1} \mathrm{H}$ NMR (400 MHz, DMSO- $\left.d_{6}\right) \delta$ 1.81-1.92 (m, 1H, $\left.\mathrm{H}_{\mathrm{A}}-7\right), 1.95-2.05\left(\mathrm{~m}, 1 \mathrm{H}, \mathrm{H}_{\mathrm{B}}-7\right), 2.65-2.76\left(\mathrm{~m}, 3 \mathrm{H}, \mathrm{H}-5, \mathrm{H}_{\mathrm{A}}-4\right), 2.98(\mathrm{dd}, 1 \mathrm{H}$, $\left.J_{1}=15.7 \mathrm{~Hz}, J_{2}=4.5 \mathrm{~Hz}, \mathrm{H}_{\mathrm{B}}-4\right), 4.13\left(\mathrm{~d}, 2 \mathrm{H}, J=6.0 \mathrm{~Hz}, \mathrm{CH}_{2} \mathrm{NH}\right), 4.16-4.23(\mathrm{~m}, 1 \mathrm{H}, \mathrm{CHNH})$, $5.03\left(\mathrm{~s}, 2 \mathrm{H}, \mathrm{OCH}_{2}\right), 6.91(\mathrm{~s}, 1 \mathrm{H}$, pyran-H), $7.01(\mathrm{~s}, 1 \mathrm{H}$, pyrrole-H), 7.35-7.47 (m, 5H, Ph-H), 
$8.18(\mathrm{~d}, 1 \mathrm{H}, J=8.4 \mathrm{~Hz}, \mathrm{CHN} \underline{\mathrm{H}}), 8.32\left(\mathrm{~s}, 1 \mathrm{H}\right.$, pyran-H), $9.32\left(\mathrm{t}, 1 \mathrm{H}, J=6.0 \mathrm{~Hz}, \mathrm{CH}_{2} \mathrm{~N} \underline{\mathrm{H}}\right), 12.13$ (s, 1H, N $\underline{H C O}$ ), 12.74 (s, 1H, pyrrole-NH) ppm; HRMS ESI ${ }^{-} \mathrm{m} / \mathrm{z}$ for $\mathrm{C}_{27} \mathrm{H}_{22} \mathrm{Br}_{2} \mathrm{~N}_{5} \mathrm{O}_{6} \mathrm{~S}$ ([M-H] $]^{-}$ ): calcd 701.9658, found 701.9658; HPLC: $t_{\mathrm{r}} 12.39 \min (95 \%$ at $254 \mathrm{~nm})$.

\subsubsection{4 (S)-4,5-Dibromo-N-(2-(2-(5-hydroxy-4-oxo-4H-pyran-2-carboxamido)acetamido)-} 4,5,6,7-tetrahydrobenzo[d]thiazol-6-yl)-1H-pyrrole-2-carboxamide (37). Prepared from 29 $(0.100 \mathrm{~g}, 0.195 \mathrm{mmol})$ and $\mathbf{9}(0.030 \mathrm{~g}, 0.195 \mathrm{mmol})$ according to the general procedure $\mathbf{B}$. Crude product was purified by flash column chromatography using dichloromethane/methanol (9:1) to dichloromethane/methanol (4:1) as eluent. Yield $0.008 \mathrm{~g} \mathrm{(12 \% );} \mathrm{yellow} \mathrm{amorphous} \mathrm{solid;}$ ${ }^{1} \mathrm{H}$ NMR $\left(400 \mathrm{MHz}, \mathrm{DMSO}-d_{6}\right) \delta 1.83-1.91\left(\mathrm{~m}, 1 \mathrm{H}, \mathrm{H}_{\mathrm{A}}-7\right), 1.95-2.03\left(\mathrm{~m}, 1 \mathrm{H}, \mathrm{H}_{\mathrm{B}}-7\right), 2.65-2.73$ $\left(\mathrm{m}, 3 \mathrm{H}, \mathrm{H}-5, \mathrm{H}_{\mathrm{A}}-4\right), 2.98\left(\mathrm{dd}, 1 \mathrm{H}, J_{1}=15.6 \mathrm{~Hz}, J_{2}=5.2 \mathrm{~Hz}, \mathrm{H}_{\mathrm{B}}-4\right), 4.13(\mathrm{~d}, 2 \mathrm{H}, J=5.8 \mathrm{~Hz}$, $\left.\mathrm{C}_{2} \mathrm{NH}\right), 4.15-4.21(\mathrm{~m}, 1 \mathrm{H}, \mathrm{C} \underline{\mathrm{HNH}}), 6.92(\mathrm{~s}, 1 \mathrm{H}$, pyran-H), 7.00 (s, 1H, pyrrole-H), 8.18-8.23

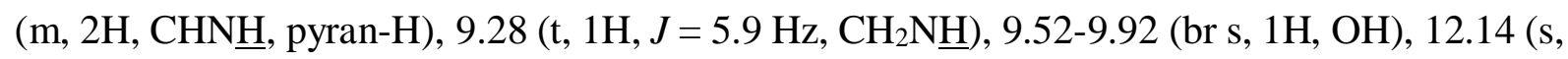
$1 \mathrm{H}$, pyrrole-NH), 12.62-12.96 (br s, $1 \mathrm{H}, \mathrm{NHCO}$ ) ppm; HRMS ESI- m/z for $\mathrm{C}_{20} \mathrm{H}_{16} \mathrm{Br}_{2} \mathrm{~N}_{5} \mathrm{O}_{6} \mathrm{~S}$ ([M-H] $\left.]^{-}\right)$: calcd 611.9188, found 611.9180.

\subsubsection{5 (S)-N-(2-(2-(5-(Benzyloxy)-4-oxo-4H-pyran-2-carboxamido)acetamido)-4,5,6,7-} tetrahydrobenzo[d]thiazol-6-yl)-3,4-dichloro-5-methyl-1H-pyrrole-2-carboxamide

(38). Prepared from $30(0.125 \mathrm{~g}, 0.287 \mathrm{mmol})$ and $7(0.071 \mathrm{~g}, 0.287 \mathrm{mmol})$ according to the general procedure B. Crude product was purified by crystallization from methanol. Yield $0.045 \mathrm{~g}$ (25 \%); yellow solid; m. p. $183-185{ }^{\circ} \mathrm{C} ;[\alpha]_{\mathrm{D}}-10.9$ (c 0.11, DMF); ${ }^{1} \mathrm{H}$ NMR (400 MHz, DMSO- $\left.d_{6}\right)$ $\delta$ 1.91-2.05 (m, 2H, H-7), 2.19 (s, 3H, pyrrole- $\left.\mathrm{CH}_{3}\right), 2.70-2.78\left(\mathrm{~m}, 3 \mathrm{H}, \mathrm{H}-5, \mathrm{H}_{\mathrm{A}}-4\right), 2.99-3.05$ (m, 1H, $\left.\mathrm{H}_{\mathrm{B}}-4\right), 4.13\left(\mathrm{~d}, 2 \mathrm{H}, J=6.0 \mathrm{~Hz}, \underline{\mathrm{C}}_{2} \mathrm{NH}\right), 4.22-4.31(\mathrm{~m}, 1 \mathrm{H}, \mathrm{C} \underline{\mathrm{HNH}}), 5.03(\mathrm{~s}, 2 \mathrm{H}$, $\left.\mathrm{OCH}_{2}\right), 6.91(\mathrm{~s}, 1 \mathrm{H}$, pyran-H), $7.33(\mathrm{~d}, 1 \mathrm{H}, J=7.6 \mathrm{~Hz}, \mathrm{CHN} \underline{\mathrm{H}}), 7.36-7.47$ (m, 5H, Ph-H), 8.32 (s, 1H, pyran-H), $9.31\left(\mathrm{t}, 1 \mathrm{H}, J=6.0 \mathrm{~Hz}, \mathrm{CH}_{2} \mathrm{~N} \underline{\mathrm{H}}\right), 12.02$ (s, 1H, pyrrole-NH), 12.13 (s, 1H, NHCO) ppm; HRMS ESI' $\mathrm{m} / \mathrm{z}$ for $\mathrm{C}_{28} \mathrm{H}_{24} \mathrm{~N}_{5} \mathrm{O}_{6} \mathrm{SCl}_{2}\left([\mathrm{M}-\mathrm{H}]^{-}\right)$: calcd 628.0824, found 628.0812; HPLC: $\mathrm{t}_{\mathrm{r}} 12.82 \mathrm{~min}(95 \%$ at $254 \mathrm{~nm})$. 
6.2.2.3.6 (S)-3,4-Dichloro-N-(2-(2-(5-hydroxy-4-oxo-4H-pyran-2-carboxamido)acetamido)4,5,6,7-tetrahydrobenzo[d]thiazol-6-yl)-5-methyl-1H-pyrrole-2-carboxamide (39). Prepared from $30(0.100 \mathrm{~g}, 0.228 \mathrm{mmol})$ and $\mathbf{9}(0.036 \mathrm{~g}, 0.228 \mathrm{mmol})$ according to the general procedure B. Product was precipitated from the reaction mixture after addition of $10 \%$ citric acid $(5 \mathrm{~mL})$ and was filtered off. Precipitate was then washed with hot ethyl acetate $(3 \times 5 \mathrm{~mL})$ and hot methanol $(3 \times 5 \mathrm{~mL})$ and dried. Yield $0.030 \mathrm{~g}(24 \%)$; brown amorphous solid; $[\alpha]_{\mathrm{D}}+10.9(c$ 0.17, DMF); ${ }^{1} \mathrm{H}$ NMR (400 MHz, DMSO-d6) $\delta$ 1.88-2.07 (m, 2H, H-7), 2.18 (s, 3H, pyrrole$\left.\mathrm{CH}_{3}\right), 2.65-2.77\left(\mathrm{~m}, 3 \mathrm{H}, \mathrm{H}-5, \mathrm{H}_{\mathrm{A}}-4\right), 3.02\left(\mathrm{dd}, 1 \mathrm{H}, J_{1}=15.8 \mathrm{~Hz}, J_{2}=5.1 \mathrm{~Hz}, \mathrm{H}_{\mathrm{B}}-4\right), 4.12(\mathrm{~d}$, $\left.2 \mathrm{H}, J=5.8 \mathrm{~Hz}, \underline{\mathrm{C}}_{2} \mathrm{NH}\right), 4.20-4.32(\mathrm{~m}, 1 \mathrm{H}, \mathrm{C} \underline{\mathrm{HNH}}), 6.92(\mathrm{~s}, 1 \mathrm{H}$, pyran-H), $7.33(\mathrm{~d}, 1 \mathrm{H}, J=$ $7.8 \mathrm{~Hz}, \mathrm{CHN} \underline{\mathrm{H}}), 8.19$ (s, 1H, pyran-H), 9.27 (t, 1H, J=5.8 Hz, $\left.\mathrm{CH}_{2} \mathrm{~N} \underline{\mathrm{H}}\right), 9.64$ (br s, $\left.1 \mathrm{H}, \mathrm{OH}\right)$, $12.02(\mathrm{~s}, 1 \mathrm{H}$, pyrrole-NH), 12.12 (s, $1 \mathrm{H}, \mathrm{NHCO}) \mathrm{ppm} ;{ }^{13} \mathrm{C}$ NMR (100 MHz, DMSO-d $) \delta 10.1$, 23.6, 27.4, 27.7, 41.7, 44.5, 107.4, 109.4, 112.6, 118.6, 118.8, 126.8, 138.8, 142.8, 146.9, 154.1, 154.7, 157.6, 158.6, 166.4, 173.1 ppm; HRMS ESI ${ }^{+} \mathrm{m} / \mathrm{z}$ for $\mathrm{C}_{21} \mathrm{H}_{20} \mathrm{~N}_{5} \mathrm{O}_{6} \mathrm{SCl}_{2}\left([\mathrm{M}+\mathrm{H}]^{+}\right)$: calcd 540.0517, found 540.0503; HPLC: $\mathrm{t}_{\mathrm{r}} 10.68 \mathrm{~min}(95 \%$ at $254 \mathrm{~nm})$.

6.2.2.3.7

(S)-N-(2-((6-(3,4-Dichloro-5-methyl-1H-pyrrole-2-carboxamido)-4,5,6,7tetrahydrobenzo[d]thiazol-2-yl)amino)-2-oxoethyl)-5-((4-methoxybenzyl)oxy)-4-oxo-1,4dihydropyridine-2-carboxamide (40). Prepared from $30(0.080 \mathrm{~g}, 0.182 \mathrm{mmol})$ and $\mathbf{1 0}(0.050$ $\mathrm{g}, 0.182 \mathrm{mmol})$ according to the general procedure B. Yield $0.053 \mathrm{~g}(45 \%)$; yellow solid; $\mathrm{m}$. p. $168-170{ }^{\circ} \mathrm{C} ;[\alpha]_{\mathrm{D}}+9.0\left(c\right.$ 0.16, DMF); ${ }^{1} \mathrm{H}$ NMR (400 MHz, DMSO- $\left.d_{6}\right) \delta 1.90-2.05(\mathrm{~m}, 2 \mathrm{H}$, $\mathrm{H}-7), 2.18\left(\mathrm{~s}, 3 \mathrm{H}\right.$, pyrrole- $\left.\mathrm{CH}_{3}\right), 2.65-2.75\left(\mathrm{~m}, 3 \mathrm{H}, \mathrm{H}-5, \mathrm{H}_{\mathrm{A}}-4\right), 3.02\left(\mathrm{dd}, 1 \mathrm{H}, J_{1}=15.8 \mathrm{~Hz}, J_{2}\right.$ $\left.=5.1 \mathrm{~Hz}, \mathrm{H}_{\mathrm{B}}-4\right), 3.76\left(\mathrm{~s}, 3 \mathrm{H}, \mathrm{OCH}_{3}\right), 4.15\left(\mathrm{~d}, 2 \mathrm{H}, J=6.0 \mathrm{~Hz}, \underline{\mathrm{C}}_{2} \mathrm{NH}\right), 4.22-4.32(\mathrm{~m}, 1 \mathrm{H}$,

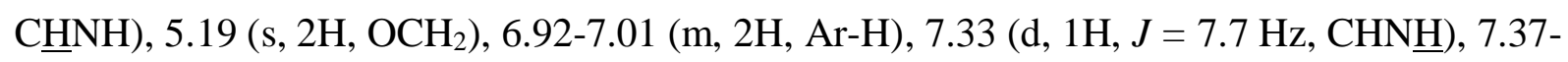
7.45 (m, 2H, Ar-H), 7.49 (s, 1H, pyridine-H), $8.23(\mathrm{~s}, 1 \mathrm{H}$, pyridine- $\mathrm{H}), 8.81(\mathrm{t}, 1 \mathrm{H}, J=6.0 \mathrm{~Hz}$, $\mathrm{CH}_{2} \mathrm{NH}$ ), 10.82 (br s, $1 \mathrm{H}$, pyridine- $\mathrm{NH}$ ), 12.02 (s, 1H, pyrrole-NH), 12.08 (br s, $1 \mathrm{H}, \mathrm{NHCO}$ ) ppm; ${ }^{13} \mathrm{C}$ NMR $\left(100 \mathrm{MHz}, \mathrm{DMSO}-d_{6}\right) \delta 11.2,28.5,28.8,55.5,70.8,108.4,110.4,114.3,119.7$, 
127.8, 128.9, 130.3, 158.7, 159.6, $168.1 \mathrm{ppm} ; \mathrm{HRMS} \mathrm{ESI}^{+} \mathrm{m} / \mathrm{z}$ for $\mathrm{C}_{29} \mathrm{H}_{29} \mathrm{~N}_{6} \mathrm{O}_{6} \mathrm{SCl}_{2}\left([\mathrm{M}+\mathrm{H}]^{+}\right)$: calcd 659.1252, found 659.1237; HPLC: $\mathrm{t}_{\mathrm{r}} 12.53 \mathrm{~min}(95 \%$ at $254 \mathrm{~nm})$.

6.2.2.3.8 N-((S)-1-(((S)-6-(3,4-Dichloro-5-methyl-1H-pyrrole-2-carboxamido)-4,5,6,7tetrahydrobenzo[d]thiazol-2-yl)amino)-1-oxopropan-2-yl)-5-((4-methoxybenzyl)oxy)-4-oxo1,4-dihydropyridine-2-carboxamide (41). Prepared from 31 (0.088 g, $0.194 \mathrm{mmol})$ and 10 (0.053 g, $0.194 \mathrm{mmol})$ according to the general procedure B. Product was purified by flash column chromatography using dichloromethane/methanol (20:1) as eluent. Yield $0.035 \mathrm{~g}$ (27 \%); brown solid; m. p. 147-149 ${ }^{\circ} \mathrm{C} ;[\alpha]_{\mathrm{D}}+15.7$ ( $c$ 0.04, DMF); ${ }^{1} \mathrm{H}$ NMR (400 MHz, DMSO- $\left.d_{6}\right)$ $\delta 1.43\left(\mathrm{~d}, 3 \mathrm{H}, J=7.1 \mathrm{~Hz}, \mathrm{CHC}_{3}\right), 1.89-2.06(\mathrm{~m}, 2 \mathrm{H}, \mathrm{H}-7), 2.19$ (s, 3H, pyrrole- $\left.\mathrm{CH}_{3}\right), 2.66-$ $2.78\left(\mathrm{~m}, 3 \mathrm{H}, \mathrm{H}-5, \mathrm{H}_{\mathrm{A}}-4\right), \quad 2.97-3.06\left(\mathrm{~m}, 1 \mathrm{H}, \mathrm{H}_{\mathrm{B}}-4\right), 3.76\left(\mathrm{~s}, 3 \mathrm{H}, \mathrm{OCH}_{3}\right), 4.21-4.32(\mathrm{~m}, 1 \mathrm{H}$, C$\underline{H N H}), 4.60-4.71\left(\mathrm{~m}, 1 \mathrm{H}, \mathrm{CHCH}_{3}\right), 5.19\left(\mathrm{~s}, 2 \mathrm{H}, \mathrm{OCH}_{2}\right), 6.93-6.99$ (m, 2H, Ar-H), 7.34 (d, $1 \mathrm{H}, J=7.8 \mathrm{~Hz}, \mathrm{CHN} \underline{\mathrm{H}}), 7.38-7.45(\mathrm{~m}, 2 \mathrm{H}, \mathrm{Ar}-\mathrm{H}), 7.48(\mathrm{~s}, 1 \mathrm{H}$, pyridine-H), $8.23(\mathrm{~s}, 1 \mathrm{H}$, pyridine-H), 8.60-8.67 (m, 1H, COCHN $\underline{H}), 10.87(\mathrm{~s}, 1 \mathrm{H}$, pyridine-NH), 12.02 (s, 1H, pyrrole$\mathrm{NH}), 12.17$ (s, 1H, N $\underline{\mathrm{HCO}}) \mathrm{ppm} ; \mathrm{HRMS} \mathrm{ESI}^{+} \mathrm{m} / \mathrm{z}$ for $\mathrm{C}_{30} \mathrm{H}_{31} \mathrm{~N}_{6} \mathrm{O}_{6} \mathrm{SCl}_{2}\left([\mathrm{M}+\mathrm{H}]^{+}\right)$: calcd 673.13974, found 673.13977; HPLC: $\mathrm{t}_{\mathrm{r}} 12.92 \mathrm{~min}(95 \%$ at $254 \mathrm{~nm})$.

6.2.2.3.9 N-((S)-1-(((S)-6-(3,4-Dichloro-5-methyl-1H-pyrrole-2-carboxamido)-4,5,6,7tetrahydrobenzo[d]thiazol-2-yl)amino)-3-methyl-1-oxobutan-2-yl)-5-((4-methoxybenzyl)oxy)4-oxo-1,4-dihydropyridine-2-carboxamide (42). Prepared from 32 (0.095 g, 0.198 mmol) and 10 (0.054 g, $0.198 \mathrm{mmol})$ according to the general procedure B. Product was purified by flash column chromatography using dichloromethane/methanol (20:1) as eluent. Yield $0.038 \mathrm{~g}$ (27 \%); brown solid; m. p. $153-155{ }^{\circ} \mathrm{C} ;{ }^{1} \mathrm{H}$ NMR (400 MHz, DMSO- $\left.d_{6}\right) \delta 0.91(\mathrm{t}, 6 \mathrm{H}, J=6.7 \mathrm{~Hz}$, $\left.2 \times \mathrm{CHC}_{3}\right), 1.92-2.07$ (m, 2H, H-7), 2.21-2.24 (m, 4H, $\underline{\mathrm{HCH}}_{3}$, pyrrole- $\left.\mathrm{CH}_{3}\right), 2.68-2.77$ (m,

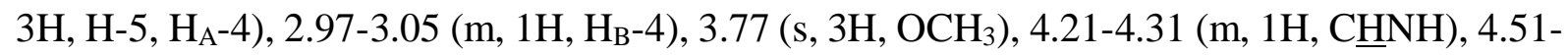
$4.60(\mathrm{~m}, 1 \mathrm{H}, \mathrm{COC} \underline{\mathrm{HNH}}), 5.19\left(\mathrm{~s}, 2 \mathrm{H}, \mathrm{OCH}_{2}\right), 6.93-6.99(\mathrm{~m}, 2 \mathrm{H}, \mathrm{Ar}-\mathrm{H}), 7.35$ (d, 1H, J = 7.9 Hz, CHN $\underline{H}), 7.39-7.45$ (m, 2H, Ar-H), 7.49 (s, 1H, pyridine-H), 8.26 (s, 1H, pyridine-H), 8.50- 
$8.57(\mathrm{~m}, 1 \mathrm{H}, \mathrm{COCHN} \underline{\mathrm{H}}), 10.90(\mathrm{~s}, 1 \mathrm{H}$, pyridine-NH) $12.01(\mathrm{~s}, 1 \mathrm{H}$, pyrrole-NH), $12.28(\mathrm{~s}, 1 \mathrm{H}$, N $\underline{H C O})$ ppm; HRMS ESI ${ }^{+} \mathrm{m} / \mathrm{z}$ for $\mathrm{C}_{32} \mathrm{H}_{35} \mathrm{~N}_{6} \mathrm{O}_{6} \mathrm{SCl}_{2}\left([\mathrm{M}+\mathrm{H}]^{+}\right)$: calcd 701.17104, found 701.17084; HPLC: $\mathrm{t}_{\mathrm{r}} 14.25 \mathrm{~min}(96 \%$ at $254 \mathrm{~nm})$.

$6.2 \cdot 2.3 .10$ (S)-N-(3-((6-(3,4-Dichloro-5-methyl-1H-pyrrole-2-carboxamido)-4,5,6,7tetrahydrobenzo[d]thiazol-2-yl)amino)-3-oxopropyl)-5-((4-methoxybenzyl)oxy)-4-oxo-1,4dihydropyridine-2-carboxamide (43). Prepared from 33 (0.178 g, $0.393 \mathrm{mmol})$ and $\mathbf{1 0}(0.180$ $\mathrm{g}, 0.393 \mathrm{mmol}$ ) according to the general procedure B. Crude product was recrystallized from ethyl acetate. Yield $0.066 \mathrm{~g}$ (25\%); brown solid; m. p. $184-186{ }^{\circ} \mathrm{C} ;[\alpha]_{\mathrm{D}}-8.8(c 0.24$, DMF); ${ }^{1} \mathrm{H}$ NMR (400 MHz, DMSO- $\left.d_{6}\right) \delta$ 1.89-2.07 (m, 2H, H-7), 2.19 (s, 3H, pyrrole- $\mathrm{CH}_{3}$ ), 2.65$2.77\left(\mathrm{~m}, 5 \mathrm{H}, \mathrm{H}-5, \mathrm{H}_{\mathrm{A}}-4, \mathrm{COC} \underline{\mathrm{H}}_{2}\right), 3.02\left(\mathrm{dd}, 1 \mathrm{H}, J_{1}=16.2 \mathrm{~Hz}, J_{2}=4.9 \mathrm{~Hz}, \mathrm{H}_{\mathrm{B}}-4\right), 3.55(\mathrm{dd}, 2 \mathrm{H}$, $\left.J_{l}=13.4 \mathrm{~Hz}, J_{2}=7.1 \mathrm{~Hz}, \underline{\mathrm{C}}_{2} \mathrm{NH}\right), 3.76\left(\mathrm{~s}, 3 \mathrm{H}, \mathrm{OCH}_{3}\right), 4.20-4.31(\mathrm{~m}, 1 \mathrm{H}, \mathrm{C} \underline{\mathrm{HNH}}), 5.17(\mathrm{~s}$, 2H, $\left.\mathrm{OCH}_{2}\right), 6.92-6.98(\mathrm{~m}, 2 \mathrm{H}, \mathrm{Ar}-\mathrm{H}), 7.34(\mathrm{~d}, 1 \mathrm{H}, J=7.7 \mathrm{~Hz}, \mathrm{CHN} \underline{\mathrm{H}}), 7.37-7.43(\mathrm{~m}, 2 \mathrm{H}, \mathrm{Ar}-$ $\mathrm{H}), 7.49\left(\mathrm{~s}, 1 \mathrm{H}\right.$, pyridine-H), $8.18\left(\mathrm{~s}, 1 \mathrm{H}\right.$, pyridine-H), $8.60\left(\mathrm{t}, 1 \mathrm{H}, J=6.1 \mathrm{~Hz}, \mathrm{CH}_{2} \mathrm{~N} \underline{\mathrm{H}}\right), 10.81$

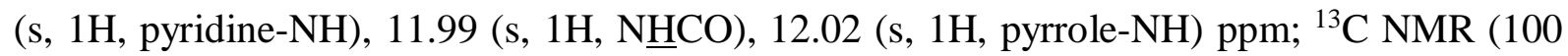
MHz, DMSO- $\left.d_{6}\right) \delta 11.1,24.7,28.5,28.8,35.3,35.4,45.7,55.6,70.8,108.5,110.1,110.4$, $114.3,119.5,119.7,127.8,128.8,130.3,135.5,143.7,144.9,146.1,154.8,155.8,158.7,159.6$ 164.2, 170.0 ppm; HRMS ESI ${ }^{-} \mathrm{m} / \mathrm{z}$ for $\mathrm{C}_{30} \mathrm{H}_{29} \mathrm{~N}_{6} \mathrm{O}_{6} \mathrm{SCl}_{2}\left([\mathrm{M}-\mathrm{H}]^{-}\right)$: calcd 671.12409, found 671.12640; HPLC: $\mathrm{t}_{\mathrm{r}} 12.22 \min (96 \%$ at $254 \mathrm{~nm})$.

6.2.2.4 General procedure D. Starting compound (1 mmol) was dissolved in acetic acid (50 $\mathrm{mL})$ and a solution of $1 \mathrm{M} \mathrm{HCl}$ in acetic acid $(20 \mathrm{~mL}, 20 \mathrm{mmol})$ was added dropwise. The mixture was stirred overnight at room temperature. Volume of the solvent was reduced in vacuo to only a few milliliters and diethyl ether $(100 \mathrm{~mL})$ was added. A precipitate was formed, which was filtered off and dried in vacuo. 
tetrahydrobenzo[d]thiazol-2-yl)amino)-2-oxoethyl)-5-hydroxy-4-oxo-1,4-dihydropyridine-2-

carboxamide (44). Prepared from $40(0.030 \mathrm{~g}, 0.0455 \mathrm{mmol})$ according to the general procedure D. Yield 0.019 g $(75 \%)$; pink solid; m. p. > $300{ }^{\circ} \mathrm{C} ;[\alpha]_{\mathrm{D}}-25.3(c$ 0.08, DMF $) ;{ }^{1} \mathrm{H}$ NMR $(400$ MHz, DMSO- $\left.d_{6}\right) \delta 1.93-2.07$ (m, 2H, H-7), $2.18\left(\mathrm{~s}, 3 \mathrm{H}\right.$, pyrrole- $\left.\mathrm{CH}_{3}\right), 2.66-2.76(\mathrm{~m}, 3 \mathrm{H}, \mathrm{H}-5$, $\left.\mathrm{H}_{\mathrm{A}}-4\right), 3.01\left(\mathrm{dd}, 1 \mathrm{H}, J_{1}=15.8 \mathrm{~Hz}, J_{2}=5.2 \mathrm{~Hz}, \mathrm{H}_{\mathrm{B}}-4\right), 4.18\left(\mathrm{~d}, 2 \mathrm{H}, J=5.7 \mathrm{~Hz}, \mathrm{CH}_{2} \mathrm{NH}\right), 4.22-$

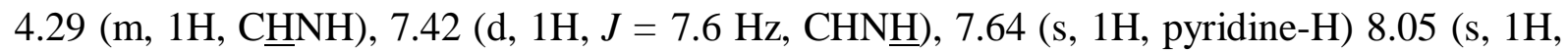

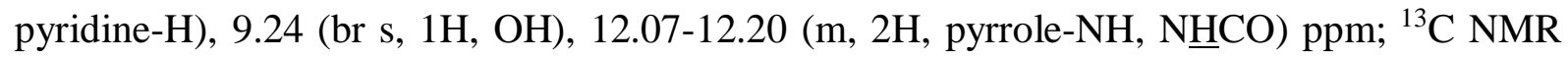
$\left(100 \mathrm{MHz}, \mathrm{DMSO}-d_{6}\right) \delta 11.1,24.7,28.6,28.8,42.9,45.6,108.5,110.7,111.1,119.6,119.8$, 127.7, 143.8, 146.6, 155.8, 158.7, 167.8 ppm; HRMS ESI- m/z for $\mathrm{C}_{21} \mathrm{H}_{19} \mathrm{~N}_{6} \mathrm{O}_{5} \mathrm{SCl}_{2}\left([\mathrm{M}-\mathrm{H}]^{-}\right)$: calcd 537.0515, found 537.0518; HPLC: $\mathrm{t}_{\mathrm{r}} 10.08 \mathrm{~min}(95 \%$ at $254 \mathrm{~nm})$.

6.2.2.4.2 N-((S)-1-(((S)-6-(3,4-Dichloro-5-methyl-1H-pyrrole-2-carboxamido)-4,5,6,7tetrahydrobenzo[d]thiazol-2-yl)amino)-1-oxopropan-2-yl)-5-hydroxy-4-oxo-1,4-

dihydropyridine-2-carboxamide (45). Prepared from 41 (0.027 g, 0.040 mmol) according to the general procedure D. Yield 0.014 g (62\%); orange solid; m. p. $207-209{ }^{\circ} \mathrm{C} ;[\alpha]_{\mathrm{D}}-51.8(c 0.07$, DMF); ${ }^{1} \mathrm{H}$ NMR (400 MHz, DMSO- $\left.d_{6}\right) \delta 1.44$ (d, 3H, $J=7.1 \mathrm{~Hz}, \mathrm{CHC}_{3}$ ), $1.89-2.07$ (m, 2H, H-7), 2.19 (s, 3H, pyrrole- $\mathrm{CH}_{3}$ ), 2.66-2.79 (m, 3H, H-5, $\left.\mathrm{H}_{\mathrm{A}}-4\right), 2.97-3.06$ (m, 1H, $\left.\mathrm{H}_{\mathrm{B}}-4\right), 4.20-$ 4.31 (m, 1H, $\left.\mathrm{CH}_{2} \mathrm{C} \underline{\mathrm{HNH}}\right), 4.62-4.72(\mathrm{~m}, 1 \mathrm{H}, \mathrm{COC} \underline{\mathrm{HNH}}), 7.38$ (d, 1H, J = 7.7 Hz, $\left.\mathrm{CH}_{2} \mathrm{CHN} \underline{\mathrm{H}}\right)$, $7.60(\mathrm{~s}, 1 \mathrm{H}$, pyridine-H), 8.02 (s, 1H, pyridine-H), $8.93(\mathrm{~s}, 1 \mathrm{H}, \mathrm{OH}), 12.06(\mathrm{~s}, 1 \mathrm{H}$, pyrrole-NH), 12.18 (br s, $1 \mathrm{H}, \mathrm{N} \underline{\mathrm{HCO}}$ ) ppm; ${ }^{13} \mathrm{C}$ NMR (100 MHz, DMSO-d $) \delta 11.1,18.1,24.7,28.6,28.8$, 45.6, 49.7, 108.5, 110.6, 119.6, 120.0, 127.7, 143.9, 146.7, 158.7, 171.1 ppm; HRMS ESI ${ }^{+}$m/z for $\mathrm{C}_{22} \mathrm{H}_{23} \mathrm{~N}_{6} \mathrm{O}_{5} \mathrm{SCl}_{2}\left([\mathrm{M}+\mathrm{H}]^{+}\right)$: calcd 553.08222, found 553.08264; HPLC: $\mathrm{t}_{\mathrm{r}} 10.27$ min (96 \% at $254 \mathrm{~nm})$.

6.2.2.4.3 N-((S)-1-(((S)-6-(3,4-Dichloro-5-methyl-1H-pyrrole-2-carboxamido)-4,5,6,7tetrahydrobenzo[d]thiazol-2-yl)amino)-3-methyl-1-oxobutan-2-yl)-5-hydroxy-4-oxo-1,4- 
dihydropyridine-2-carboxamide (46). Prepared from 42 (0.033 g, $0.047 \mathrm{mmol})$ according to the general procedure D. Yield $0.021 \mathrm{~g}(77 \%)$; brown solid; m. p. $213-215^{\circ} \mathrm{C} ;[\alpha]_{\mathrm{D}}+8.9(c 0.13$, DMF); ${ }^{1} \mathrm{H}$ NMR (400 MHz, DMSO- $\left.d_{6}\right) \delta 0.92\left(\mathrm{~d}, 4 \mathrm{H}, J=6.6 \mathrm{~Hz}, \mathrm{CHCH}_{3}\right), 1.10(\mathrm{t}, 2 \mathrm{H}, J=$ 7.0 Hz, $\mathrm{CHCH}_{3}$ ), 1.89-2.07 (m, 2H, H-7), 2.13-2.24 (m, 4H, $\underline{\mathrm{HCH}}_{3}$, pyrrole- $\mathrm{CH}_{3}$ ), 2.66-2.79 (m, 3H, H-5, $\left.\mathrm{H}_{\mathrm{A}}-4\right), 2.97-3.06\left(\mathrm{~m}, 1 \mathrm{H}, \mathrm{H}_{\mathrm{B}}-4\right), 4.20-4.31$ (m, 1H, $\left.\mathrm{CH}_{2} \mathrm{CHNH}\right), 4.51-4.59$ (m, $1 \mathrm{H}, \mathrm{COC} \underline{\mathrm{HNH}}), 7.39$ (d, 1H, $\left.J=7.6 \mathrm{~Hz}, \mathrm{CH}_{2} \mathrm{CHN} \underline{\mathrm{H}}\right), 7.58(\mathrm{~s}, 1 \mathrm{H}$, pyridine-H), $8.04(\mathrm{~s}, 1 \mathrm{H}$,

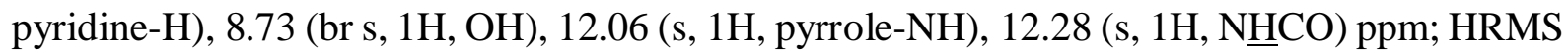
$\mathrm{ESI}^{+} \mathrm{m} / \mathrm{z}$ for $\mathrm{C}_{24} \mathrm{H}_{27} \mathrm{~N}_{6} \mathrm{O}_{5} \mathrm{SCl}_{2}\left([\mathrm{M}+\mathrm{H}]^{+}\right)$: calcd 581.11352, found 581.11365; HPLC: $\mathrm{t}_{\mathrm{r}} 11.09$ $\min (95 \%$ at $254 \mathrm{~nm})$.

6.2.2.4.4

(S)-N-(3-((6-(3,4-Dichloro-5-methyl-1H-pyrrole-2-carboxamido)-4,5,6,7tetrahydrobenzo[d]thiazol-2-yl)amino)-3-oxopropyl)-5-hydroxy-4-oxo-1,4-dihydropyridine-2carboxamide (47). Prepared from $43(0.054 \mathrm{~g}, 0.080 \mathrm{mmol})$ according to the general procedure D. Yield $0.036 \mathrm{~g}(80 \%)$; brown solid; m. p. $220-222{ }^{\circ} \mathrm{C} ;[\alpha]_{\mathrm{D}}-4.7\left(c 0.24\right.$, DMF); ${ }^{1} \mathrm{H}$ NMR $\left(400 \mathrm{MHz}, \mathrm{DMSO}-d_{6}\right) \delta 1.87-1.97\left(\mathrm{~m}, 1 \mathrm{H}, \mathrm{H}_{\mathrm{A}}-7\right), 1.97-2.07\left(\mathrm{~m}, 1 \mathrm{H}, \mathrm{H}_{\mathrm{B}}-7\right), 2.19$ (s, 3H, pyrrole$\left.\mathrm{CH}_{3}\right), 2.65-2.80\left(\mathrm{~m}, 5 \mathrm{H}, \mathrm{H}-5, \mathrm{H}_{\mathrm{A}}-4, \mathrm{COCH}_{2}\right), 2.96-3.05\left(\mathrm{~m}, 1 \mathrm{H}, \mathrm{H}_{\mathrm{B}}-4\right), 4.20-4.30(\mathrm{~m}, 1 \mathrm{H}$,

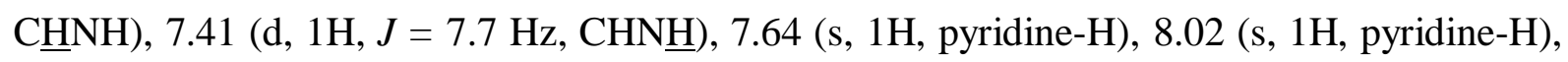
9.06 (br s, 1H, OH), 12.03 (s, 1H, N $\underline{H C O}), 12.10$ (s, 1H, pyrrole-NH) ppm; ${ }^{13} \mathrm{C}$ NMR (100 MHz, DMSO- $\left.d_{6}\right) \delta 11.1,24.8,28.6,28.8,35.0,36.1,45.7,108.5,110.8,111.3,119.6,127.7$, 143.7, 146.9, 155.9, 158.7, 169.5 ppm; HRMS ESI ${ }^{+} \mathrm{m} / \mathrm{z}$ for $\mathrm{C}_{22} \mathrm{H}_{23} \mathrm{~N}_{6} \mathrm{O}_{5} \mathrm{SCl}_{2}\left([\mathrm{M}+\mathrm{H}]^{+}\right)$: calcd 553.08222, found 553.08258; HPLC: $\mathrm{t}_{\mathrm{r}} 10.04 \mathrm{~min}(95 \%$ at $254 \mathrm{~nm})$.

\subsection{Determination of inhibitory activities against $E$. coli DNA gyrase}

The assays for determination of $\mathrm{IC}_{50}$ against $E$. coli DNA gyrase were performed according to previously reported procedures [10]. IC $_{50}$ values were determined with seven concentrations of the inhibitors. GraphPad Prism program was used for calculating an $\mathrm{IC}_{50}$ value, which 
represents the concentration of inhibitor where the activity of the enzyme is reduced by $50 \%$. $\mathrm{IC}_{50}$ values were determined in three independent measurements, and their average value is given as a final result.

\subsection{Determination of antibacterial activities}

The clinical microbiology control strains of E. faecalis (ATCC 29212), S. aureus (ATCC 25923 and 29213), E. coli (ATCC 25922), P. aeruginosa (ATCC 27853) and A. baumannii (ATCC 19606) were obtained from Microbiologics Inc. (St. Cloud, MN, USA). The single-gene knockout mutant strains of E. coli JW5503 (tolC knock-out) and JD17464 (lpxC knock-out) were obtained from the E. coli collection of the National BioResource Project at the National Institute of Genetics (Japan) [35]. To determine the antibacterial activities, broth microdilution assays were carried out in 96-well plates following the Clinical and Laboratory Standards Institute guidelines [27]. Preliminary screening was performed at $50 \mu \mathrm{M}$, and growth inhibition was measured after $24 \mathrm{~h}$ incubation. For compounds displaying $>90 \%$ growth inhibition in the preliminary assay, the MICs were determined in dose-response assays (from at least two independent experiments, each with three replicates per concentration, except when stated otherwise). Cation-adjusted Mueller-Hinton broth (CAMHB, BD) was used in all assays. CAMHB was prepared according to manufacturer's instructions and iron-depleted CAMHB (ID-CAMHB) according to CLSI guidelines [36]. Iron-depleted conditions mimic the conditions faced by bacteria during infections of human tissues and fluids. Thus, the use of iron-depleted conditions was required to induce the ferric iron transport. In brief, ID-CAMHB was prepared by adding $100 \mathrm{~g}$ of Chelex 100 resin (Bio-Rad) to $1 \mathrm{~L}$ of autoclaved CAMHB and stirred for $2 \mathrm{~h}$ at room temperature, in order to remove cations from the medium. Then, broth was filtered using a $0.2 \mu \mathrm{m}$ filter to remove the resin and $\mathrm{pH}$ adjusted to 7.3 using $5 \mathrm{M}$ hydrochloric acid. ID-CAMHB was then supplemented with calcium $\left(\mathrm{CaCl}_{2}\right)$, magnesium 
$\left(\mathrm{MgCl}_{2}\right)$ and zinc $\left(\mathrm{ZnSO}_{4}\right)$ to final concentrations of $22.5 \mu \mathrm{g} / \mathrm{mL}, 11.25 \mu \mathrm{g} / \mathrm{mL}$ and $0.56 \mu \mathrm{g} / \mathrm{mL}$, respectively, and filtered again. To evaluate the effect of iron concentration on antibacterial effect of compounds, an appropriate amount of iron (III) chloride was added to ID-CAMHB, and this supplemented media was used as control CAMHB. The iron concentration in CAMHB and ID-CAMHB were approximately $0.2 \mathrm{mg} / \mathrm{L}$ and $\leq 0.03 \mathrm{mg} / \mathrm{L}$, respectively. Cefiderocol MICs were determined against E. coli, $P$. aeruginosa and A. baumannii, and used as quality control in the assays. Cefiderocol was purchased from MedChemExpress Europe.

Molecular docking. Three-dimensional models of the designed DNA gyrase inhibitors and their conjugates with siderophore mimics were built in Chem3D 18.0 (PerkinElmer Inc., Massachusetts, USA). The geometries and charges of the ligands were optimized using the MM2 force field and partial atomic charges were assigned. The energy was minimized until the gradient value was smaller than $0.001 \mathrm{kcal} /($ mol $\times \AA$ ). Molecular docking calculations were performed in Schrödinger Release 2019-1 (Schrödinger, LLC, New York, NY, USA, 2019). Crystal structure of E. coli DNA gyrase B in complex with bithiazole inhibitor (PDB entry: 4DUH) was retrieved from Protein Data Bank. Protein was then prepared by Protein Preparation Wizard using default settings. Receptor grid was calculated for the ligand-binding site and designed compounds were docked using Glide XP protocol as implemented in Schrödinger Release 2019-1 (Glide, Schrödinger, LLC, New York, NY, USA, 2019). The highest ranked docking pose was used for visualization.

\section{Associated content}

\section{Appendix A. Supplementary Information}

The full data for the synthetic procedures of the siderophore mimics, and ${ }^{1} \mathrm{H}$ and ${ }^{13} \mathrm{C}$ NMR spectra are given in the Supplementary Information. This material is available via the internet. 


\section{Author information}

\section{Corresponding author: Tihomir Tomašič}

E-mail: thomir.tomasic@ffa.uni-lj.si

Tel: $+386-1-4769556$

Fax: +386-1-4258031

\section{Author contributions}

The manuscript was written with contributions from all of the authors. All of the authors have given approval to the final version of the manuscript.

\section{Funding sources}

The work was funded by the Slovenian Research Agency (Grant No. P1-0208) and the Academy of Finland (Grant Nos. 277001, 304697, 312503).

\section{Conflicts of interest}

The authors declare that they have no conflicts of interest, including no financial, personal or other relationships with other people or organizations.

\section{Acknowledgements}

The authors thank Dušan Žigon (Mass Spectrometry Centre, Jožef Stefan Institute, Ljubljana, Slovenia) for the mass spectra, Heidi Mäkkylä, Cristina Carbonell Duacastella and Heli Parviainen for their technical assistance in the antibacterial assays, and Christopher Berrie for scientific editing of the manuscript. 


\section{References}

[1] G.V. Asokan, R.K. Kasimanickam, Emerging infectious diseases, antimicrobial resistance and millennium development goals: resolving the challenges through one health, Cent. Asian J. Glob. Health 2 (2013) 76.

[2] E. Toner, A. Adalja, G.K. Gronvall, A. Cicero, T.V. Inglesby, Antimicrobial resistance is a global health emergency, Health Secur. 13 (2015) 153-155.

[3] World Health Organization, WHO publishes list of bacteria for which new antibiotics are urgently needed, https://www.who.int/news-room/detail/27-02-2017-who-publishes-list-ofbacteria-for-which-new-antibiotics-are-urgently-needed, 2017 (accessed 27 August 2019).

[4] P. Fernandes, E. Martens, Antibiotics in late clinical development, Biochem. Pharmacol. 133 (2017) 152-163.

[5] M. Mazer-Amishahi, A. Pourmand, L. May, Newly approved antibiotics and antibiotics reserved for resistant infections: implications for emergency medicine, Am. J. Emerg. Med. 35 (2017) 154-158.

[6] X.Z. Li, P. Plesiat, H. Nikaido, The challenge of efflux-mediated antibiotic resistance in Gram-negative bacteria, Clin. Microbiol. Rev. 28 (2015) 337-418.

[7] T. Tomašič, L. Peterlin Mašič, Prospects for developing new antibacterials targeting bacterial type IIA topoisomerases, Curr. Top. Med. Chem. 14 (2014) 130-151.

[8] G.S. Bisacchi, J.I. Manchester, A new-class antibacterial-almost. Lessons in drug discovery and development: a critical analysis of more than 50 years of effort toward ATPase inhibitors of DNA gyrase and topoisomerase IV, ACS Infect. Dis. 1 (2015) 4-41.

[9] M. Durcik, T. Tomašič, N. Zidar, A. Zega, D. Kikelj, L. Peterlin Mašič, J. Ilaš, ATPcompetitive DNA gyrase and topoisomerase IV inhibitors as antibacterial agents, Expert Opin. Ther. Pat. 29 (2019) 171-180. 
[10] T. Tomašič, S. Katsamakas, Ž. Hodnik, J. Ilaš, M. Brvar, T. Šolmajer, S. Montalvao, P. Tammela, M. Banjanac, G. Ergović, M. Anderluh, L. Peterlin Mašič, D. Kikelj, Discovery of 4,5,6,7-tetrahydrobenzo[1,2- $d]$ thiazoles as novel DNA gyrase inhibitors targeting the ATPbinding site, J. Med. Chem. 58 (2015) 5501-5521.

[11] T. Tomašič, M. Mirt, M. Barančokova, J. Ilaš, N. Zidar, P. Tammela, D. Kikelj, Design, synthesis and biological evaluation of 4,5-dibromo- $N$-(thiazol-2-yl)-1H-pyrrole-2-carboxamide derivatives as novel DNA gyrase inhibitors, Bioorg. Med. Chem. 25 (2017) 338-349.

[12] M. Gjorgjieva, T. Tomašič, M. Barančokova, S. Katsamakas, J. Ilaš, P. Tammela, L. Peterlin Mašič, D. Kikelj, Discovery of benzothiazole scaffold-based DNA gyrase B inhibitors, J. Med. Chem. 59 (2016) 8941-8954.

[13] D. Benedetto Tiz, Ž. Skok, M. Durcik, T. Tomašič, L. Peterlin Mašič, J. Ilaš, A. Zega, G. Draskovits, T. Revesz, A. Nyerges, C. Pal, C.D. Cruz, P. Tammela, D. Žigon, D. Kikelj, N. Zidar, An optimised series of substituted $N$-phenylpyrrolamides as DNA gyrase B inhibitors, Eur. J. Med. Chem. 167 (2019) 269-290.

[14] I.A. Yule, L.G. Czaplewski, S. Pommier, D.T. Davies, S.K. Narramore, C.W. Fishwick, Pyridine-3-carboxamide-6-yl-ureas as novel inhibitors of bacterial DNA gyrase: structure based design, synthesis, SAR and antimicrobial activity, Eur. J. Med. Chem. 86 (2014) 31-38.

[15] T. Tomašič, M. Barančokova, N. Zidar, J. Ilaš, P. Tammela, D. Kikelj, Design, synthesis, and biological evaluation of 1-ethyl-3-(thiazol-2-yl)urea derivatives as Escherichia coli DNA gyrase inhibitors, Arch. Pharm. (Weinheim) 351 (2018).

[16] D. Benedetto Tiz, D. Kikelj, N. Zidar, Overcoming problems of poor drug penetration into bacteria, Expert Opin. Drug Discov. 13 (2018) 497-507.

[17] H.I. Zgurskaya, C.A. Lopez, S. Gnanakaran, Permeability barrier of Gram-negative cell envelopes and approaches to bypass it, ACS Infect. Dis. 1 (2015) 512-522. 
[18] E. Ahmed, S.J. Holmstrom, Siderophores in environmental research: roles and applications, Microb. Biotechnol. 7 (2014) 196-208.

[19] R. Golonka, B.S. Yeoh, M. Vijay-Kumar, The iron tug-of-war between bacterial siderophores and innate immunity, J. Innate Immun. 11 (2019) 249-262.

[20] U. Bilitewski, J.A.V. Blodgett, A.K. Duhme-Klair, S. Dallavalle, S. Laschat, A. Routledge, R. Schobert, Chemical and biological aspects of nutritional immunity-perspectives for new antiinfectives that target iron uptake systems, Angew. Chem. Int. Ed. Engl. 56 (2017) 14360-14382. [21] G.L. Mislin, I.J. Schalk, Siderophore-dependent iron uptake systems as gates for antibiotic Trojan horse strategies against Pseudomonas aeruginosa, Metallomics 6 (2014) 408-420.

[22] M.E. Falagas, A.D. Mavroudis, K.Z. Vardakas, The antibiotic pipeline for multi-drug resistant Gram negative bacteria: what can we expect?, Expert Rev. Anti-infect. Ther. 14 (2016) 747-763.

[23] G.G. Zhanel, A.R. Golden, S. Zelenitsky, K. Wiebe, C.K. Lawrence, H.J. Adam, T. Idowu, R. Domalaon, F. Schweizer, M.A. Zhanel, P.R.S. Lagace-Wiens, A.J. Walkty, A. Noreddin, J.P. Lynch Iii, J.A. Karlowsky, Cefiderocol: a siderophore cephalosporin with activity against carbapenem-resistant and multidrug-resistant Gram-negative Bacilli, Drugs 79 (2019) 271-289. [24] U.S. Food \& Drug administration, FDA approves new antibacterial drug to treat complicated urinary tract infections as part of ongoing efforts to address antimicrobial resistance, $\quad$ https://www.fda.gov/news-events/press-announcements/fda-approves-newantibacterial-drug-treat-complicated-urinary-tract-infections-part-ongoing-efforts, $\quad 2019$ (accessed 18 December 2019).

[25] N. Zidar, H. Macut, T. Tomašič, M. Brvar, S. Montalvao, P. Tammela, T. Šolmajer, L. Peterlin Mašič, J. Ilaš, D. Kikelj, $N$-Phenyl-4,5-dibromopyrrolamides and $N$-phenylindolamides as ATP competitive DNA gyrase B inhibitors: design, synthesis, and evaluation, J. Med. Chem. 58 (2015) 6179-6194. 
[26] B.A. Sherer, K. Hull, O. Green, G. Basarab, S. Hauck, P. Hill, J.T. Loch 3rd, G. Mullen, S. Bist, J. Bryant, A. Boriack-Sjodin, J. Read, N. DeGrace, M. Uria-Nickelsen, R.N. Illingworth, A.E. Eakin, Pyrrolamide DNA gyrase inhibitors: optimization of antibacterial activity and efficacy, Bioorg. Med. Chem. Lett. 21 (2011) 7416-7420.

[27] Methods for dilution antimicrobial susceptibility tests for bacteria that grow aerobically, approved standard, CLSI, Wayne, Pennsylvania, 2012.

[28] M.G.P. Page, Siderophore conjugates, Ann. NY Acad. Sci. 1277 (2013) 115-126.

[29] M.D. Huband, A. Ito, M. Tsuji, H.S. Sader, K.A. Fedler, R.K. Flamm, Cefiderocol MIC quality control ranges in iron-depleted cation-adjusted Mueller-Hinton broth using a CLSI M23-A4 multi-laboratory study design, Diagn. Microbiol. Infect. Dis. 88 (2017) 198-200.

[30] M.A. Scorciapino, G. Malloci, I. Serra, S. Milenkovic, L. Moynie, J.H. Naismith, E. Desarbre, M.G.P. Page, M. Ceccarelli, Complexes formed by the siderophore-based monosulfactam antibiotic BAL30072 and their interaction with the outer membrane receptor PiuA of $P$. aeruginosa, Biometals 32 (2019) 155-170.

[31] L. Moynie, I. Serra, M.A. Scorciapino, E. Oueis, M.G. Page, M. Ceccarelli, J.H. Naismith, Preacinetobactin not acinetobactin is essential for iron uptake by the BauA transporter of the pathogen Acinetobacter baumannii, Elife 7 (2018).

[32] L. Moynie, S. Milenkovic, G.L.A. Mislin, V. Gasser, G. Malloci, E. Baco, R.P. McCaughan, M.G.P. Page, I.J. Schalk, M. Ceccarelli, J.H. Naismith, The complex of ferricenterobactin with its transporter from Pseudomonas aeruginosa suggests a two-site model, Nat. Commun. 10 (2019) 3673.

[33] Y.L. Cho, H.J. Heo, K.M. Oh, H.S. Lee, C.S. Park, S.E. Chae, J.Y. Yun, H.J. Kwon, Y.J. Yang, D.H. Kang, Y.Z. Kim, S.H. Woo, T.K. Park, US Patent, 2012, 0264727 A1.

[34] M.E. Flanagan, S.J. Brickner, M. Lall, J. Casavant, L. Deschenes, S.M. Finegan, D.M. George, K. Granskog, J.R. Hardink, M.D. Huband, T. Hoang, L. Lamb, A. Marra, M. Mitton- 
Fry, J.P. Mueller, L.M. Mullins, M.C. Noe, J.P. O'Donnell, D. Pattavina, J.B. Penzien, B.P. Schuff, J. Sun, D.A. Whipple, J. Young, T.D. Gootz, Preparation, Gram-negative antibacterial activity, and hydrolytic stability of novel siderophore-conjugated monocarbam diols, ACS Med. Chem. Lett. 2 (2011) 385-390.

[35] T. Baba, T. Ara, M. Hasegawa, Y. Takai, Y. Okumura, M. Baba, K.A. Datsenko, M. Tomita, B.L. Wanner, H. Mori, Construction of Escherichia coli K-12 in-frame, single-gene knockout mutants: the Keio collection, Mol. Syst. Biol. 2 (2006) 20060008.

[36] Subcommittee on antimicrobial susceptibility testing, meeting minutes and presentations, CLSI, 2016. 


\section{Supplementary Information for:}

Design, Synthesis and Biological Evaluation of Novel DNA Gyrase Inhibitors and their Siderophore Mimic Conjugates

Andraž Lamut, ${ }^{\mathrm{a}}$ Cristina D. Cruz, ${ }^{\mathrm{b}}$ Žiga Skok, ${ }^{\mathrm{a}}$ Michaela Barančoková, ${ }^{\mathrm{a}}$ Nace Zidar, ${ }^{\mathrm{a}}$ Anamarija Zega, ${ }^{a}$ Lucija Peterlin Mašič, ${ }^{a}$ Janez Ilaš, ${ }^{a}$ Päivi Tammela, ${ }^{b}$ Danijel Kikelj, ${ }^{a}$ and Tihomir Tomašič ${ }^{\mathrm{a}, *}$

${ }^{a}$ University of Ljubljana, Faculty of Pharmacy, Aškerčeva cesta 7, 1000 Ljubljana, Slovenia

${ }^{b}$ Drug Research Program, Division of Pharmaceutical Biosciences, Faculty of Pharmacy, University of Helsinki, P.O. Box 56 (Viikinkaari 5 E), FI-00014 Helsinki, Finland

*Corresponding author: Tihomir Tomašič

University of Ljubljana, Faculty of Pharmacy,

Aškerčeva cesta 7, 1000 Ljubljana, Slovenia

Tel: +386-1-4769556

E-mail: tihomir.tomasic@ffa.uni-lj.si 


\section{ENZYME INHIBITION}

Table S1. Structures of the $(S)$-4,5,6,7-tetrahydrobenzo[ $d]$ thiazol-6-yl-1 $H$-pyrrole-2-carboxamide derivatives and their inhibition of Escherichia coli DNA gyrase.

Compound


23

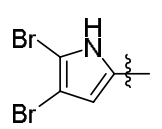

(s)<smiles>Brc1cc[nH]c1Br</smiles>

$\mathrm{NH}_{3}{ }^{+} \mathrm{Cr}$

$1.1 \pm 0.1$

30
$\mathrm{O}_{3 / 2} \mathrm{NH}^{+} \mathrm{Cl}^{-}$

$1.8 \pm 0.2$

${ }^{a}$ Novobiocin used as positive control in enzyme assays 
Table S2. Structures of the $(S)-4,5,6,7$-tetrahydrobenzo[ $d]$ thiazol-6-yl-1H-pyrrole-2-carboxamide GyrB inhibitor-siderophore mimic conjugates $(\mathbf{1 5 - 1 7}, \mathbf{3 4 - 3 9}, \mathbf{4 4 - 4 7 )}$ and their inhibition of Escherichia coli DNA gyrase.

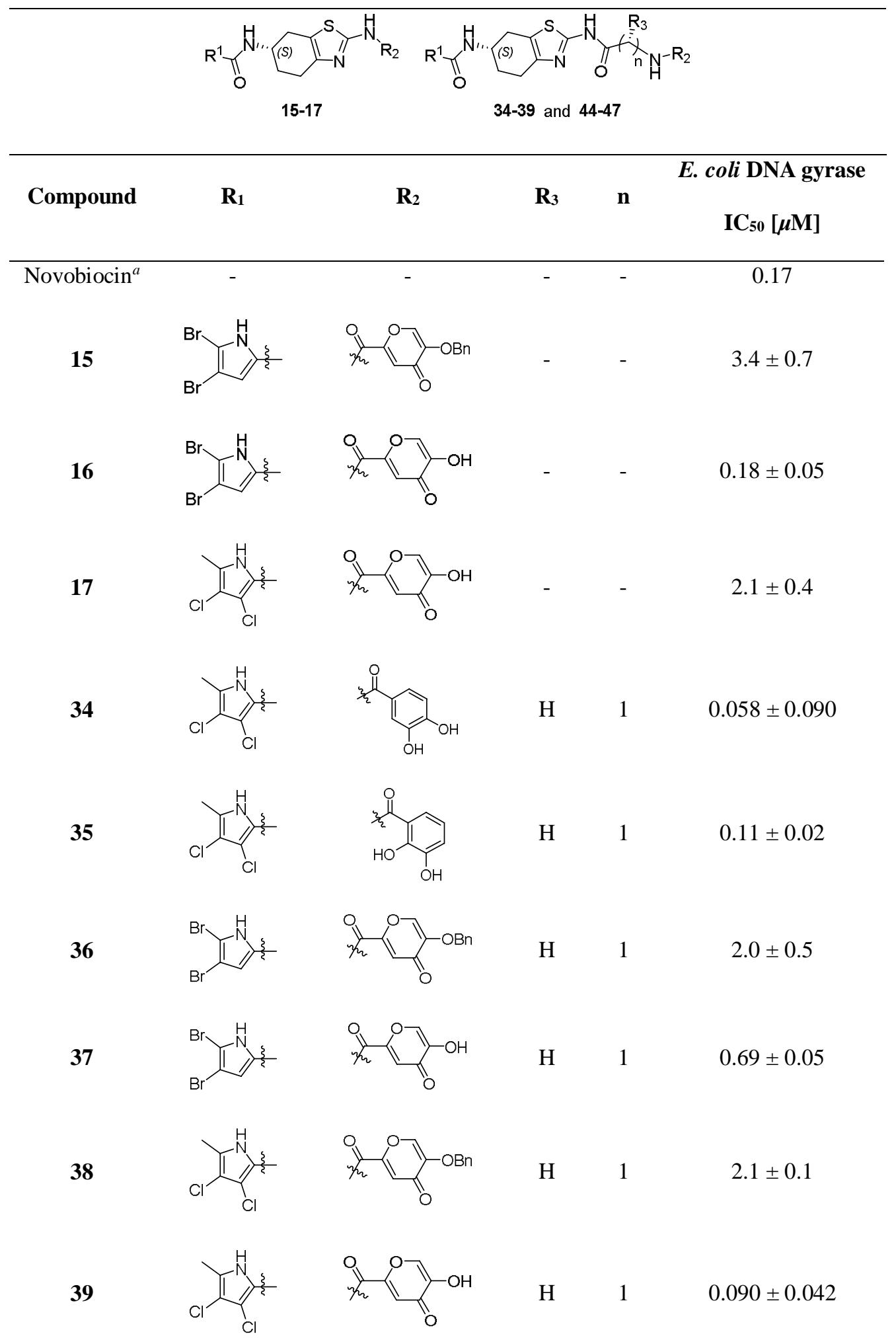


44<smiles>Cc1[nH]c(Cl)c(Cl)c1Cl</smiles>

45<smiles>Cc1[nH]cc(Cl)c1Cl</smiles>

46<smiles>Cc1[nH][Y]c(Cl)c1Cl</smiles>

47<smiles>Cc1[nH]cc(Cl)c1Cl</smiles><smiles>[Y]C(=O)C1C=CC(=O)C(O)=CN1</smiles>
$i$-Pr<smiles>CC(=O)C1C=CC(=O)C(O)=CN1</smiles>
$\mathrm{Me}$ 1 $0.26 \pm 0.05$<smiles>CC(=O)c1cc(=O)c(O)c[nH]1</smiles>

H 1 $1.6 \pm 0.1$<smiles>[X]C(=O)c1cc(=O)c(O)c[nH]1</smiles>

H 2 $0.35 \pm 0.09$

${ }^{a}$ Novobiocin used as positive control in enzyme assays 


\section{ANTIBACTERIAL ACTIVITY}

120

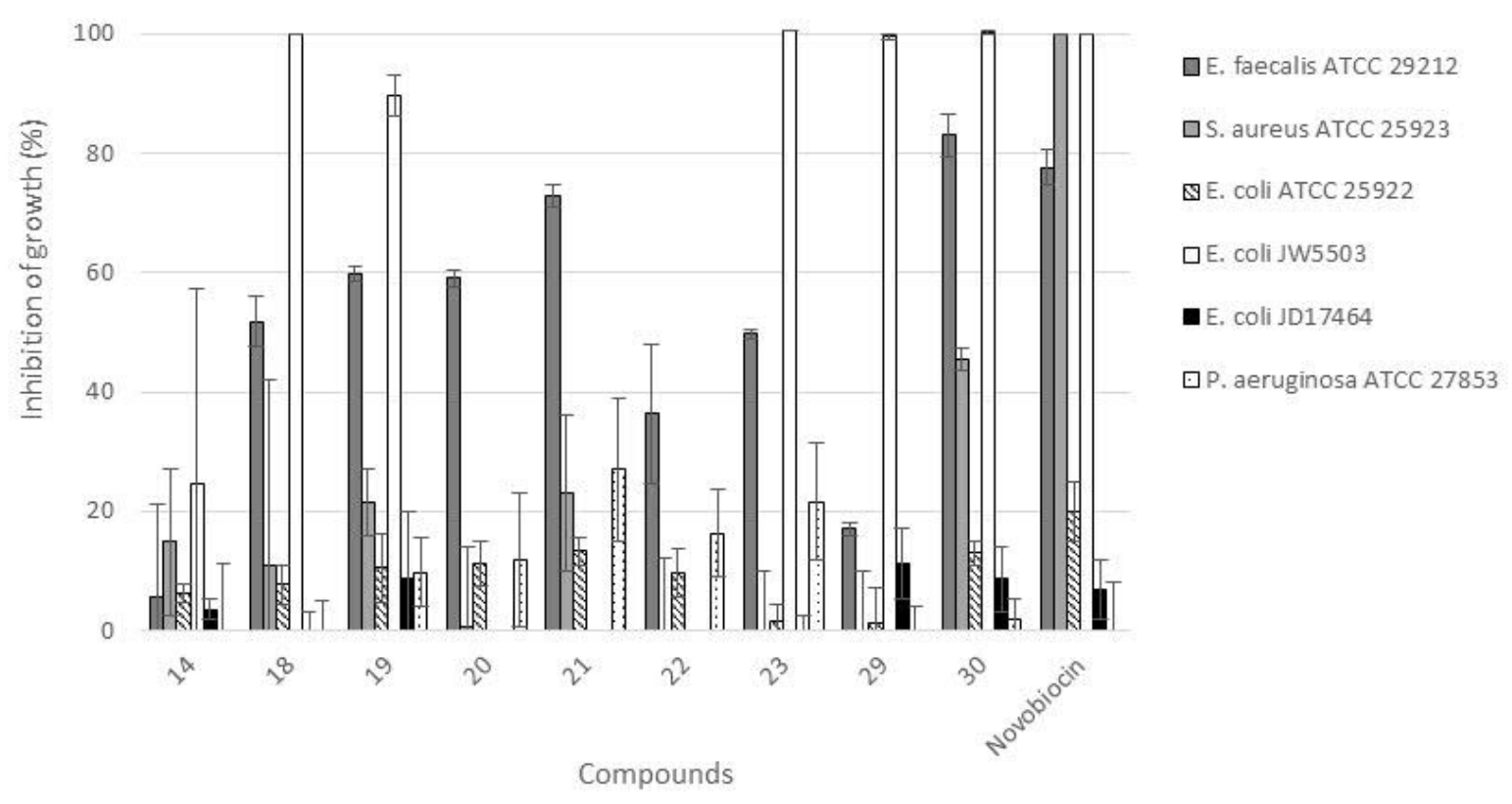

Figure S1. Antibacterial activities of new DNA gyrase inhibitors 14, 18-23, 29 and 30 against selected Grampositive and Gram-negative bacterial strains in CAMHB media. Inhibition of growth was determined at $50 \mu \mathrm{M}$ of the tested compound relative to untreated control. Novobiocin at $4 \mu \mathrm{g} / \mathrm{mL}$ was used as a positive control. Compounds 20-22 were not tested against E. coli mutant strains. 


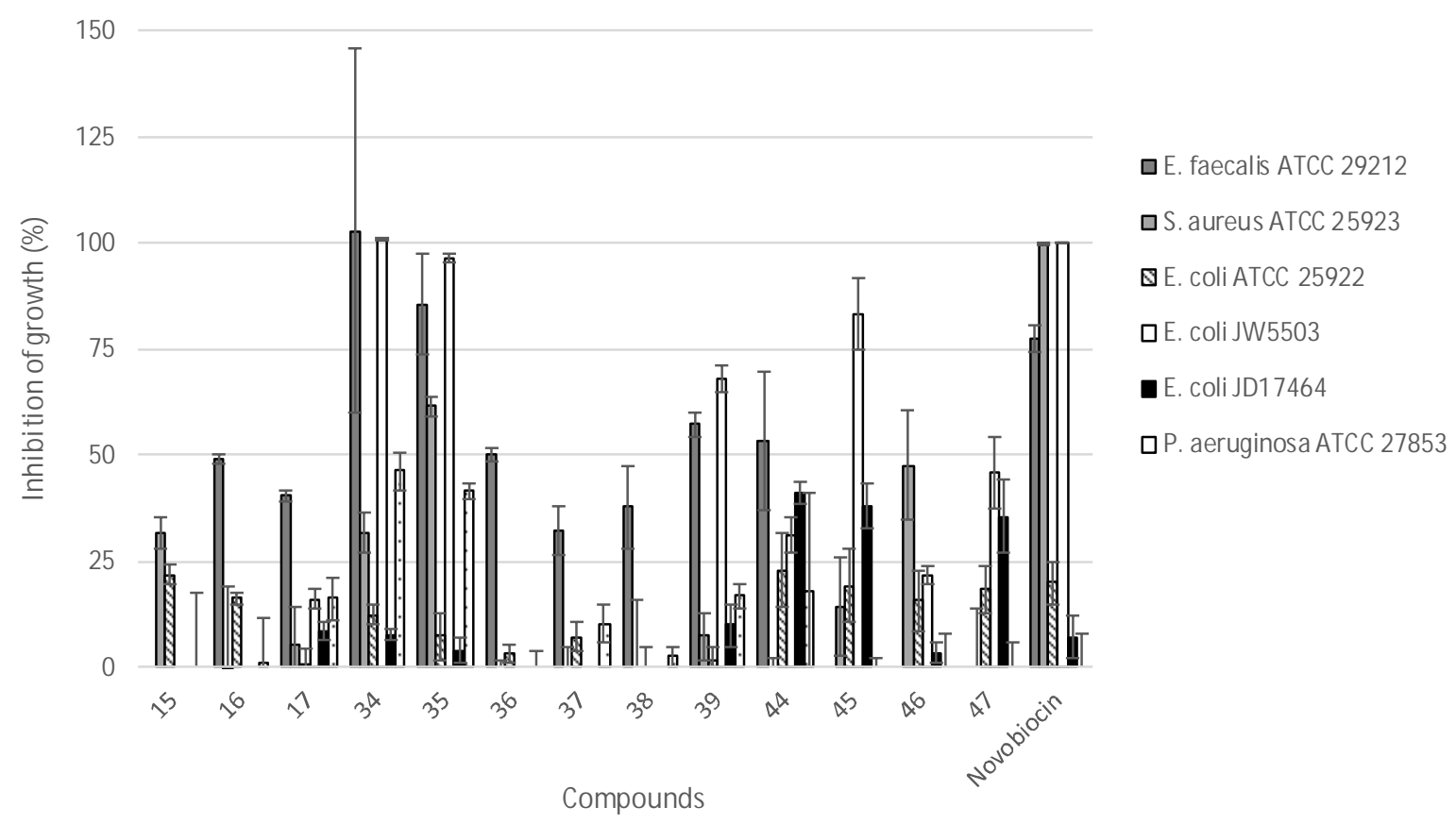

Figure S2. Antibacterial activities of new DNA gyrase inhibitor-siderophore mimic conjugates 15-17, 34-39 and 44-47 against selected Gram-positive and Gram-negative bacterial strains, in CAMHB media. Inhibition of growth was determined at $50 \mu \mathrm{M}$ of the tested compound relative to untreated control. Novobiocin at $4 \mu \mathrm{g} / \mathrm{mL}$ was used as a positive control. Compounds 15, 16 and $\mathbf{3 6 - 3 8}$ were not tested against E. coli mutant strains. Compounds $\mathbf{1 5}$, 16, 34, 35, 39 and 44-47 were tested against S. aureus ATCC29213. 
Table S3. Minimum inhibitory concentration (MIC) of the most potent compounds from initial screening at 50 $\mu \mathrm{M}$ against E. faecalis ATCC 29212 and E. coli JW5503.

\begin{tabular}{ccc}
\hline Compounds & \multicolumn{2}{c}{ MIC $[\mu \mathrm{g} / \mathrm{mL}]^{\mathrm{a}}$} \\
\hline & E. faecalis & E. coli \\
& ATCC 29212 & JW5503 \\
\hline Inhibitor + linker & & 13 \\
$\mathbf{1 8}$ & $\mathrm{nt}^{\mathrm{b}}$ & 1 \\
$\mathbf{1 9}$ & $\mathrm{nt}$ & 16 \\
$\mathbf{2 9}$ & $\mathrm{nt}$ & 26 \\
$\mathbf{3 0}$ & $\mathrm{nt}$ & 11
\end{tabular}

Inhibitor + siderophore mimic

$34>40 \quad 14$

$35 \quad 40 \quad 14$

\section{Controls}

Ciprofloxacin $\quad 1 \quad 0.005$

Novobiocin $\quad>4$

${ }^{\mathrm{a}} \mathrm{MIC}$, minimum inhibitory concentration that inhibits the growth of bacteria by $\geq 90 \%$

bnot tested: screening assays performed with compound's concentration at $50 \mu \mathrm{M}$ did not reach inhibition $\geq 90 \%$ 
Table S4. Antibacterial activities of the DNA gyrase inhibitors and inhibitor-siderophore mimic conjugates against Escherichia coli, Pseudomonas aeruginosa and Acinetobacter baumannii in cation-adjusted Mueller-Hinton broth as either iron-depleted $(-\mathrm{Fe})$ or iron-supplemented $(+\mathrm{Fe})$.

\begin{tabular}{|c|c|c|c|c|c|c|}
\hline & \multicolumn{2}{|c|}{ E. coli ATCC 25922} & \multicolumn{2}{|c|}{ P. aeruginosa ATCC 27853} & \multicolumn{2}{|c|}{ A. baumannii ATCC 19606} \\
\hline & $-\mathrm{Fe}$ & $+\mathrm{Fe}$ & $-\mathrm{Fe}$ & $+\mathrm{Fe}$ & $-\mathrm{Fe}$ & $+\mathrm{Fe}$ \\
\hline \multicolumn{7}{|c|}{ Minimum inhibitory concentrations $[\mu \mathrm{g} / \mathrm{mL}]^{\mathrm{a}}$} \\
\hline Cefiderocol $^{\mathbf{c}}$ & 0.25 & 0.25 & 0.5 & 1 & 0.25 & 2 \\
\hline Ceftazidime $^{\mathbf{d}}$ & 0.25 & 0.25 & 2 & 2 & $>4$ & $>4$ \\
\hline \multicolumn{7}{|c|}{ Growth inhibition $[\%]^{\mathrm{b}} \pm \mathrm{SD}$} \\
\hline \multicolumn{7}{|c|}{ Inhibitor + linker } \\
\hline 13 & $3 \pm 5$ & $2 \pm 5$ & $n t^{\mathrm{e}}$ & $\mathrm{nt}$ & $\mathrm{nt}$ & $\mathrm{nt}$ \\
\hline 14 & $0 \pm 4$ & $0 \pm 1$ & $\mathrm{nt}$ & $\mathrm{nt}$ & $\mathrm{nt}$ & $\mathrm{nt}$ \\
\hline 18 & $5 \pm 3$ & $5 \pm 5$ & $\mathrm{nt}$ & $\mathrm{nt}$ & $\mathrm{nt}$ & $\mathrm{nt}$ \\
\hline 20 & $5 \pm 1$ & $4 \pm 7$ & $\mathrm{nt}$ & $\mathrm{nt}$ & $\mathrm{nt}$ & $\mathrm{nt}$ \\
\hline 21 & $4 \pm 10$ & $10 \pm 1$ & $\mathrm{nt}$ & $\mathrm{nt}$ & $\mathrm{nt}$ & $\mathrm{nt}$ \\
\hline 29 & $14 \pm 1$ & $19 \pm 4$ & $\mathrm{nt}$ & $\mathrm{nt}$ & $\mathrm{nt}$ & $\mathrm{nt}$ \\
\hline \multicolumn{7}{|c|}{ Inhibitor + siderophore mimic } \\
\hline 16 & $20 \pm 4$ & $0 \pm 2$ & $13 \pm 3$ & $10 \pm 3$ & $17 \pm 10$ & $9 \pm 1$ \\
\hline 17 & $8 \pm 6$ & $1 \pm 1$ & $7 \pm 5$ & $0 \pm 5$ & $1 \pm 9$ & $7 \pm 1$ \\
\hline 34 & $26 \pm 4$ & $7 \pm 2$ & $24 \pm 5$ & $26 \pm 3$ & $29 \pm 8$ & $22 \pm 2$ \\
\hline 35 & $25 \pm 1$ & $4 \pm 5$ & $32 \pm 3$ & $71 \pm 3$ & $18 \pm 6$ & $23 \pm 1$ \\
\hline 37 & $10 \pm 3$ & $7 \pm 3$ & $3 \pm 2$ & $7 \pm 6$ & $0 \pm 5$ & $2 \pm 2$ \\
\hline 39 & $24 \pm 2$ & $0 \pm 1$ & $16 \pm 7$ & $2 \pm 1$ & $28 \pm 9$ & $10 \pm 1$ \\
\hline 44 & $23 \pm 4$ & $32 \pm 6$ & $36 \pm 6$ & $52 \pm 0$ & $36 \pm 4$ & $34 \pm 1$ \\
\hline 45 & $50 \pm 1$ & $42 \pm 2$ & $39 \pm 5$ & $57 \pm 1$ & $58 \pm 3$ & $64 \pm 1$ \\
\hline 46 & $45 \pm 2$ & $31 \pm 6$ & $32 \pm 8$ & $39 \pm 1$ & $58 \pm 1$ & $37 \pm 2$ \\
\hline 47 & $21 \pm 9$ & $31 \pm 2$ & $26 \pm 8$ & $55 \pm 1$ & $47 \pm 2$ & $44 \pm 3$ \\
\hline
\end{tabular}

${ }^{a}$ Minimum inhibitory concentration that inhibits growth of the bacteria by $\geq 90 \%$

${ }^{b}$ Inhibition of growth at $50 \mu \mathrm{M}$ of the tested compound relative to the control 
${ }^{c}$ Cefiderocol used as positive control

${ }^{d}$ Ceftazidime used as negative control

${ }^{e}$ not tested 


\section{3. ${ }^{1} \mathrm{H}$ AND ${ }^{13} \mathrm{C}$ NMR SPECTRA OF THE REPRESENTATIVE COMPOUNDS}

(S)-N-(2-(5-(Benzyloxy)-4-oxo-4H-pyran-2-carboxamido)-4,5,6,7-tetrahydrobenzo[d]thiazol-6-yl)-4,5-dibromo-1H-pyrrole-2-carboxamide (15). $1 \mathrm{H} \mathrm{NMR} \mathrm{(400} \mathrm{MHz,} \mathrm{DMSO-d6)}$

峾
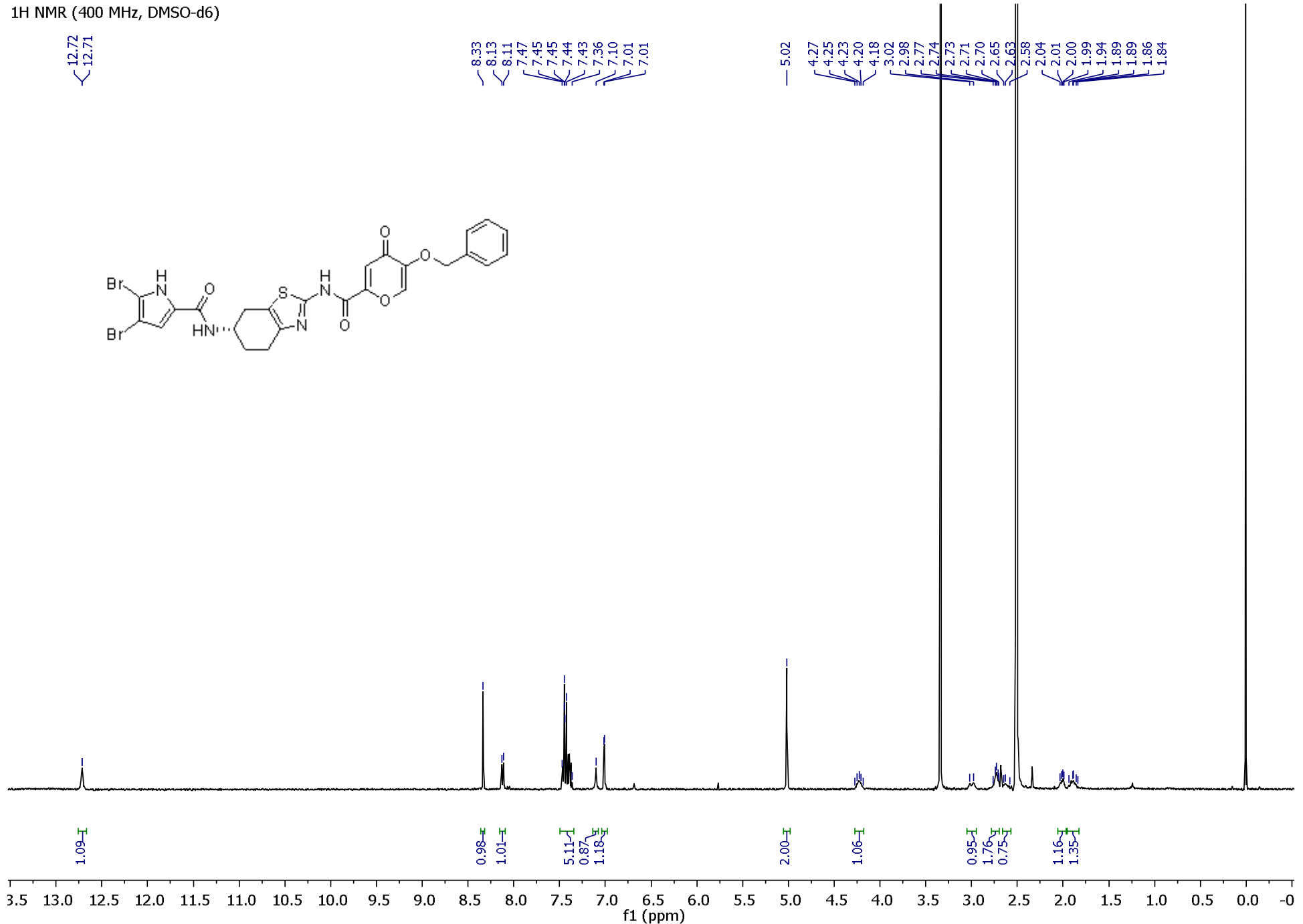
13C NMR (100 MHz, DMSO-d6)

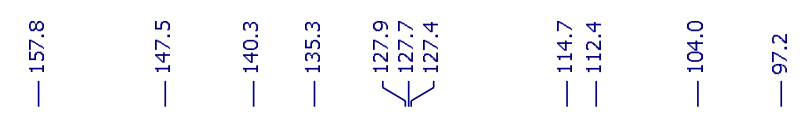

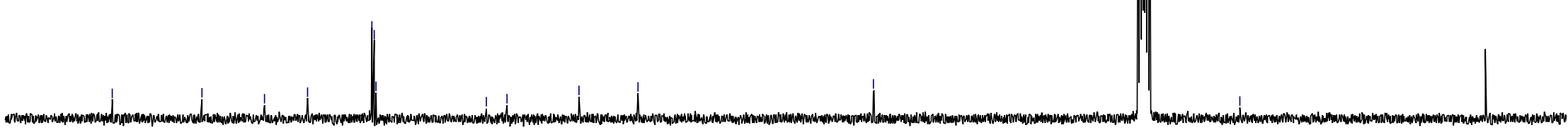


(S)-4,5-Dibromo-N-(2-(5-hydroxy-4-oxo-4H-pyran-2-carboxamido)-4,5,6,7-tetrahydrobenzo[d]thiazol-6-yl)-1H-pyrrole-2-carboxamide (16). 1H NMR (400 MHz, DMSO-d6)

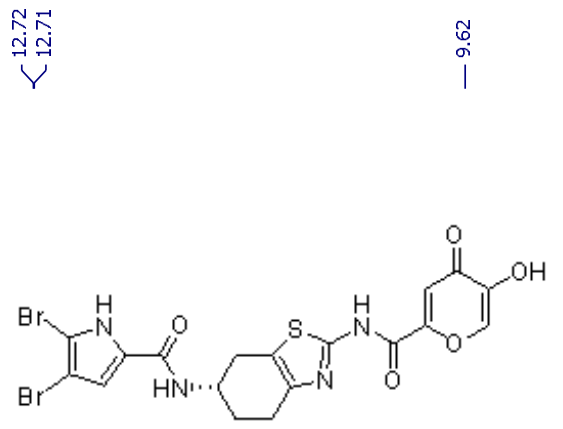

*EtOAc

\# signal partially overlapped with EtOAc
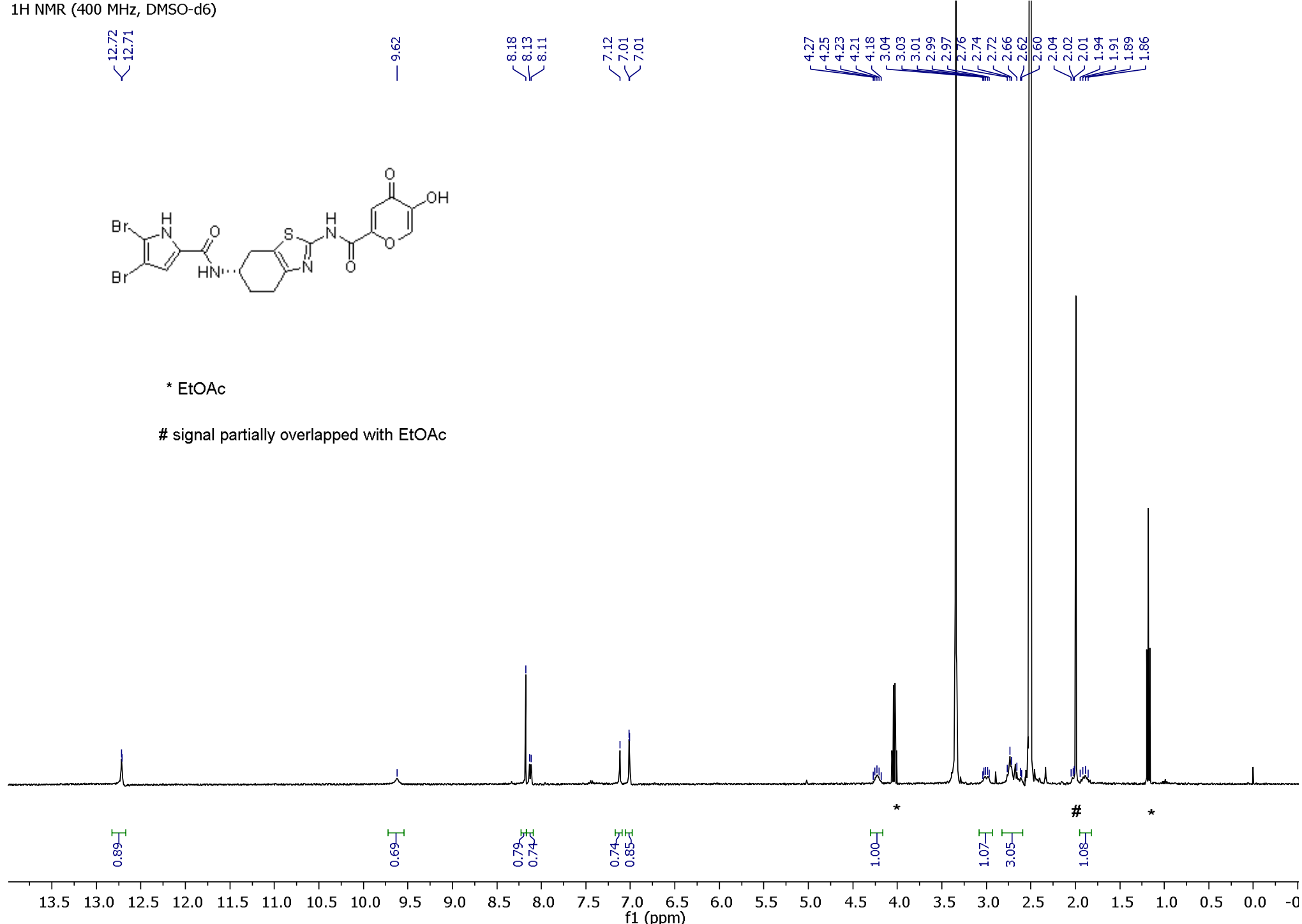
13C NMR (100 MHz, DMSO-d6)

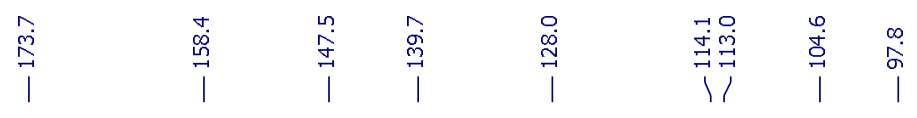

* EtOAc

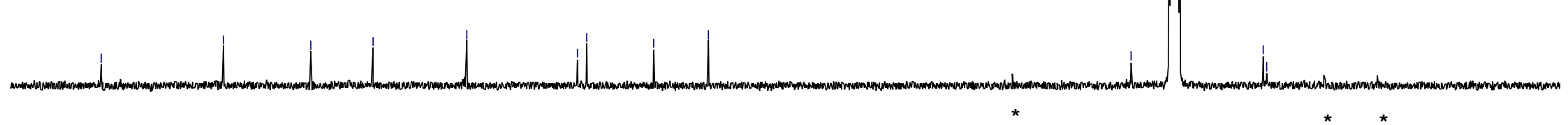


(S)-3,4-Dichloro-N-(2-(5-hydroxy-4-oxo-4H-pyran-2-carboxamido)-4,5,6,7-tetrahydrobenzo[d]thiazol-6-yl)-5-methyl-1H-pyrrole-2-carboxamide (17). 1H NMR (400 MHz, DMSO-d6)

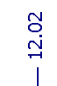

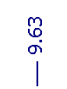
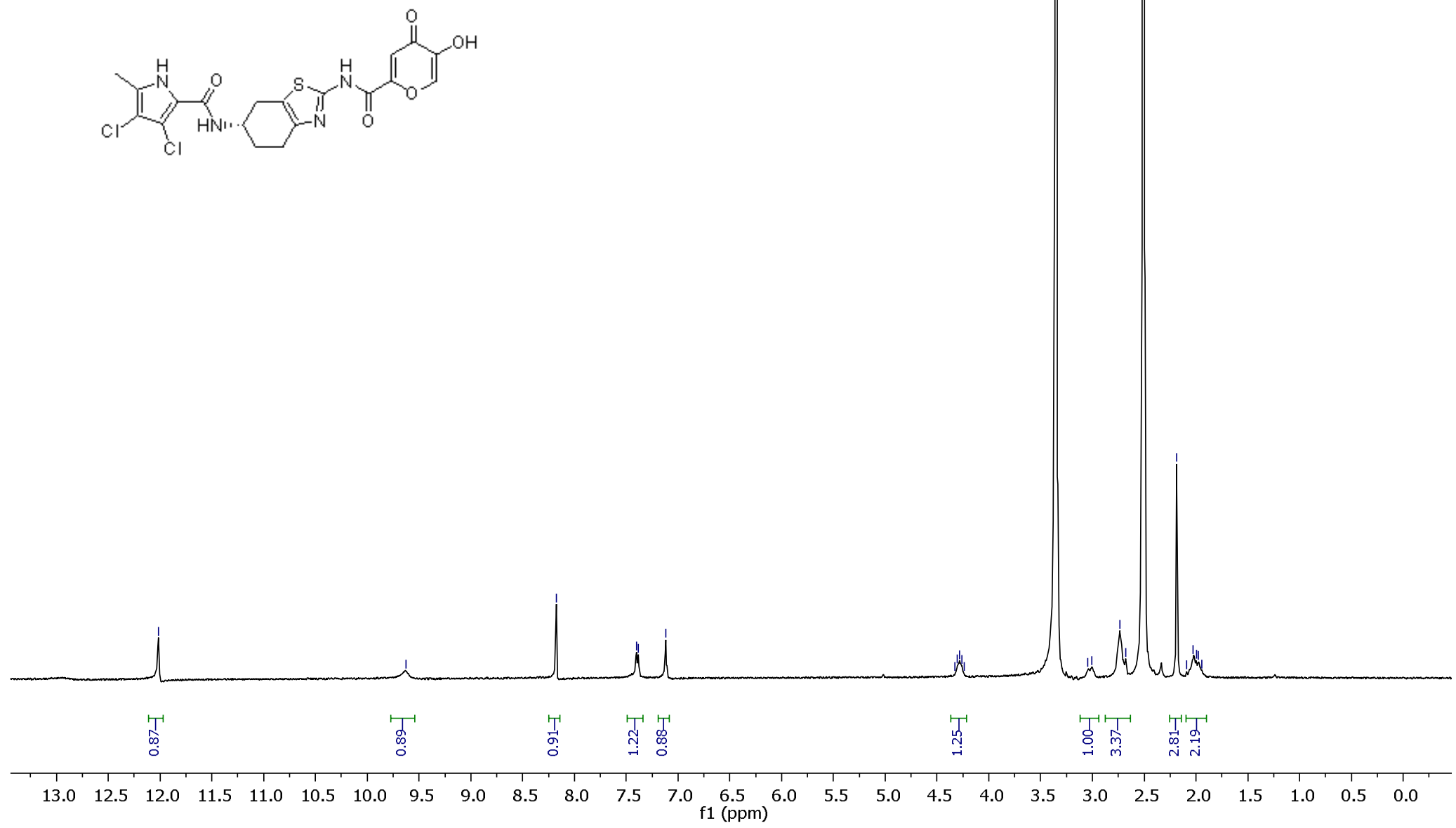
13C NMR (100 MHz, DMSO-d6)

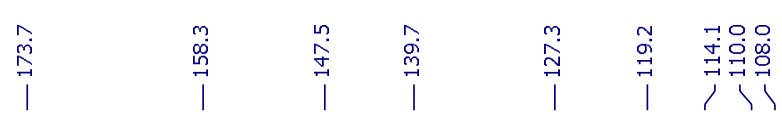
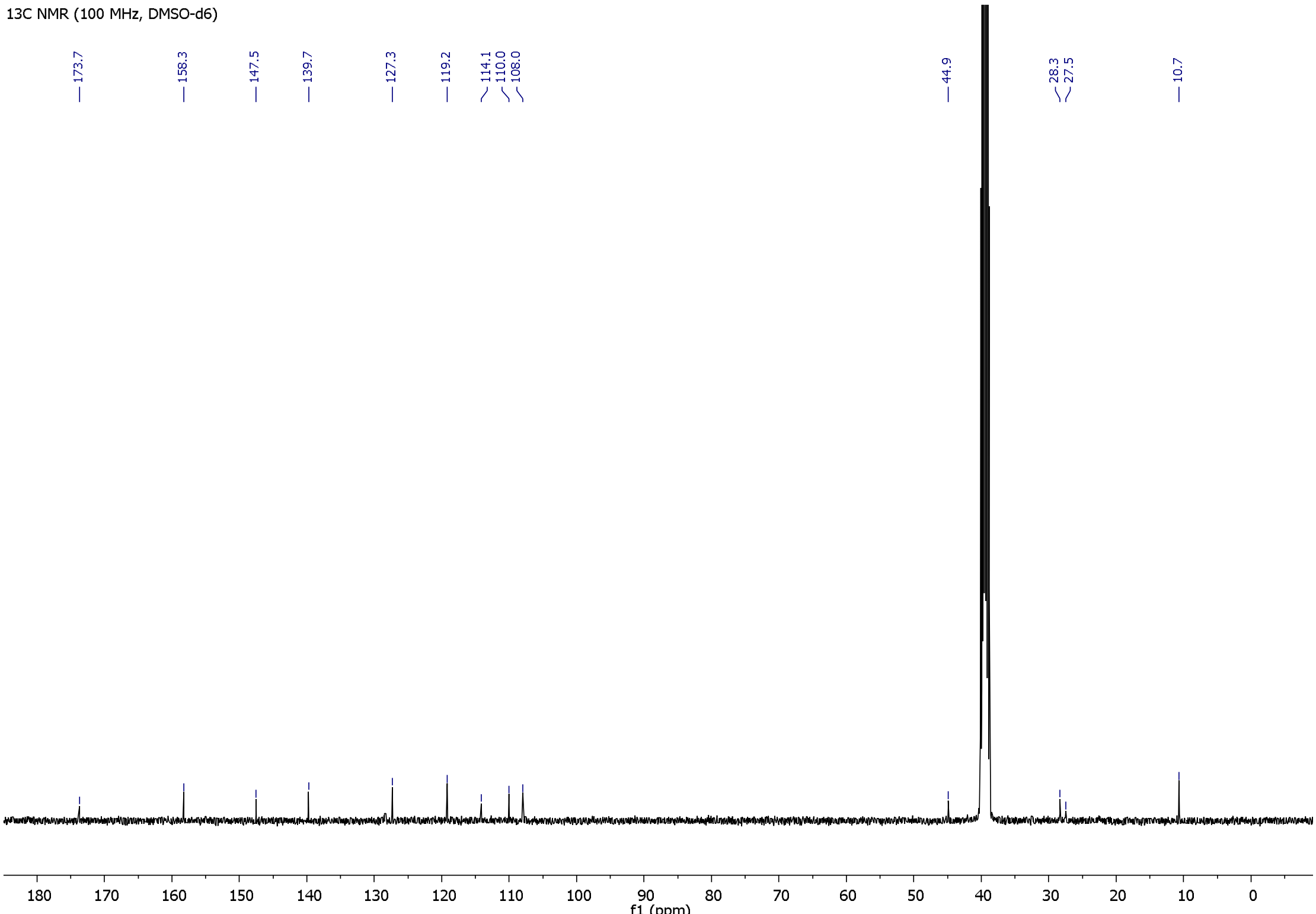
(S)-N-(2-(2-Acetamidoacetamido)-4,5,6,7-tetrahydrobenzo[d] thiazol-6-yl)-4,5-dibromo-1H-pyrrole-2-carboxamide (18).

1H NMR (400 MHz, DMSO-d6)

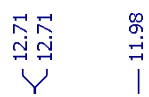
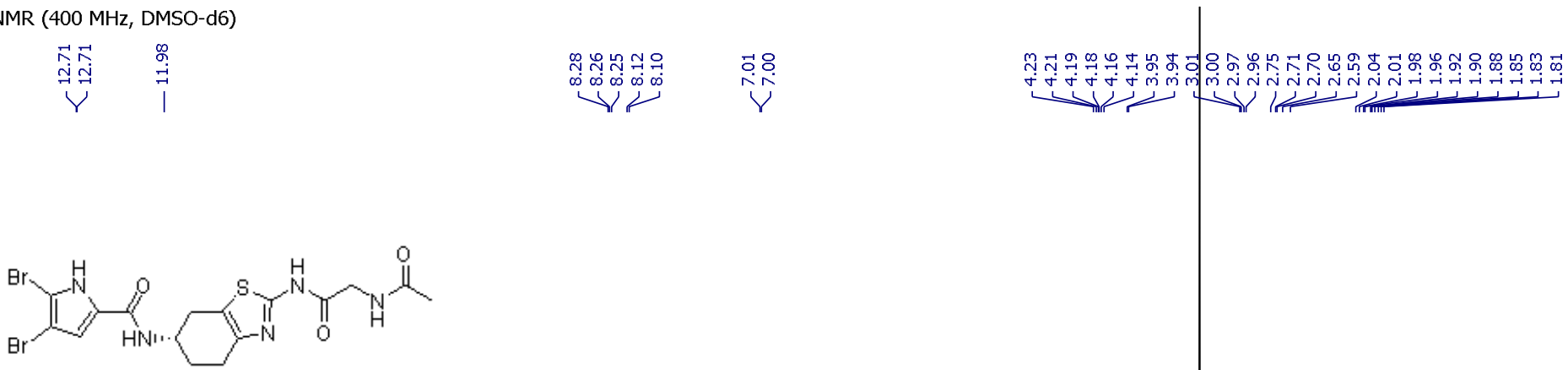

* EtOAc

\# signal partially overlapped with EtOAc

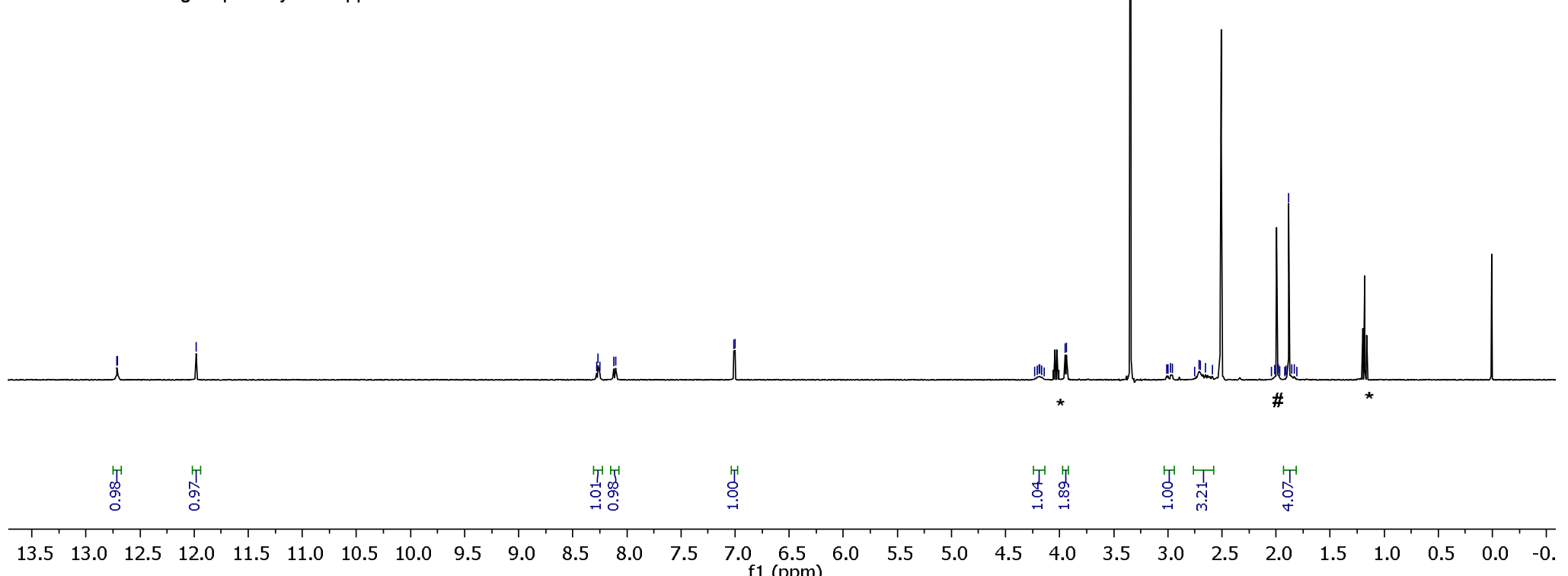


13C NMR (100 MHz, DMSO-d6)

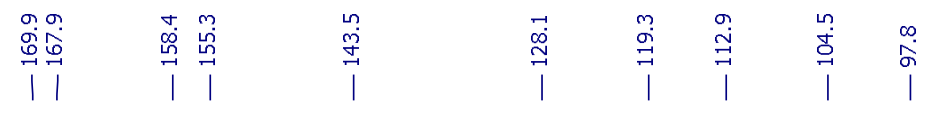

${ }^{*}$ EtOAc

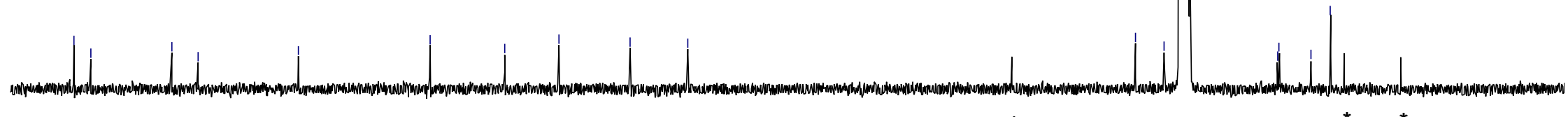

\begin{tabular}{|c|c|c|c|c|c|c|c|c|c|c|c|c|c|c|c|c|}
\hline 170 & 160 & 150 & 140 & 130 & 120 & 110 & 100 & $\begin{array}{l}90 \\
\mathrm{f} 1(\mathrm{ppm})\end{array}$ & 70 & 60 & 50 & 40 & 30 & 20 & 10 & 0 \\
\hline
\end{tabular}


N-((S)-2-((S)-2-Acetamidopropanamido)-4,5,6,7-tetrahydrobenzo[d]thiazol-6-yl)-4,5-dibromo-1H-pyrrole-2-carboxamide (20).

1H NMR (400 MHz, DMSO-d6)

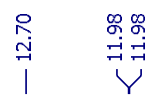

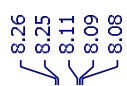

ìn
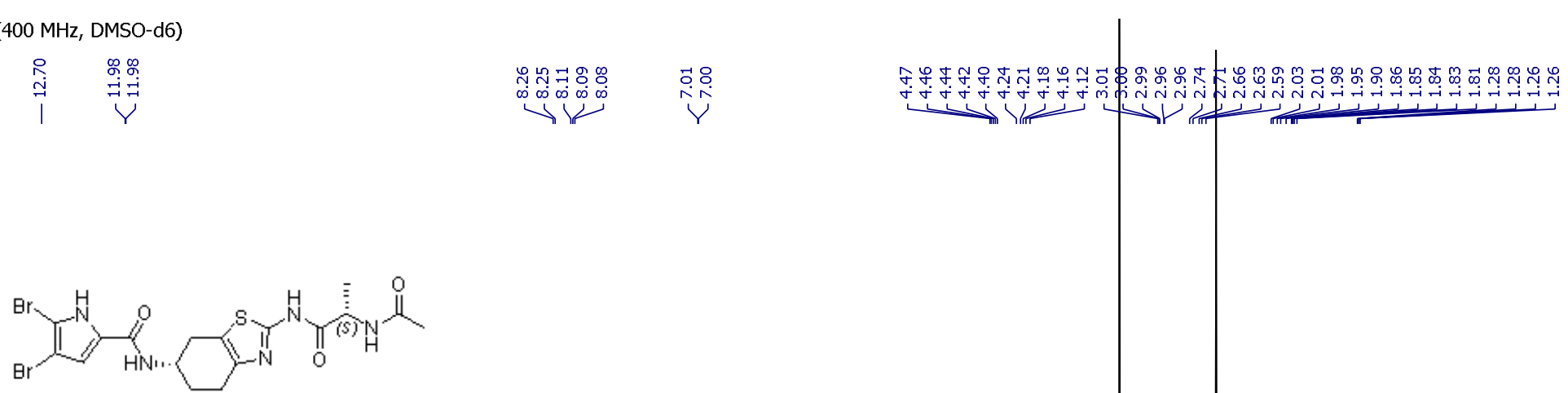

* EtOAc

\# signal partially overlapped with EtOAc

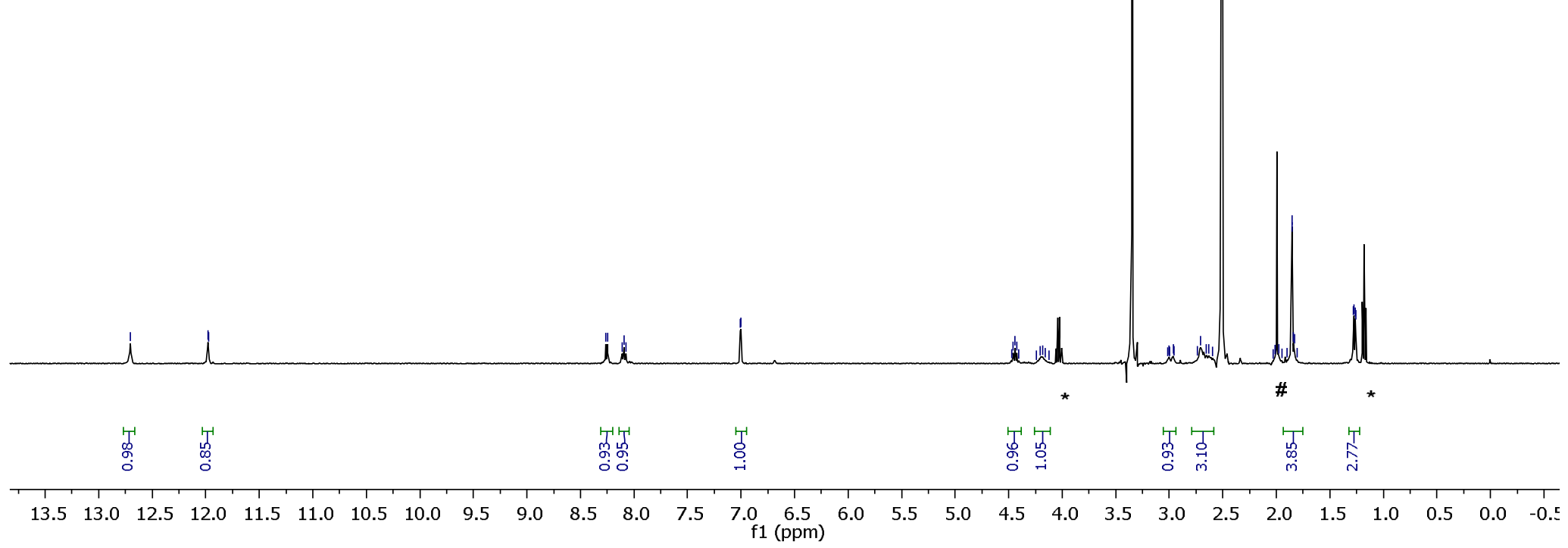


13C NMR (100 MHz, DMSO-d6)

年我

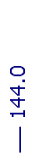

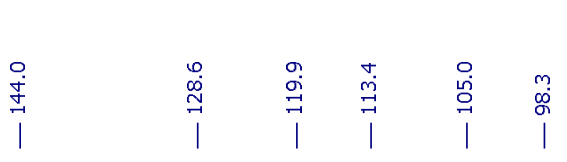

$\stackrel{\substack{c \\ \infty}}{\infty}$
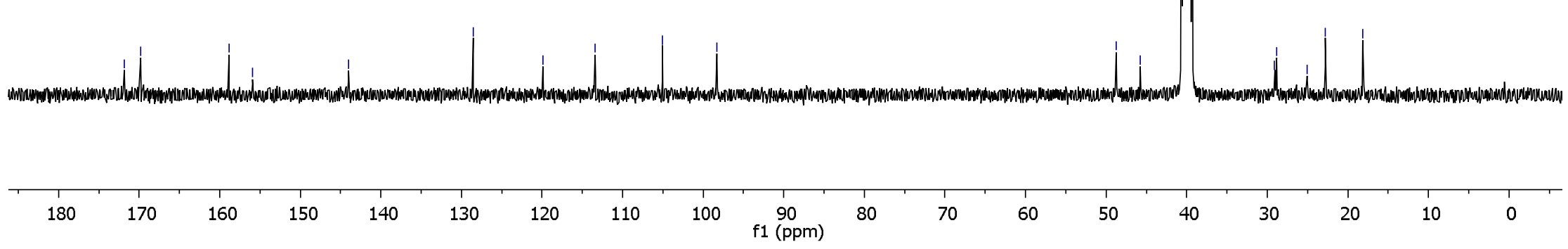

20 
Tert-butyl (S)-(2-((6-(3,4-dichloro-5-methyl-1H-pyrrole-2-carboxamido)-4,5,6,7-tetrahydrobenzo[d]thiazol-2-yl)amino)-2-oxoethyl)carbamate (25). 1H NMR (400 MHz, DMSO-d6)

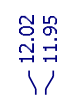

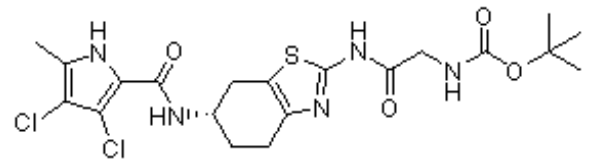

* EtOAc

\# signal partially overlapped with EtOAc

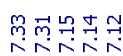

viv

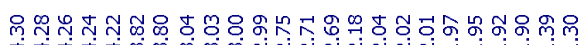

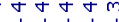

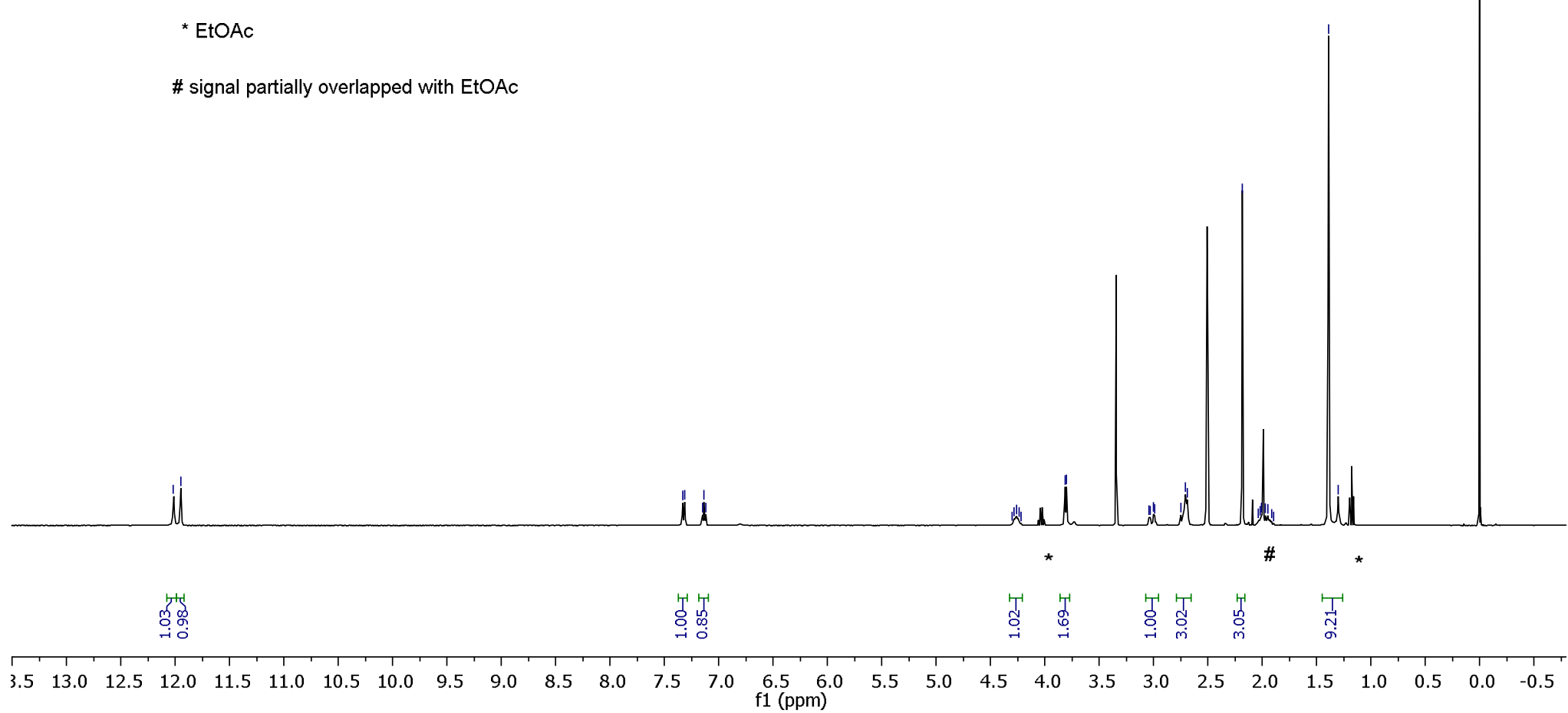


13C NMR (100 MHz, DMSO-d6) |

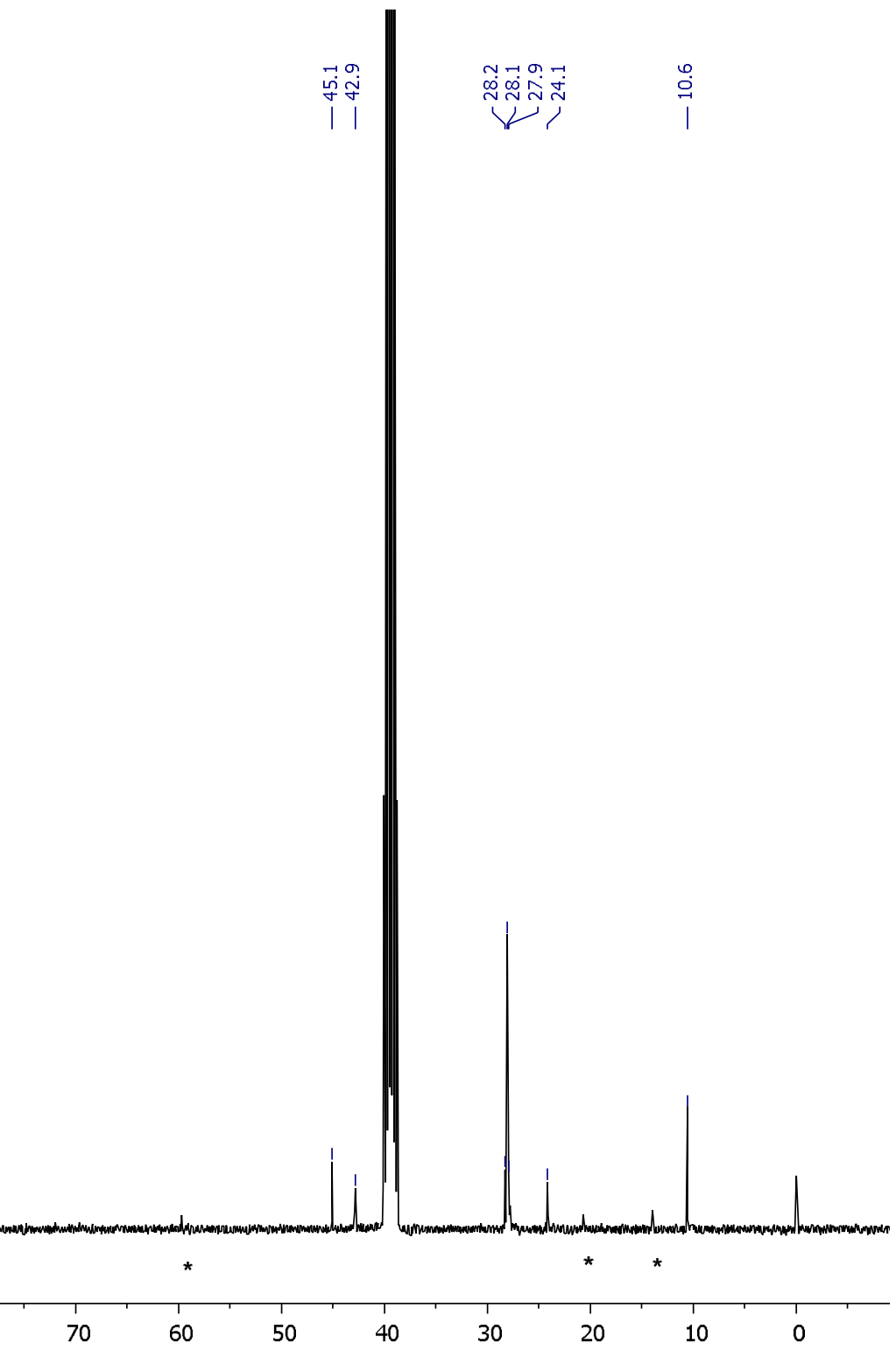

$80 \quad 170 \quad 160$
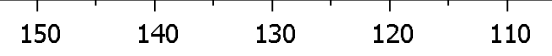

100 
Tert-butyl ((S)-1-(((S)-6-(3,4-dichloro-5-methyl-1H-pyrrole-2-carboxamido)-4,5,6,7-tetrahydrobenzo[d]thiazol-2-yl)amino)-3-methyl-1-oxobutan-2-yl)carbamate (27).

$1 \mathrm{H}$ NMR (400 MHz, DMSO-d6)<smiles></smiles>

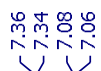
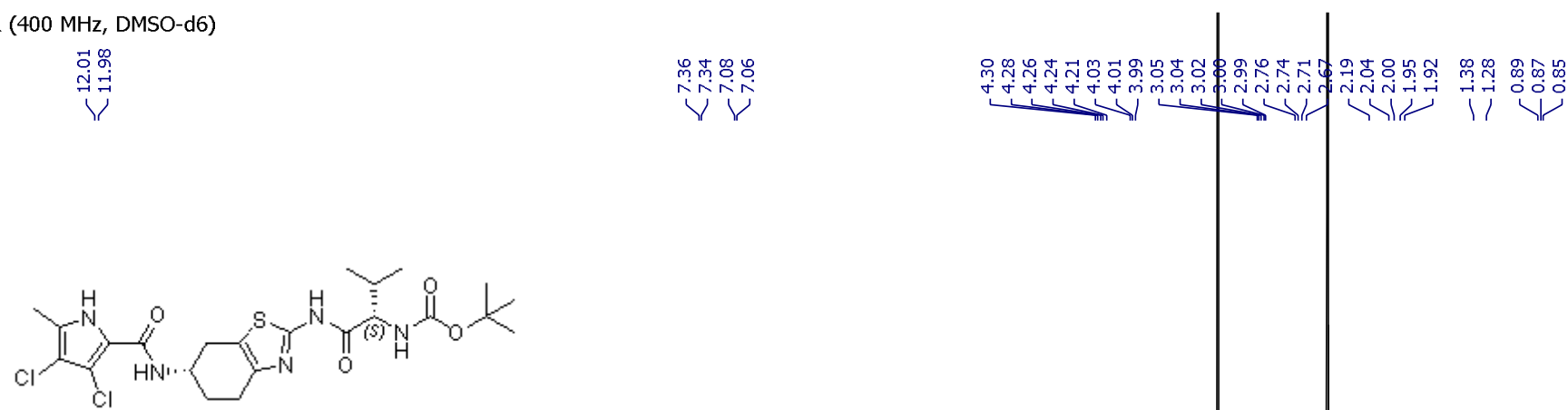

\section{* acetone}

\# dichloromethane

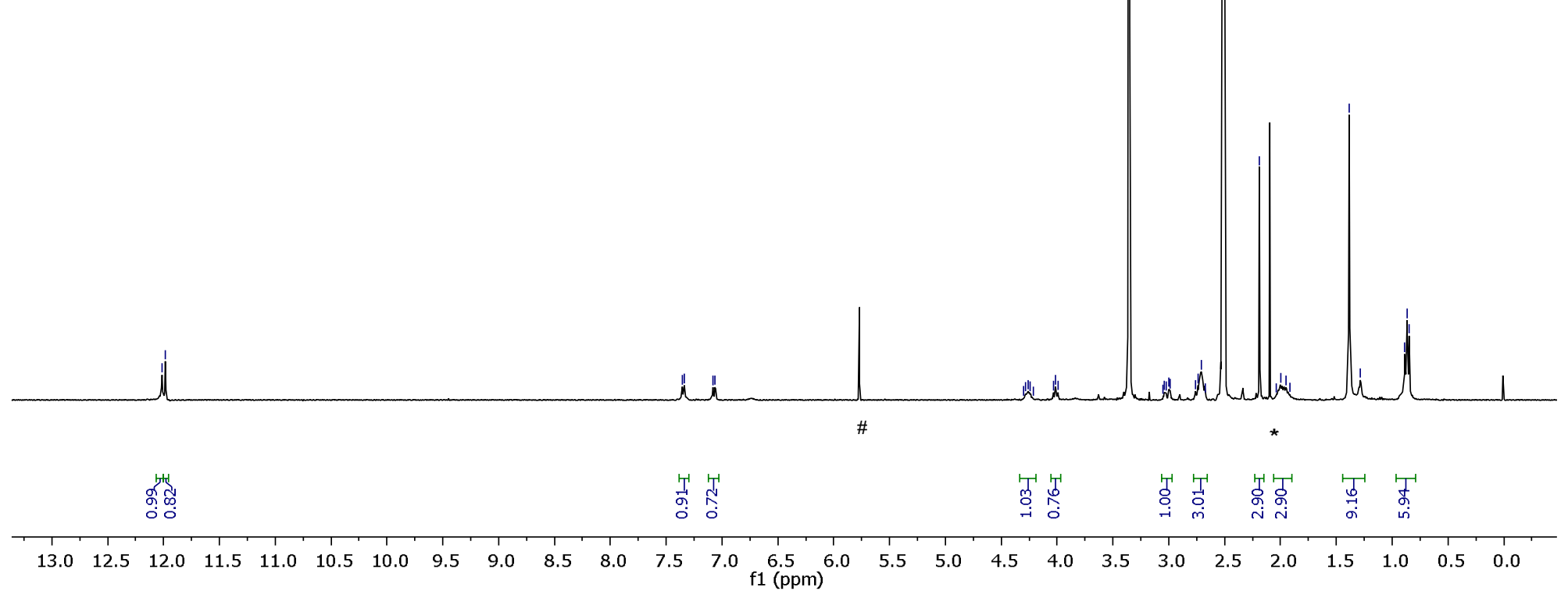




$$
1
$$


(S)-2-((6-(3,4-Dichloro-5-methyl-1H-pyrrole-2-carboxamido)-4,5,6,7-tetrahydrobenzo[d]thiazol-2-yl)amino)-2-oxoethan-1-aminium chloride (30). 1H NMR (400 MHz, DMSO-d6)
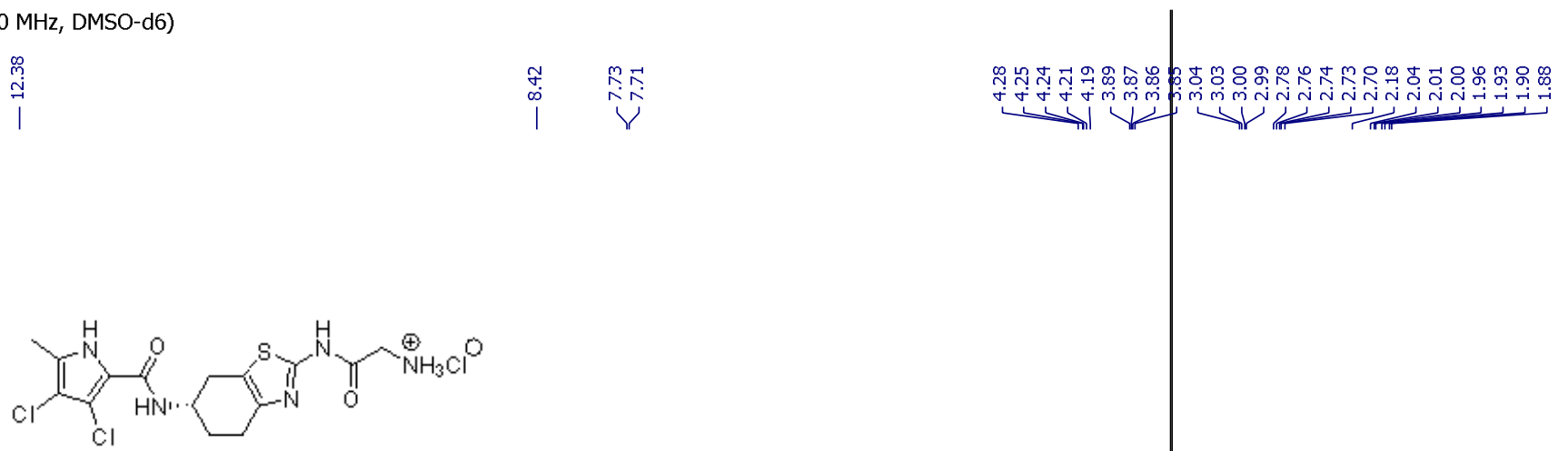

*1,4-dioxane

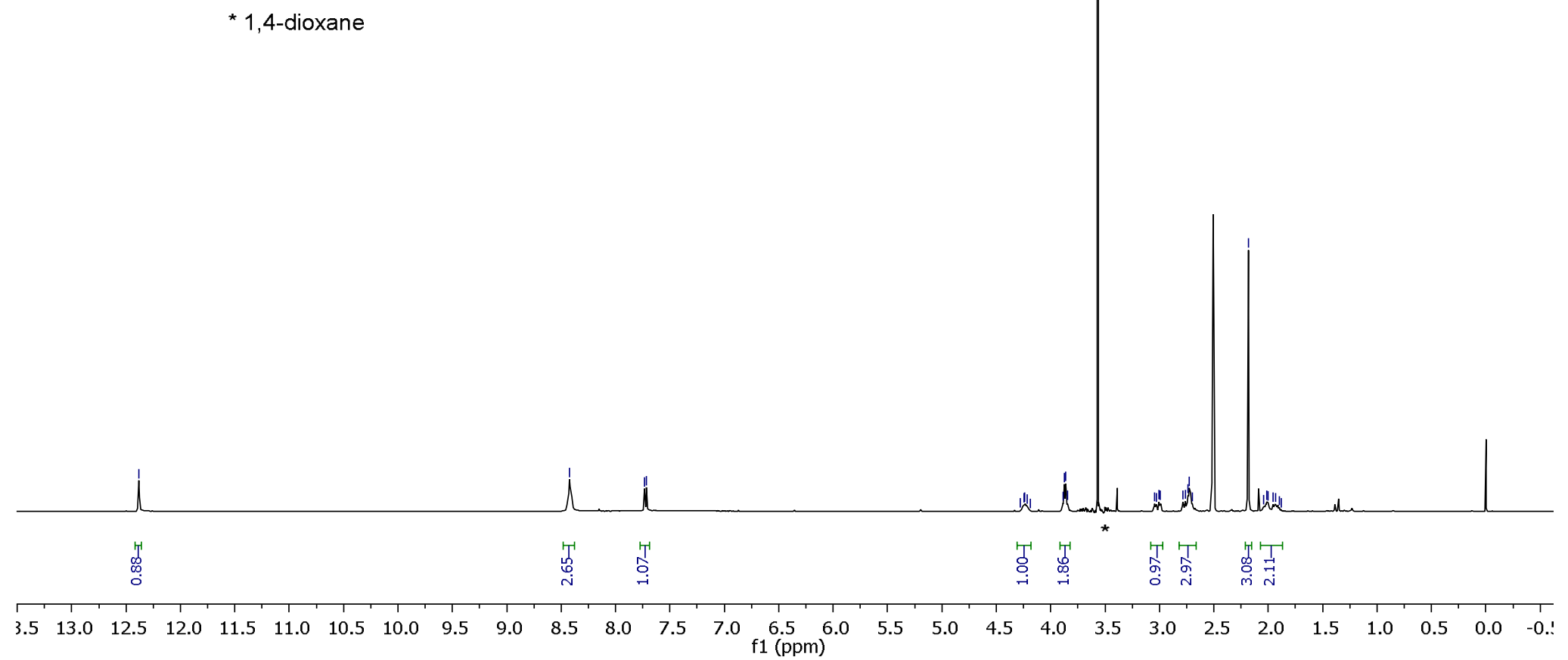


$13 \mathrm{C}$ NMR ( $100 \mathrm{MHz}$, DMSO-d6)

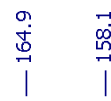

*1,4-dioxane

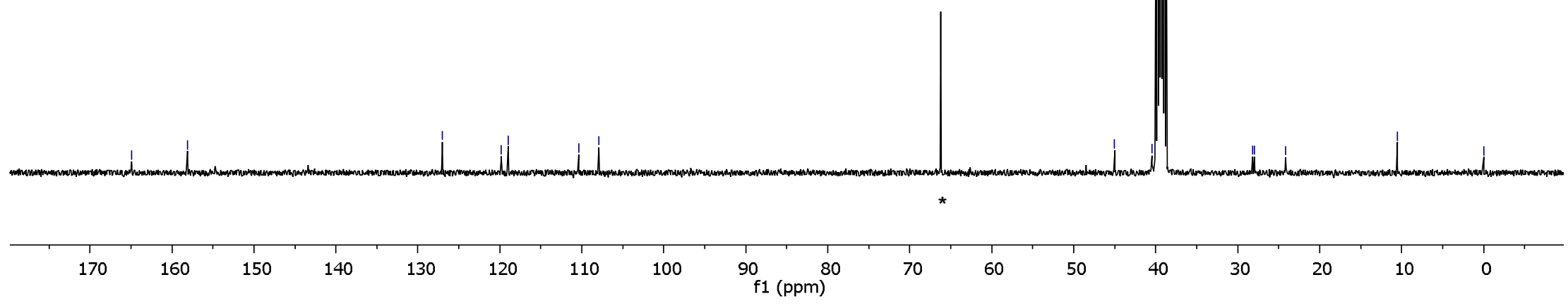


(S)-3-((6-(3,4-Dichloro-5-methyl-1H-pyrrole-2-carboxamido)-4,5,6,7-tetrahydrobenzo[d]thiazol-2-yl)amino)-3-oxopropan-1-aminium chloride (33). $1 \mathrm{H}$ NMR (400 MHz, DMSO-d6)

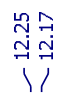

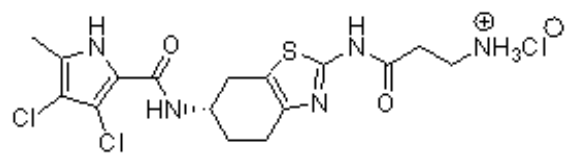

*1,4-dioxane

\# diethyl ether

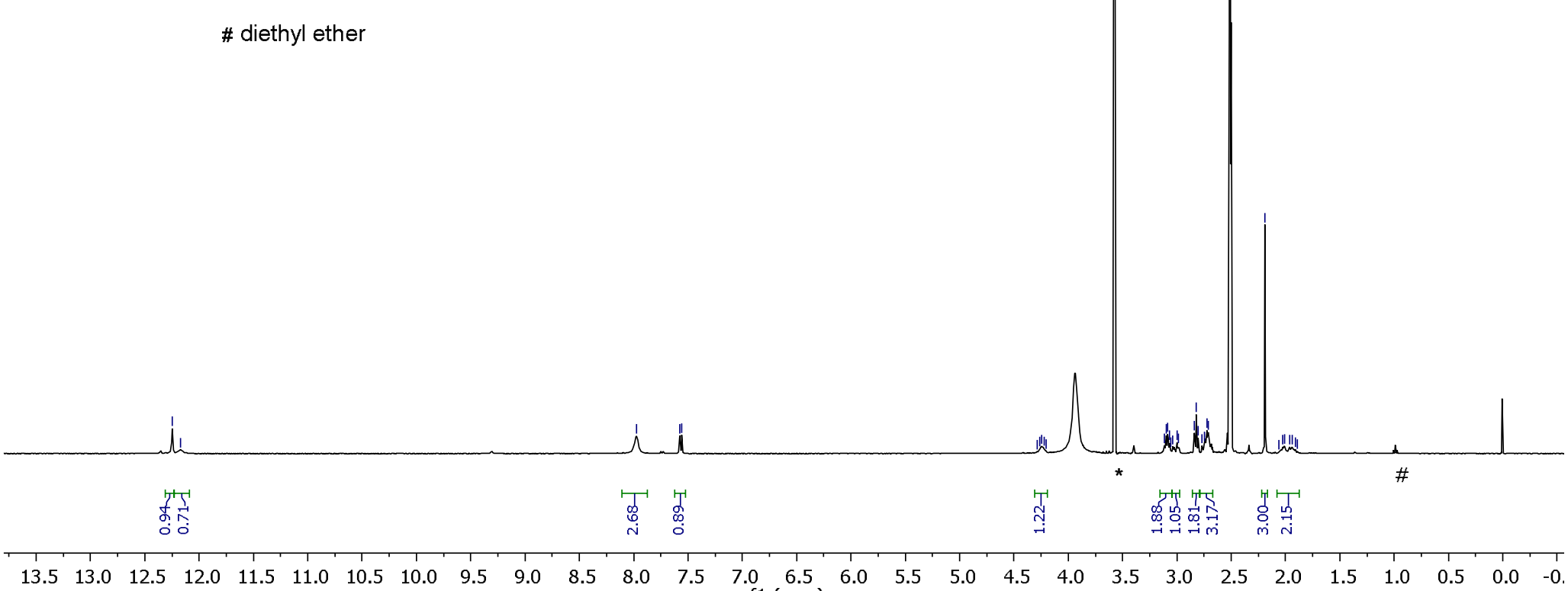

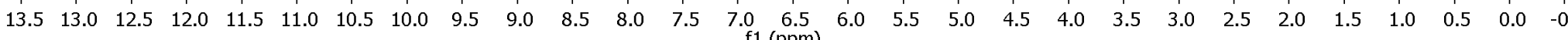


13C NMR (100 MHz, DMSO-d6)

$$
\text { 点 }
$$

* 1,4-dioxane

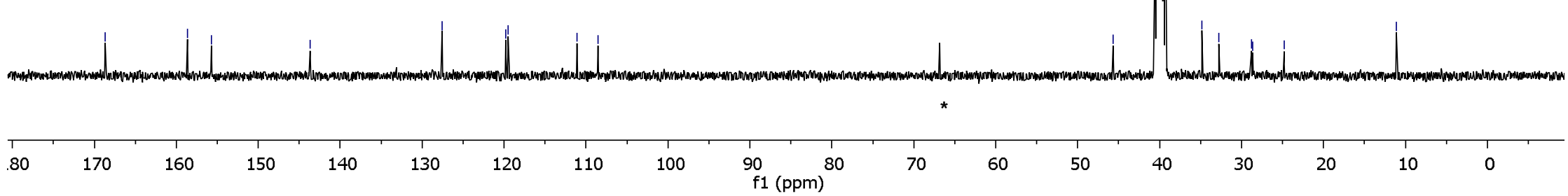


(S)-3,4-Dichloro-N-(2-(2-(3,4-dihydroxybenzamido)acetamido)-4,5,6,7-tetrahydrobenzo[d]thiazol-6-yl)-5-methyl-1H-pyrrole-2-carboxamide (34). 1H NMR (400 MHz, DMSO-d6)
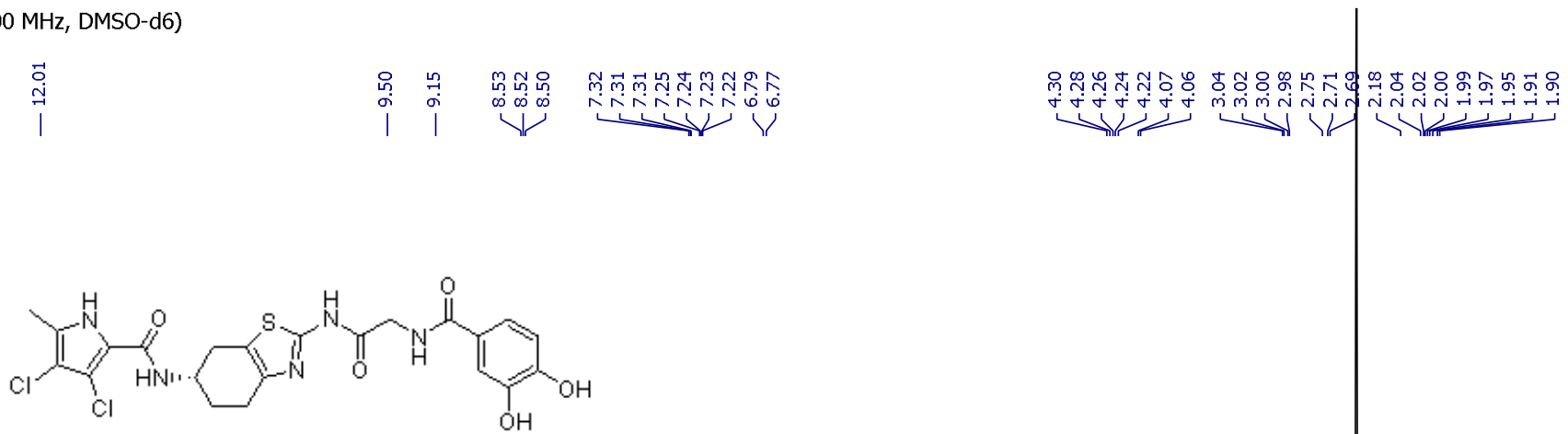

*DMF

\# signal partially overlapped with DMF

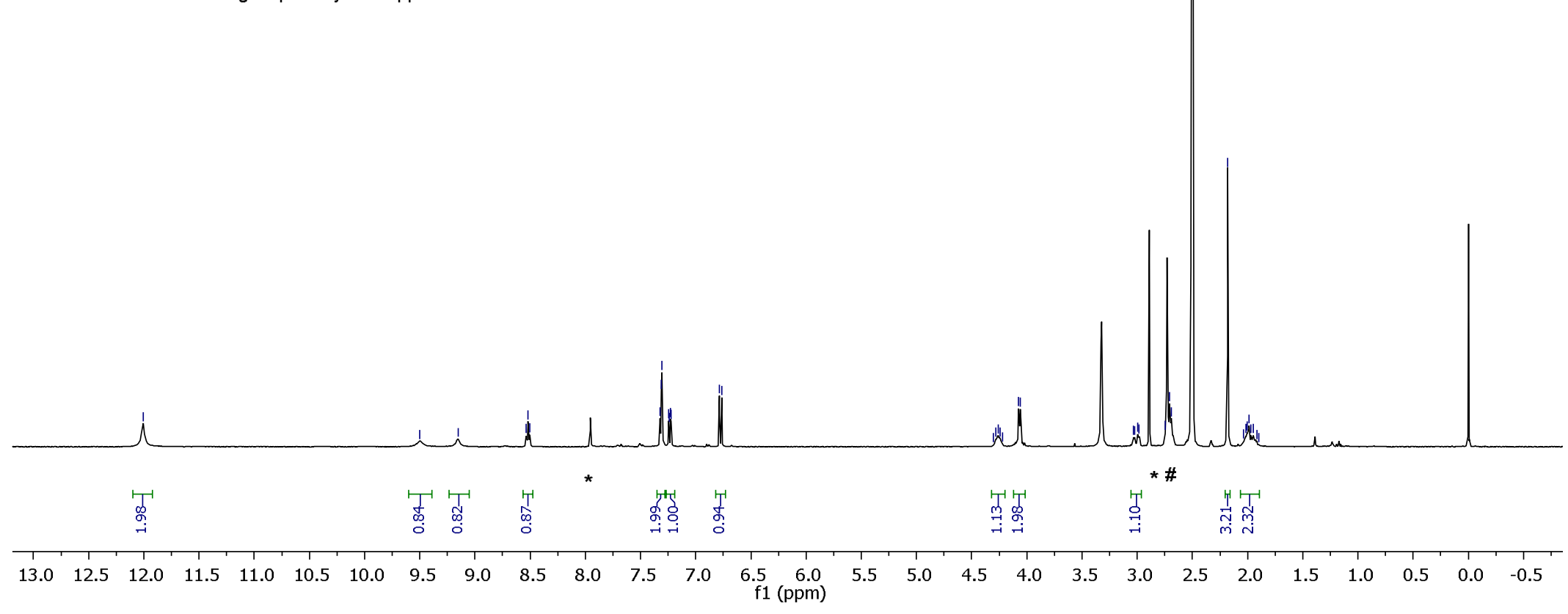


13C NMR (100 MHz, DMSO-d6)

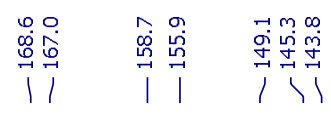

|

* DMF
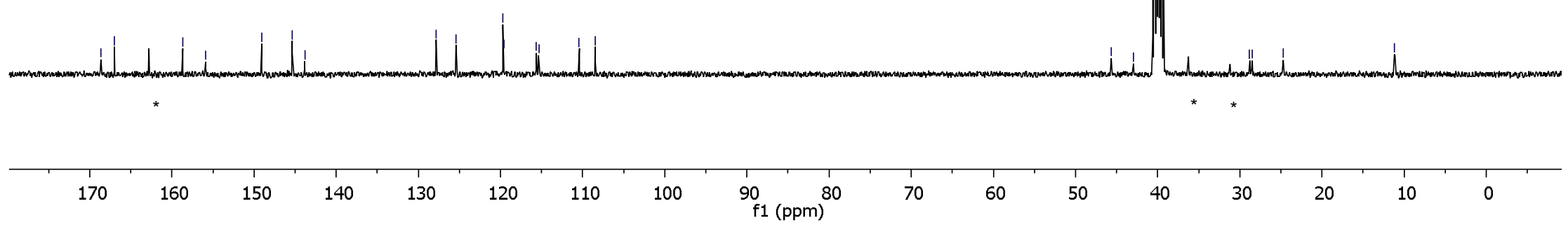

30 
(S)-3,4-Dichloro-N-(2-(2-(2,3-dihydroxybenzamido)acetamido)-4,5,6,7-tetrahydrobenzo[d]thiazol-6-yl)-5-methyl-1H-pyrrole-2-carboxamide (35). 1H NMR (400 MHz, DMSO-d6)

월워
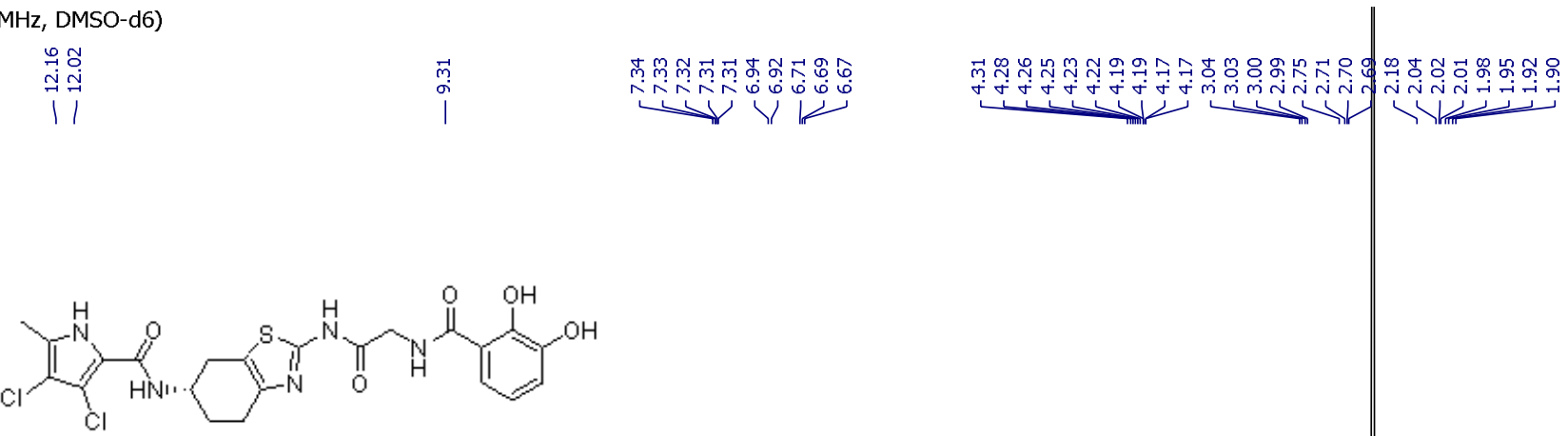

${ }^{*}$ EtOAc

\$DF

\# signal partially overlapped with EtOAc

$\S$ signal partially overlapped with DMF

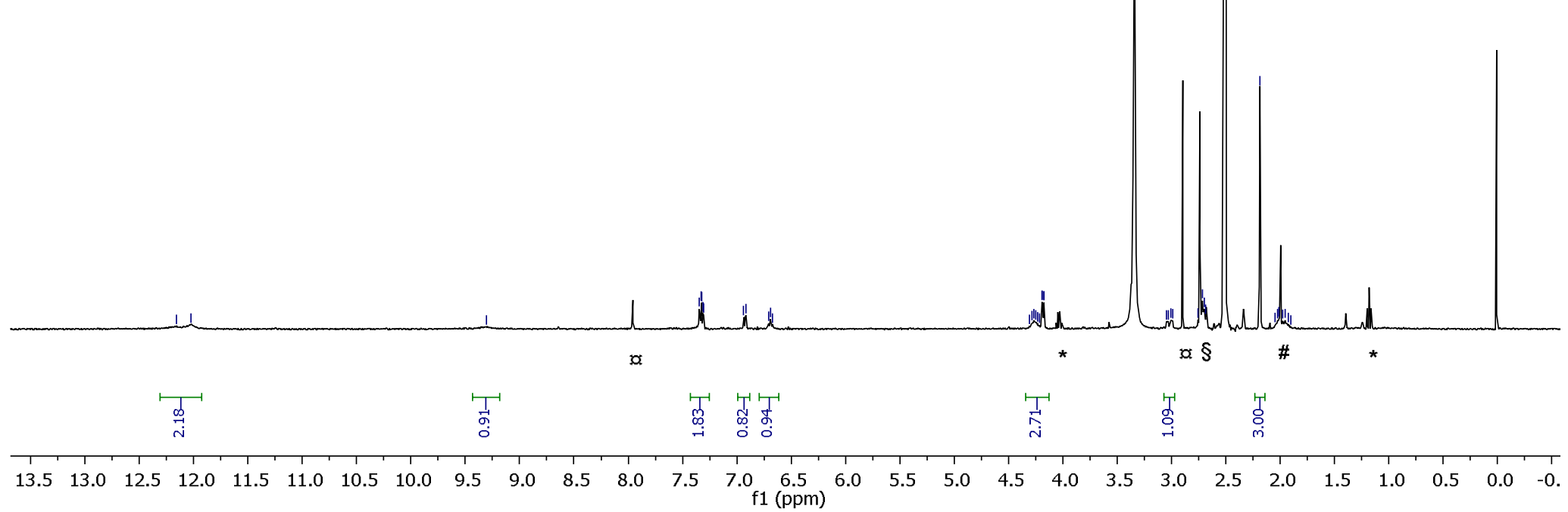


13C NMR (100 MHz, DMSO-d6) |

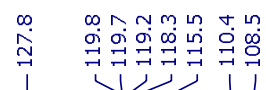

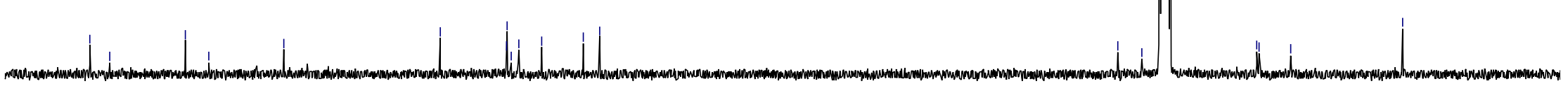
${ }_{80} \quad 170$ $160 \quad 150$ 
(S)-3,4-Dichloro-N-(2-(2-(5-hydroxy-4-oxo-4H-pyran-2-carboxamido)acetamido)-4,5,6,7-tetrahydrobenzo[d]thiazol-6-yl)-5-methyl-1H-pyrrole-2-carboxamide (39).

$1 \mathrm{H} \mathrm{NMR} \mathrm{(400} \mathrm{MHz,} \mathrm{DMSO-d6)}$

กैช

ㄱำ

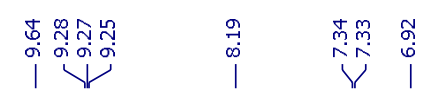
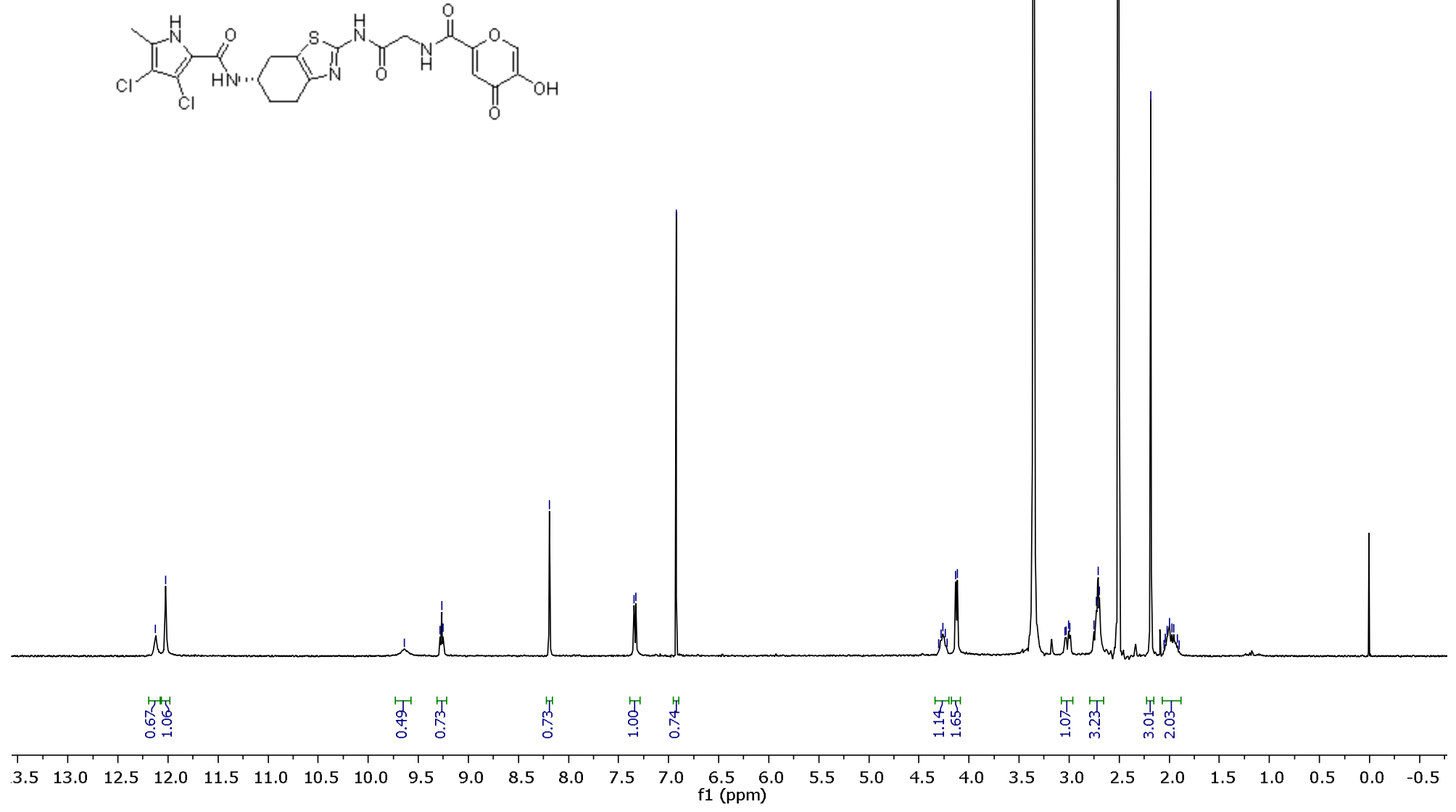
13C NMR (100 MHz, DMSO-d6)

| |

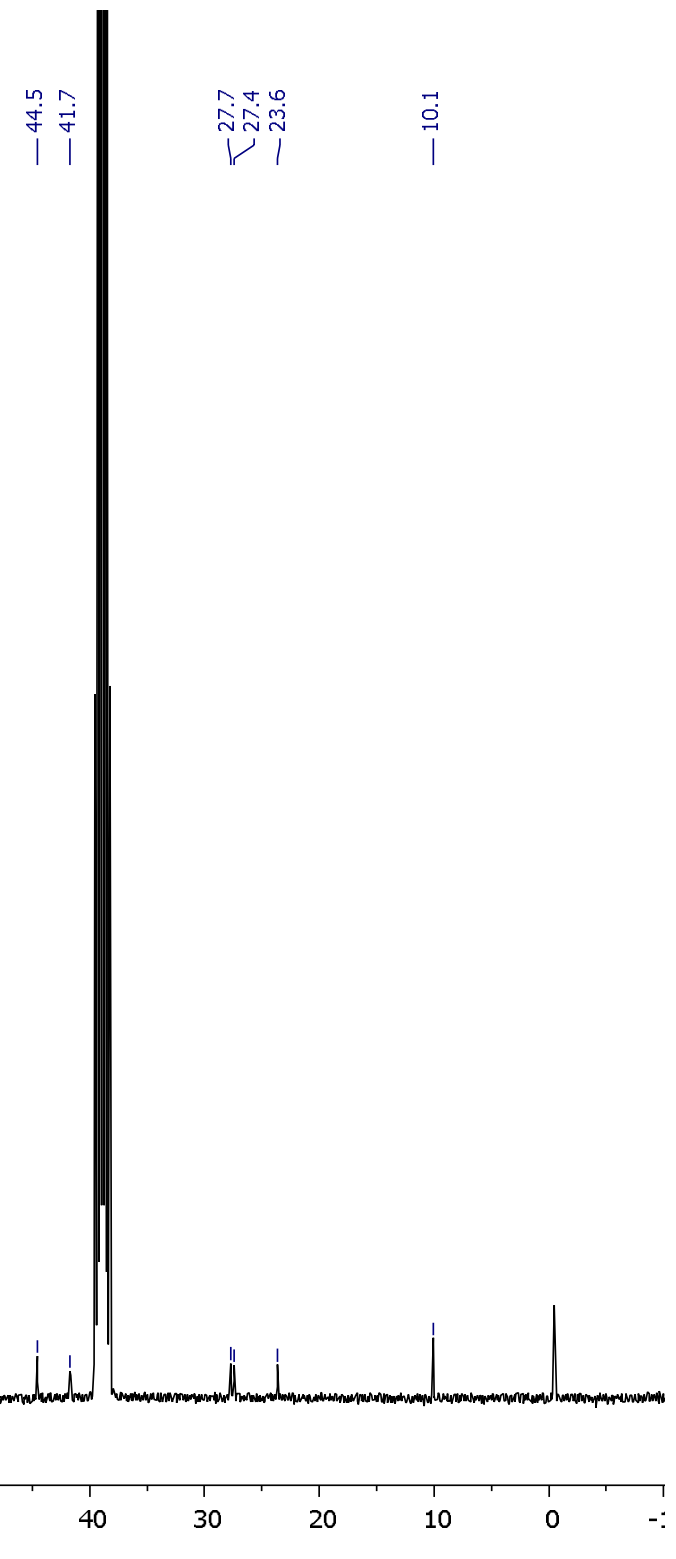


(S)-N-(3-((6-(3,4-Dichloro-5-methyl-1H-pyrrole-2-carboxamido)-4,5,6,7-tetrahydrobenzo[d]thiazol-2-yl)amino)-3-oxopropyl)-5-((4-methoxybenzyl)oxy)-4-oxo1,4-dihydropyridine-2-carboxamide (43).

$1 \mathrm{H} \mathrm{NMR} \mathrm{(400} \mathrm{MHz,} \mathrm{DMSO-d6)}$

तु

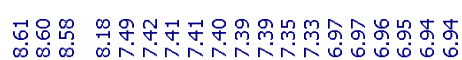
$\underbrace{\infty}$

年

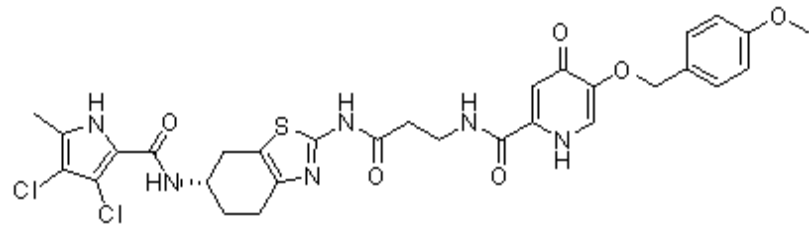

${ }^{*}$ EtOAc

\# signal partially overlapped with EtOAc

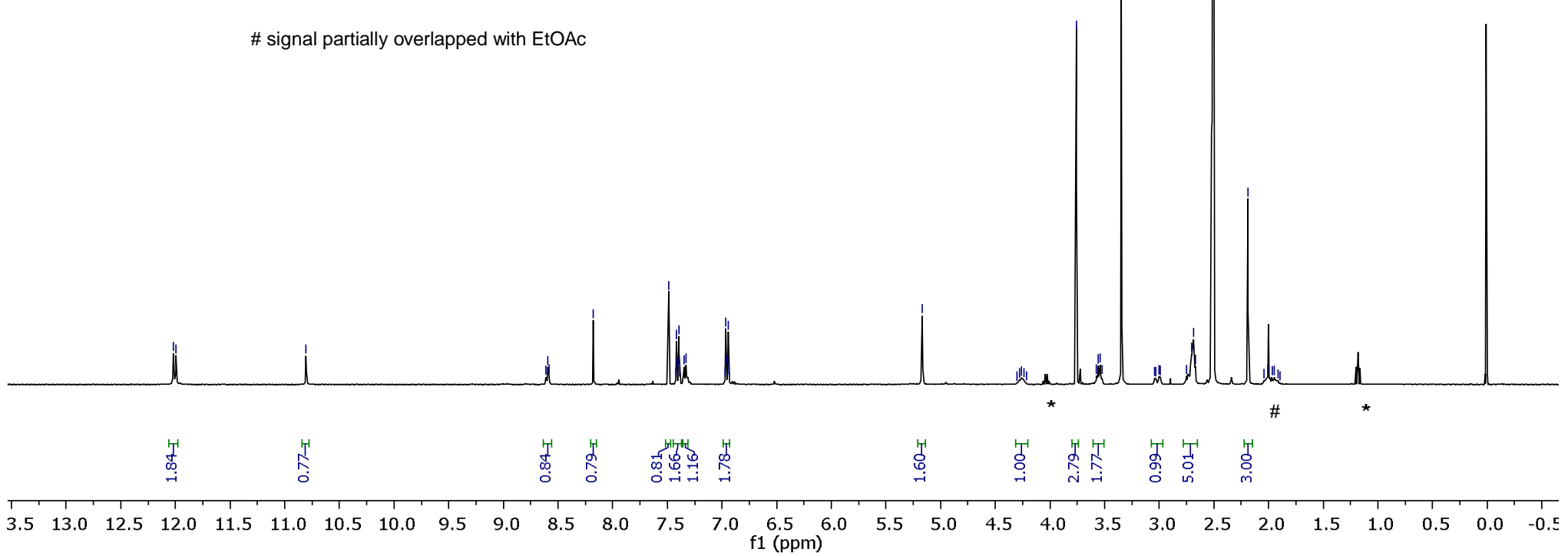


13C NMR (100 MHz, DMSO-d6)

I |

:

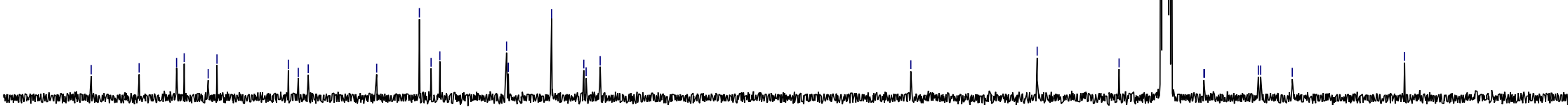

$80 \quad 170$

160

150

140

$130 \quad 120$

110

100

$90 \quad 80$ 
(S)-N-(2-((6-(3,4-Dichloro-5-methyl-1H-pyrrole-2-carboxamido)-4,5,6,7-tetrahydrobenzo[d]thiazol-2-yl)amino)-2-oxoethyl)-5-hydroxy-4-oxo-1,4dihydropyridine-2-carboxamide (44).

1H NMR (400 MHz, DMSO-d6)

$$
\stackrel{\overrightarrow{1}}{\stackrel{1}{1}}
$$

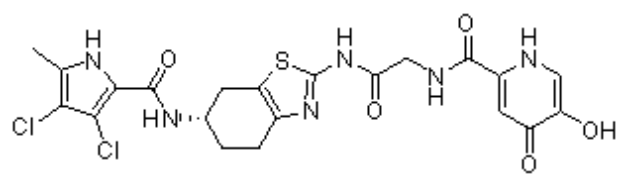

* EtOAc

\# signal partially overlapped with water$$
\mathrm{OH}
$$

i

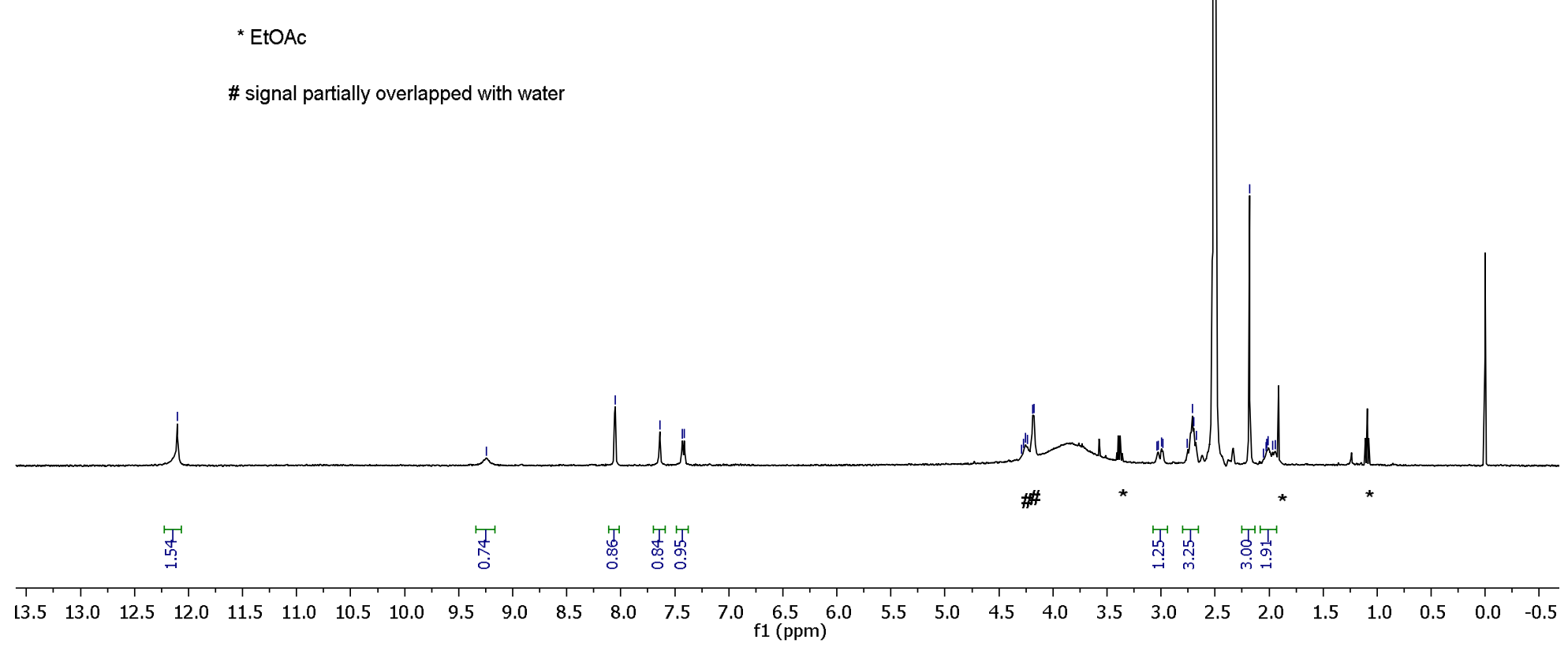


13C NMR (100 MHz, DMSO-d6)

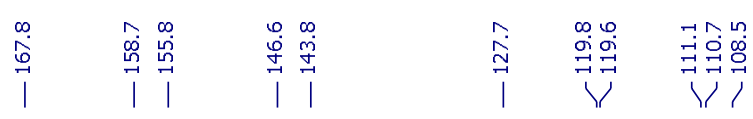

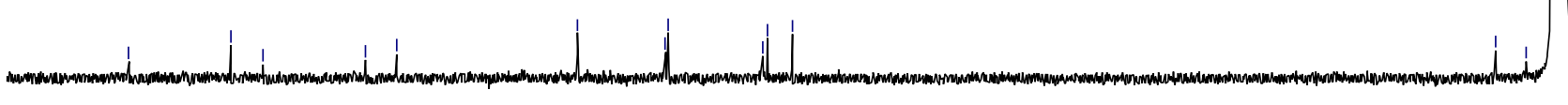
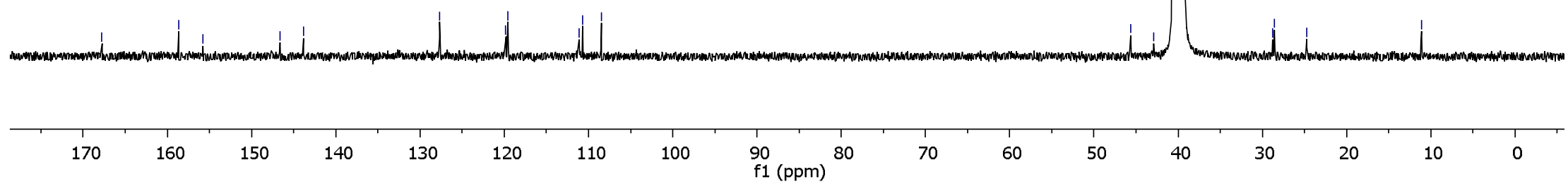
N-((S)-1-(((S)-6-(3,4-Dichloro-5-methyl-1H-pyrrole-2-carboxamido)-4,5,6,7-tetrahydrobenzo[d]thiazol-2-yl)amino)-1-oxopropan-2-yl)-5-hydroxy-4-oxo-1,4dihydropyridine-2-carboxamide (45).

$1 \mathrm{H} \mathrm{NMR} \mathrm{(400} \mathrm{MHz,} \mathrm{DMSO-d6)}$

号通
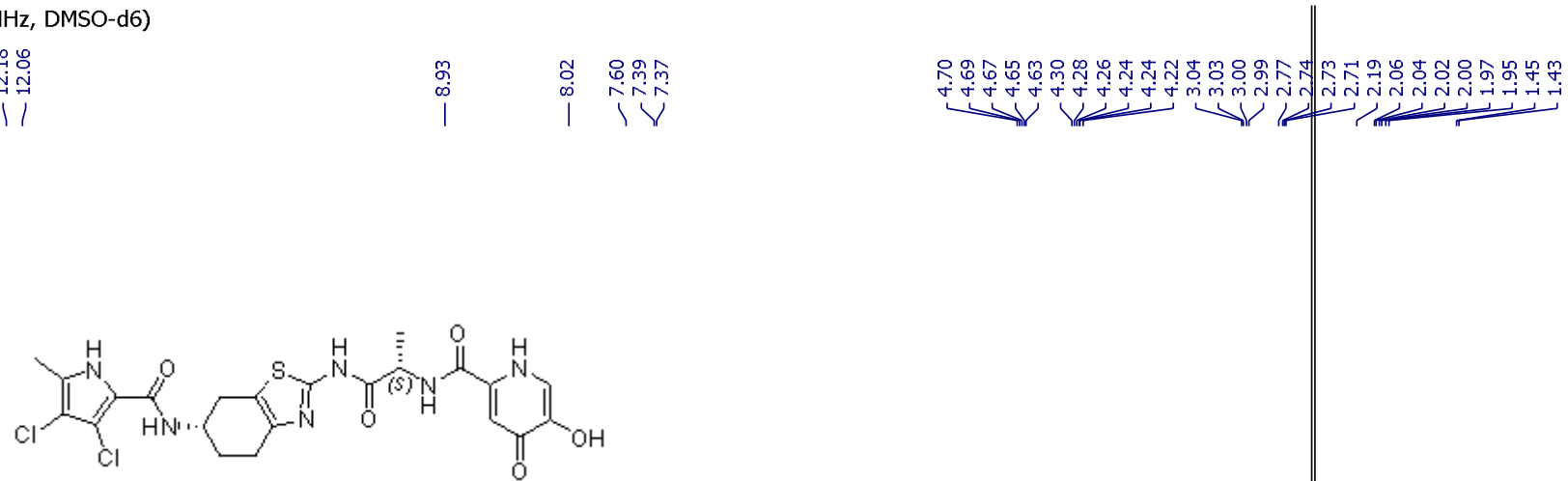

* ETOAC

\# water

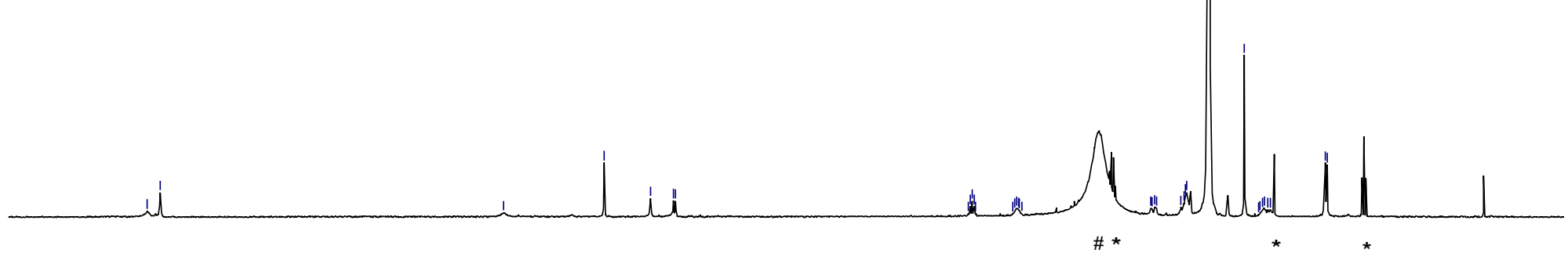

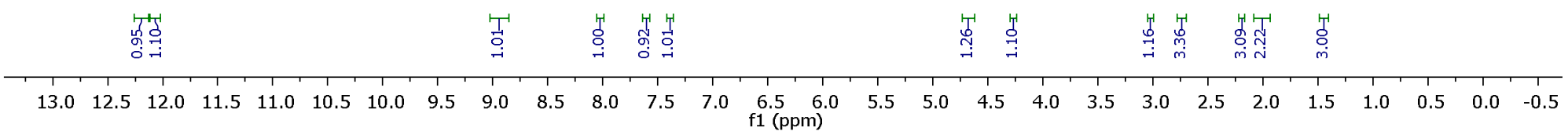


13C NMR (100 MHz, DMSO-d6)

l l l l l

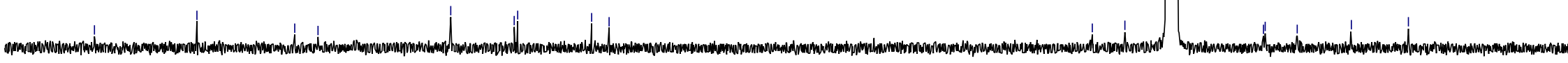

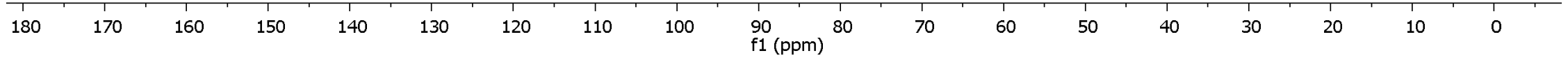


(S)-N-(3-((6-(3,4-Dichloro-5-methyl-1H-pyrrole-2-carboxamido)-4,5,6,7-tetrahydrobenzo[d]thiazol-2-yl)amino)-3-oxopropyl)-5-hydroxy-4-oxo-1,4dihydropyridine-2-carboxamide (47).

1H NMR (400 MHz, DMSO-d6)

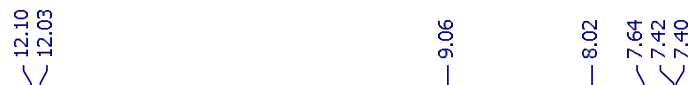

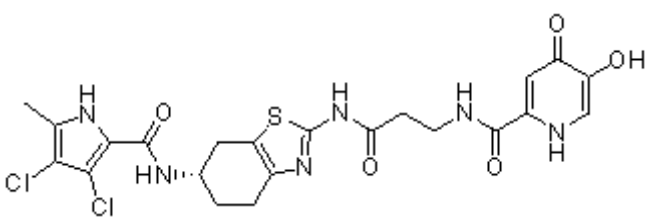

\# signal partially overlapped with water

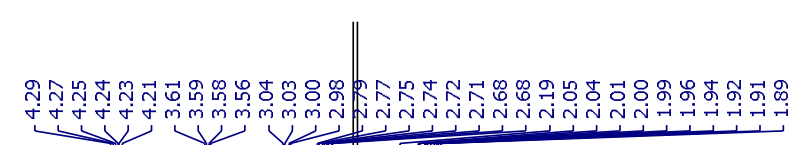

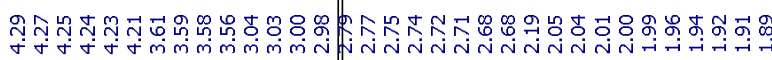
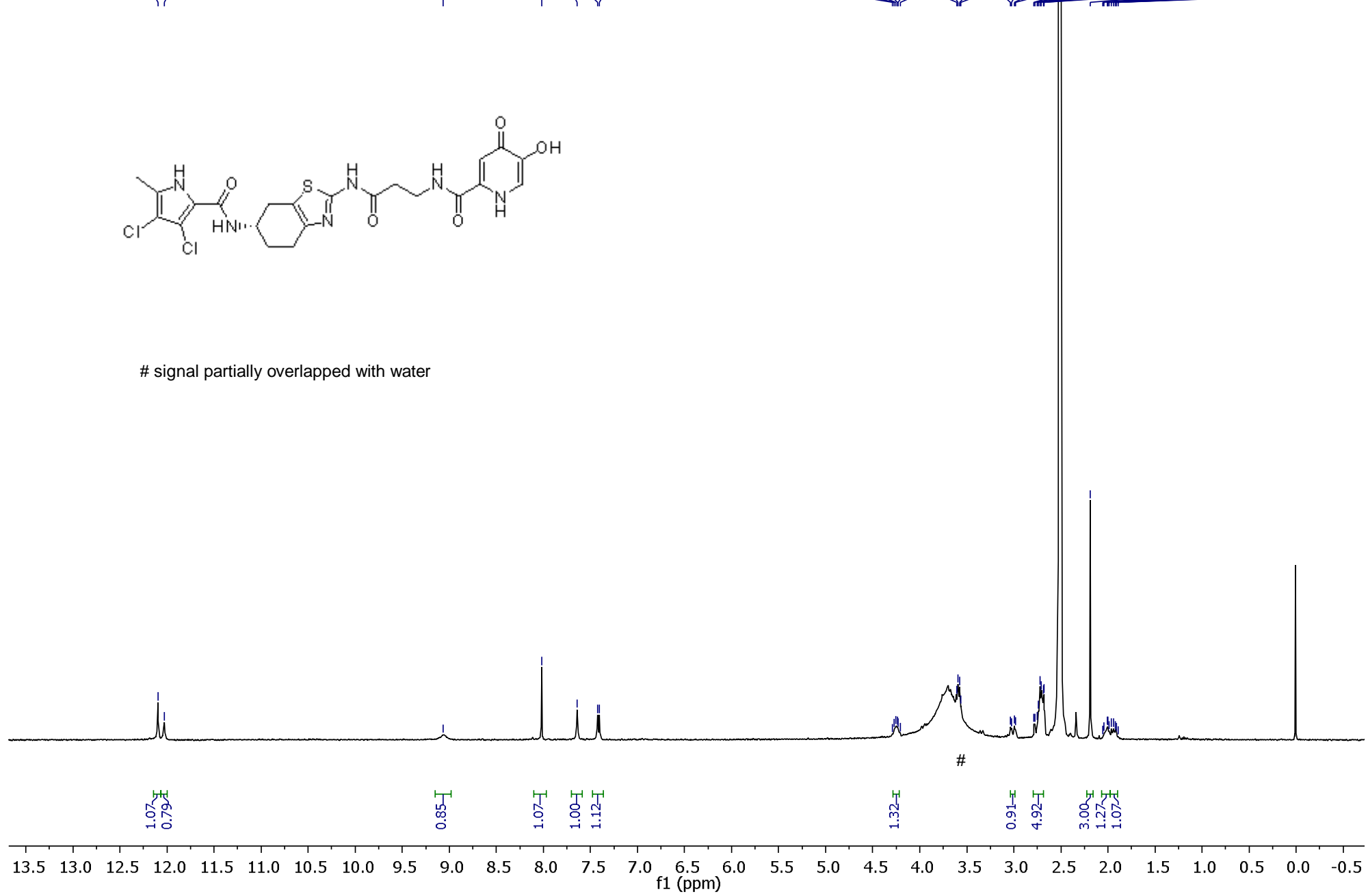
13C NMR (100 MHz, DMSO-d6)

$$
\text { l l }
$$

覃|
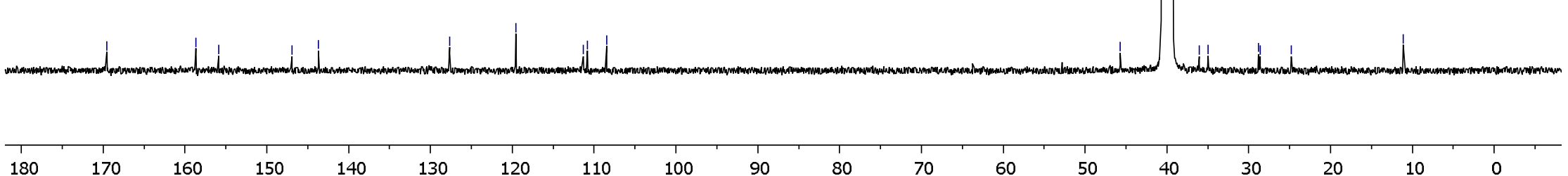

150
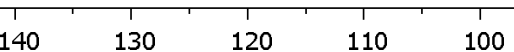

$90 \quad 80$
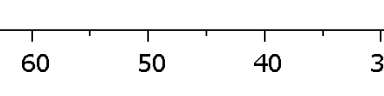

20

10 0 